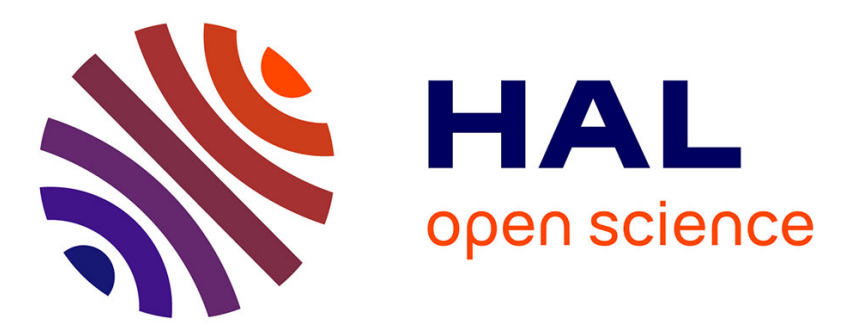

\title{
Consistent section-averaged shallow water equations with bottom friction
}

\author{
Victor Michel-Dansac, Pascal Noble, Jean-Paul Vila
}

\section{To cite this version:}

Victor Michel-Dansac, Pascal Noble, Jean-Paul Vila. Consistent section-averaged shallow water equations with bottom friction. European Journal of Mechanics - B/Fluids, 2021, 86, pp.123-149. hal01962186v3

\section{HAL Id: hal-01962186 \\ https://hal.science/hal-01962186v3}

Submitted on 13 Dec 2020

HAL is a multi-disciplinary open access archive for the deposit and dissemination of scientific research documents, whether they are published or not. The documents may come from teaching and research institutions in France or abroad, or from public or private research centers.
L'archive ouverte pluridisciplinaire HAL, est destinée au dépôt et à la diffusion de documents scientifiques de niveau recherche, publiés ou non, émanant des établissements d'enseignement et de recherche français ou étrangers, des laboratoires publics ou privés. 


\title{
Consistent section-averaged shallow water equations with bottom friction
}

\author{
Victor Michel-Dansac ${ }^{a, b, c}$, Pascal Noble ${ }^{a, b}$, Jean-Paul Vila ${ }^{a, b}$ \\ a Institut de Mathématiques de Toulouse, Université Toulouse 3 Paul Sabatier, 118 route de Narbonne, 31062 Toulouse Cedex 9, France \\ ${ }^{b}$ INSA Toulouse, 135 avenue de Rangueil, 31077 Toulouse Cedex 4, France \\ c [Present affiliation] Université de Strasbourg, CNRS, Inria, IRMA, F-67000 Strasbourg, France
}

\begin{abstract}
In this paper, we present a general framework to construct section-averaged models when the flow is constrained - e.g. by topography - to be almost one-dimensional. These models are consistent with the two-dimensional shallow water equations. After rewriting the two-dimensional shallow water equations in a suitable set of coordinates allowing to take care of a meandering configuration, we consider the quasi one-dimensional regime. Then, we expand the water elevation and velocity field in the spirit of the diffusive wave equations and establish a set of one-dimensional equations made of a mass, momentum and energy equations, which are close to the ones usually used in hydraulic engineering. Our model reduces to classical shallow water models with variable sections found in the literature. Out of these configurations, there is an $\mathcal{O}(1)$ deviation of our model from the classical ones. Finally, we present the main mathematical properties of our model and carry out numerical simulations to validate our approach by comparing the results to the full two-dimensional shallow water equations.
\end{abstract}

\section{Introduction}

In environmental modeling of free surface flows, the "shallow water" model is often used in order to reduce the complexity of the full Navier-Stokes equations and to reduce the computational cost implied by numerically solving such three-dimensional free surface flow equations. In order to derive the shallow water equations, it is assumed that the vertical variations of the water velocity are negligible with respect to the horizontal variations, and that the wavelengths of the phenomena of interest are much larger than the depth of the water. Under these assumptions (see for instance $[39,2,15]$ and references therein), it becomes natural to integrate the NavierStokes or Euler systems over the depth of the water, which naturally introduces the water height as a new variable, and eliminates the vertical component of the water velocity. The dispersive terms, which come from the vertical variations of the velocity, are usually neglected. Otherwise, they can be reintroduced as viscosity terms (see [15]) or approximated using an empirical or mathematical model (see for instance [14] and [2], Page 24, and references therein). Similar models may also be used for flows in pipes or ducts, see for instance $[22,35,18]$.

The shallow water model is commonly found in the simulation of various geophysical phenomena such as rivers, coastal flows or floods, and it is a standard model in hydraulics. The mathematical derivation of shallow water models is now well-documented for river flow simulations, under the assumption that the section of the channel is rectangular. For instance, see [31] and [4] in the context of respectively turbulent and laminar flows, and see [9] in the context of meander flows where the 2D shallow water equations are derived formally and numerical simulations are carried out under the assumption that sedimentation is neglected. See also [34] or [33] for, respectively, steady and unsteady flows in curved beds. 


\begin{tabular}{|c|c|c|c|}
\hline Notation & Meaning & Definition & Unit \\
\hline $\mathrm{h}$ & Water height & & $\mathrm{m}$ \\
\hline$u$ & Water velocity & $\mathbf{u}=\left(\mathbf{u}_{1}, \mathbf{u}_{2}\right)$ & $\mathrm{m} \mathrm{s}^{-1}$ \\
\hline$g$ & Gravity constant & & $\mathrm{ms}^{-2}$ \\
\hline$E_{2 D}$ & Energy in 2D & $\frac{h}{2}\|\mathbf{u}\|^{2}+g \frac{h^{2}}{2}$ & $m^{3} s^{-2}$ \\
\hline $\mathrm{b}_{0}$ & Longitudinal topography & & $\mathrm{m}$ \\
\hline$\phi$ & Transverse topography & & $\mathrm{m}$ \\
\hline Z & Topography & $\mathrm{Z}=\mathrm{b}_{0}+\phi$ & m \\
\hline $\mathrm{C}_{\mathrm{h}}$ & Chézy coefficient & & $m^{1 / 3} s^{-1}$ \\
\hline $\mathrm{C}$ & $2 \mathrm{D}$ friction model & $C=C_{h} h^{2 / 3}$ & $\mathrm{~m} \mathrm{~s}^{-1}$ \\
\hline$\xi$ & Local coordinates & $\xi=\left(\xi_{1}, \xi_{2}\right)$ & $\mathrm{m}$ \\
\hline$x$ & Global coordinates & $\mathbf{X}=\left(\mathrm{x}_{1}, \mathrm{x}_{2}\right)$ & $\mathrm{m}$ \\
\hline$\Xi_{-}, \Xi_{+}$ & Positions of the river banks & & $\mathrm{m}$ \\
\hline $\mathrm{R}$ & River curvature radius & & $\mathrm{m}$ \\
\hline$\sigma$ & Sign of the river curvature & & \\
\hline A & Matrix of the change of variables & & \\
\hline$|\mathcal{F}|$ & Determinant of $A^{-1}$ & $|\mathcal{F}|=1-\xi_{2} \frac{\sigma}{R}$ & \\
\hline$v$ & Local water velocity & $\boldsymbol{v}=\left(v_{1}, v_{2}\right)=A \boldsymbol{u}$ & $\mathrm{m} \mathrm{s}^{-1}$ \\
\hline$S$ & Wetted section & $S=\int_{\Xi_{-}}^{\Xi_{+}}|\mathcal{F}| \mathrm{h} \mathrm{d} \xi_{2}$ & $m^{2}$ \\
\hline $\mathrm{L}$ & River width & & $\mathrm{m}$ \\
\hline $\mathrm{H}$ & Average free surface & $\mathrm{S} \simeq \int_{0}^{\mathrm{H}} \mathrm{L}$ & $\mathrm{m}$ \\
\hline $\mathrm{Q}$ & Section-averaged discharge & $\mathrm{Q}=\int_{\Xi_{-}}^{\Xi_{+}}|\mathcal{F}| h v_{1} \mathrm{~d} \xi_{2}$ & $m^{3} s^{-1}$ \\
\hline$\Psi$ & Enstrophy & $\Psi=\frac{1}{S} \int_{\Xi_{-}}^{\Xi_{+}}|\mathcal{F}| \mathrm{h} \nu_{1}^{2} \mathrm{~d} \xi_{2}-\mathrm{U}^{2}$ & $m^{2} s^{-2}$ \\
\hline$\Pi$ & Potential & $\Pi=\frac{1}{\mathrm{su}} \int_{\Xi_{-}}^{\Xi_{+}}|\mathcal{F}| \mathrm{h} v_{1}^{3} \mathrm{~d} \xi_{2}-\mathrm{U}^{2}$ & $m^{2} s^{-2}$ \\
\hline$\beta$ & Boussinesq coefficient & $\beta=1+\frac{\psi}{\mathrm{u}^{2}}$ & \\
\hline$\alpha$ & Coriolis coefficient & $\alpha=1+\frac{\Pi}{u^{2}}$ & \\
\hline u & Longitudinal velocity scaling & & $\mathrm{m} \mathrm{s}^{-1}$ \\
\hline$\nu$ & Transverse velocity scaling & & $\mathrm{ms}^{-1}$ \\
\hline $\mathcal{H}$ & Water height scaling & & $\mathrm{m}$ \\
\hline$x$ & Longitudinal coordinates scaling & & $\mathrm{m}$ \\
\hline$y$ & Transverse coordinates scaling & & $\mathrm{m}$ \\
\hline $\mathcal{R}$ & Curvature radius scaling & & $\mathrm{m}$ \\
\hline $\mathcal{B}$ & Topography scaling & & $\mathrm{m}$ \\
\hline $\mathrm{e}_{0}$ & Friction scaling & & $\mathrm{ms}^{-1}$ \\
\hline $\mathrm{I}_{0}$ & Topography slope scaling & $\mathrm{I}_{0}=\mathcal{B} / x$ & \\
\hline $\mathrm{J}_{0}$ & Friction slope scaling & $\mathrm{J}_{0}=\mathrm{U}^{2} / \mathrm{e}_{0}^{2}$ & \\
\hline $\mathrm{R}_{v}$ & Velocity ratio & $\mathrm{R}_{v}=v / \mathrm{u}$ & \\
\hline $\mathrm{R}_{\mathrm{l}}$ & Length ratio & $R_{l}=y / x$ & \\
\hline$R_{y}$ & Curvature ratio & $R_{y}=y / \mathcal{R}$ & \\
\hline$\delta$ & Long wave parameter & $\delta=\mathcal{H} / x$ & \\
\hline $\mathrm{F}$ & Froude number & $\frac{1}{\mathrm{~F}^{2}}=\frac{\mathrm{g} \mathcal{H}}{\mathrm{U}^{2}}$ & \\
\hline$\varepsilon$ & Main scaling parameter & $\frac{\delta \mathrm{F}^{2}}{\mathrm{~J}_{0}}$ & \\
\hline$\Lambda$ & Corrected slope & $\Lambda=-\frac{I_{0}}{J_{0}} b_{0}^{\prime}-\frac{\delta}{J_{0}} H_{\xi_{1}}$ & \\
\hline
\end{tabular}


In this paper, we will focus on this particular regime of fixed topographies, which do not depend on time. The shallow water equations on a two-dimensional geometry usually read as follows:

$$
\left\{\begin{array}{l}
h_{t}+\nabla_{\mathbf{X}} \cdot(h \mathbf{u})=0, \\
\boldsymbol{u}_{\mathrm{t}}+\mathbf{u} \cdot \nabla_{\mathbf{X}} \mathbf{u}+g \boldsymbol{\nabla}_{\mathbf{X}} \mathbf{h}=g\left(-\nabla_{\mathbf{X}} \mathbf{Z}-\frac{\mathbf{u}\|\mathbf{u}\|}{\mathrm{C}(\boldsymbol{h}, \mathbf{X})^{2}}\right),
\end{array}\right.
$$

where $g$ is the gravity constant, $h$ the fluid height, $u=\left(u_{1}, u_{2}\right) \in \mathbb{R}^{2}$ the fluid velocity and $C(h, X)$ is a 2D friction model. The unknowns $h$ and $\boldsymbol{u}$ depend on the time variable $t$ and the space variable $\mathbf{X}=\left(x_{1}, x_{2}\right) \in \mathbb{R}^{2}$. For the sake of generality, we have chosen an arbitrary friction law. In some applications, a simple friction law, which only involves the water height, can be used by taking

$$
C(h, X)^{2}=C_{h}^{2} h^{p}
$$

where $C_{h}^{2}$ is the Chézy friction term and $p$ is usually taken equal to $4 / 3$. The dependency in $X$ of the friction model is supposed to come from the Chézy friction coefficient $C_{h}$.

More complex models, providing a more accurate description of physical phenomena, also exist in the literature. For instance, one may be referred to [17] for a multi-dimensional model of turbulent hydraulic jumps or to [28] for a model with dispersive and dissipative effects. However, in the present paper, we choose to consider the standard 2D shallow water system (1.1) for the sake of simplicity and to be able to perform the forthcoming mathematical developments.

In order to simplify the equations and reduce the computational efforts involved in solving the full twodimensional shallow water equations, we will consider the section-averaged shallow water equations. These section-averaged models are of particular interest in hydraulic engineering due to their simplicity and reduced computational cost. Such section-averaged models are particularly relevant to the specific geometry of a river (that is to say, a channel whose typical length is much greater than its typical width). However, deriving sectionaveraged models like (1.2) from two-dimensional models like (1.1) requires taking into account the specificities of the flow and of the geometry, usually through a relevant non-dimensionalization and subsequent asymptotic analysis. Indeed, the river is supposed to flow in the presence of meanders and the cross-section is variable.

The usual section-averaged models are obtained by integrating the 2D system (1.1) over the width of the channel, to get:

$$
\left\{\begin{array}{l}
S_{t}+Q_{x}=0, \\
Q_{t}+\left(\beta \frac{Q^{2}}{S}\right)_{x}+g S H_{x}=g S(\mathcal{J}-J),
\end{array}\right.
$$

where $S$ is the wetted section, $\mathrm{H}$ the average level of the water surface (i.e. the free surface), $\mathrm{Q}$ the sectionaveraged flow discharge, $\mathcal{J}$ is the main longitudinal slope of the river, $\mathcal{J}$ is a $1 \mathrm{D}$ friction model, and $\beta$ is the Boussinesq coefficient, defined below. The geometric quantities are displayed on figure 1.

A usual expression of the friction model $\mathcal{J}$ is the following one:

$$
\mathcal{J}=\frac{\mathrm{Q}|\mathrm{Q}|}{\mathrm{c}_{\mathrm{S} W}^{2}}
$$

where the friction coefficient $c_{S W}$ depends on $x$ and $h$. For instance, the Chézy formula prescribes

$$
c_{S W}^{2}=C_{1 D}^{2} R_{h} S^{2}
$$




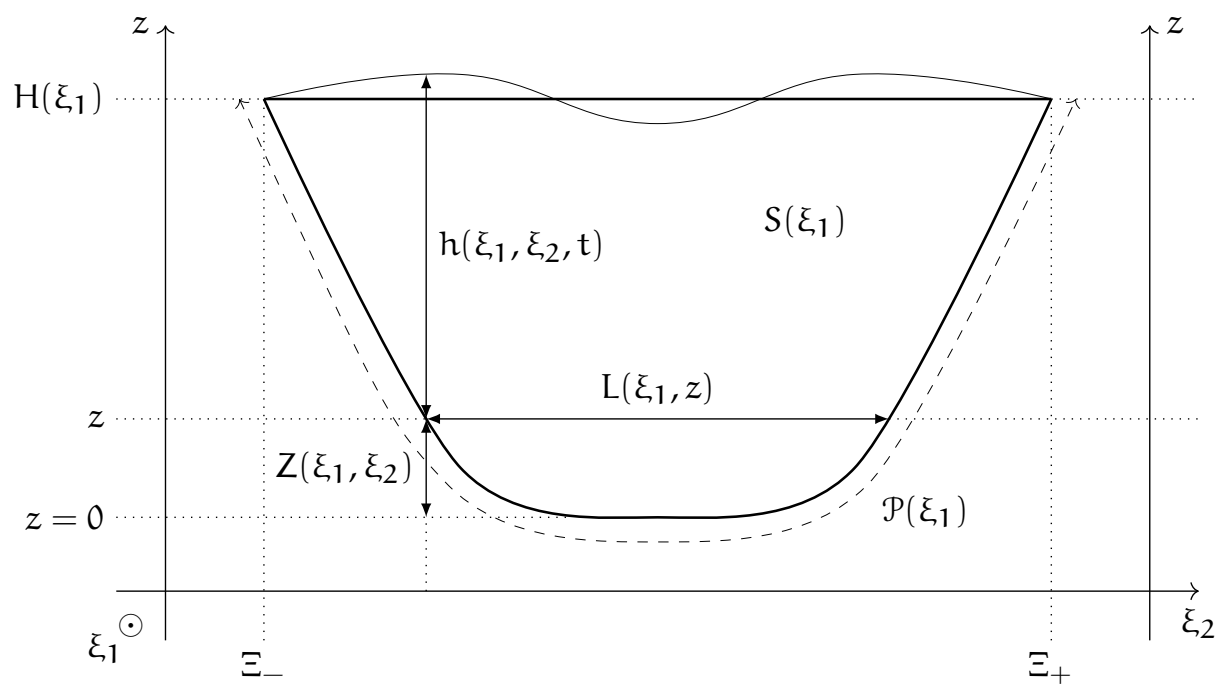

Figure 1: Sketch of a slice of the channel geometry and notations: $\xi_{1}$ and $\Xi_{-} \leqslant \xi_{2} \leqslant \Xi_{+}$are the longitudinal and transverse local coordinates, $z$ is the depth coordinate, $h$ is the water height, $\mathrm{H}$ is the average free surface position, $\mathrm{S}$ is the wetted section, $\mathcal{P}$ is the wetted perimeter, $\mathrm{L}$ is the function representing the width of the channel and $Z$ is the topography function.

where $C_{1 D}$ is a friction coefficient, where $R_{h}$ is the hydraulic radius, given by $R_{h}=S / \mathcal{P}$, and where $\mathcal{P}$ is the wetted perimeter, see figure 1 . An alternative is the Manning-Strickler formula

$$
c_{S W}^{2}=K_{s}^{2} R_{h}^{4 / 3} S^{2}
$$

where $K_{s}$ is the Manning-Strickler coefficient. Note that other formulas were derived in various situations, e.g. triangular, trapezoidal, circular or compound channels, to get complicated forms of the bottom friction valid mostly for stationary flows $[42,41]$. One could also consider the Reynolds number of the flow to derive friction laws based on the channel roughness, see for instance [25] for tabulated values or [31] for analytic expressions.

Here, $\beta \geqslant 1$ is the so-called Boussinesq term, which links the $1 \mathrm{D}$ behavior to the underlying $2 \mathrm{D}$ flow by accounting for the variations of the cross-section. Indeed, it is defined as follows:

$$
\beta=\frac{\int h u_{1}^{2} d y}{S u^{2}}
$$

where $\mathrm{U}=\mathrm{Q} / \mathrm{S}$ is the averaged $1 \mathrm{D}$ velocity and where $\mathrm{u}_{1}$ is the $2 \mathrm{D}$ velocity in the $\mathrm{x}$-direction, with the integral taken over the width of the channel. As soon as the flow is one-dimensional, i.e. constant in the cross-stream direction, $\beta$ is equal to 1. Many practical hydraulic engineering applications set $\beta$ to some constant value, close to 1 if the cross-section is smooth and somewhat larger if the cross-section becomes more complex (see for instance $[6,36])$. This means that, according to the table in [6] (Page 28), an error of up to $30 \%$ can be introduced in the discharge computation by setting a wrong value of $\beta$. In addition, modifying the discharge equation in (1.2) by adding the Boussinesq coefficient $\beta$ implies that the energy conservation is no longer satisfied. Indeed, one would also have to introduce the Coriolis coefficient $\alpha$ in the energy equation, defined similarly to the Boussinesq coefficient:

$$
\alpha=\frac{\int h u_{1}^{3} d y}{S u^{3}}
$$

This framework has been introduced almost two centuries ago in [7]; one of our goals in this paper is to suggest a better understanding of these issues. Indeed, a more involved discussion of these Boussinesq and Coriolis coefficients is available in section 5.1, starting with their precise definitions (5.2). 
It is our purpose to address the question of deriving $1 \mathrm{D}$ consistent shallow water models in the presence of meanders and for arbitrary geometries of the channel. To define the consistency of the model, a relevant small scaling parameter $\varepsilon \ll 1$ will be introduced. Contrary to the usual long wave approximation, in the present setting, this purely geometrical parameter $\varepsilon=\frac{\mathcal{C}^{2}}{\mathrm{~g} X}$ depends on the bottom friction scaling $\mathcal{C}$ and the typical wavelength $X$. Thanks to this parameter, we are able to compute asymptotic expansions of the free surface $H$ and the section-averaged discharge $\mathrm{Q}$, up to $\mathcal{O}\left(\varepsilon^{2}\right)$. On the one hand, the $1 \mathrm{D}$ model will be consistent up to the zerothorder if it introduces a $\mathcal{O}(\varepsilon)$ error on the free surface and the discharge. On the other hand, the 1D model will be consistent up to the first-order if a $\mathcal{O}\left(\varepsilon^{2}\right)$ error is introduced on the free surface and the discharge. In particular, we emphasize that the zeroth-order accuracy will be ensured by a new expression of the $1 \mathrm{D}$ friction coefficient. Usually, hydraulic engineering models are able to recover either free surface or the discharge up to $\mathcal{O}(\varepsilon)$ by using empirical friction coefficients. Getting an analytic expression for the 1D friction coefficient that recovers both free surface and discharge up to $\mathcal{O}(\varepsilon)$ is one of the new results of this manuscript.

\subsection{Structure of the paper}

In section 2, we introduce the 2D shallow water model, which we consider as the exact model for the flow of a meandering river. We write this system in a set of curvilinear coordinates in order to describe meandering. Then, in order to write a simplified 1D model, we introduce several scaling assumptions and perform a nondimensionalization of the 2D shallow water system, which is then averaged across the width of the channel.

Section 3 is dedicated to carrying out asymptotic expansions of the free surface and the velocity field in the onedimensional limit in order to close the section-averaged shallow water equations. Regarding the free surface, we show that it varies around a horizontal average value. Then, we exhibit expressions of the averaged discharge rate, which only depend on geometric terms and on the average free surface $\mathrm{H}$. They yield non-standard kinematic and diffusive wave approximations, which can depend on the slope of the free surface in addition to the topography slope, and which degenerate to the standard ones when the free surface slope vanishes. In addition, the influence of the meanders on the water surface is similar to the one obtained in studies involving the centrifugal force (see for instance [6], Page 447).

In section 4, in the strongly meandering case, we build a zeroth-order shallow water model: the friction term is built so as to ensure the consistency of the discharge rate to the main order. The structure is very simple, completely similar to the section-averaged shallow water models found in the literature (see e.g. [10]). The zeroth-order is achieved solely by manipulating the discharge equation.

In section 5, we propose our new first-order model and analyze its mathematical structure in the case of weakly meandering channels. It is a four-equation model describing the evolution of the wetted surface, the discharge rate, the energy and an additional quantity, called "enstrophy", which accounts for the vorticity of the flow in the cross-stream direction. This new model provides a first-order accurate description of the surface elevation and the discharge rate; in addition, it is hyperbolic. Contrary to the zeroth-order model, here, both zeroth-order and first-order approximations are obtained by introducing a suitable energy equation. In addition, the Boussinesq coefficient $\beta$ and the Coriolis coefficients $\alpha$ turn out to correspond to the new enstrophy and potential variables, respectively. As a consequence, this first-order model is able to overcome the shortcomings of the previous hydraulic engineering models, which usually set $\alpha$ and $\beta$ to some constant value.

The last section of this paper, section 6 , is dedicated to a numerical validation of our model. We compare the accuracy of three section-averaged models: the two (zeroth- and first-order) models we derived in this paper and a section-averaged shallow water model simply obtained by assuming that the downstream velocity is constant across the channel section (and which is obviously non consistent for channels that are not U-shaped).

Finally, in section 7, we summarize the results we have obtained in the previous sections, and we provide a short conclusion. 


\subsection{Perspectives}

In this paper, we have restricted our attention to fixed topographies. It is of particular interest to consider this type of model in the presence of sedimentation. In [26, 27], such a problem was considered for stationary flows in order to determine the mechanisms of migration of meanders; in [11], the author discusses the modification of the geometry incurred by sediment transport in curved channels. A model was formally derived and the study was completed with various numerical simulations. However, the asymptotic analysis was conducted with non consistent assumptions on the velocity profile. A non-stationary model with sedimentation was derived in [5] but for channels with vertical walls. We expect that our approach can be extended to transport of sediments in channels with arbitrary cross-sections in a similar way.

Another problem of interest is the coupling between 1D and 2D shallow water models. Here, the goal is either to carry out a complete modeling of an estuary, see for instance [1], or to model river floods, see for instance [12]. Our model is built directly from the 2D shallow water equations, which ensures a natural coupling between the two models.

As mentioned before, more complex phenomena are modeled by enhancing the 2D shallow water system (1.1) with additional equations (see $[17,28]$ ). It would be of particular interest to derive 1D models from these enhanced 2D systems, in order to account, for example, for dispersive and dissipative effects.

\section{Governing equations}

In this section, we consider the two-dimensional shallow water equations with bottom friction, governed by (1.1). This system admits an additional energy balance law:

$$
\left(E_{2 D}\right)_{t}+\nabla_{\mathbf{X}} \cdot\left(\mathbf{u}\left(E_{2 D}+\frac{1}{2} g h^{2}\right)\right)=g h\left(-\mathbf{u} \cdot \nabla_{\mathbf{X}} Z-\frac{\|\mathbf{u}\|^{3}}{C(h, X)^{2}}\right) .
$$

where the energy $E_{2 D}$ is given by $E_{2 D}=\frac{h}{2}\|\mathbf{u}\|^{2}+g \frac{h^{2}}{2}$. This equation can also be rewritten with a negative source term:

$$
\left(E_{2 D}+g h Z\right)_{t}+\nabla_{\mathbf{X}} \cdot\left(\mathbf{u}\left(E_{2 D}+g h Z+\frac{1}{2} g h^{2}\right)\right)=-g h \frac{\|\mathbf{u}\|^{3}}{C(h, X)^{2}} .
$$

We now write the system (1.1) in a reference frame which fits with the geometry of the channel. In order to study the limit of quasi one-dimensional flows, we write the new system in a dimensionless form together with its exact section-averaged version.

\subsection{Parameterization of the problem}

To deal with the meanders of the river, we introduce a parameterization of the river bed: for that purpose, we assume that it follows a parameterized curve $r: \xi_{1} \mapsto\left(\hat{x}\left(\xi_{1}\right), \hat{y}\left(\xi_{1}\right)\right)$ such that $\hat{x}^{\prime}\left(\xi_{1}\right)^{2}+\hat{y}^{\prime}\left(\xi_{1}\right)^{2}=1$, see figure 2. Since this relation is satisfied, we naturally introduce a function $\theta\left(\xi_{1}\right)$ such that $\hat{x}^{\prime}\left(\xi_{1}\right)=\cos \left(\theta\left(\xi_{1}\right)\right)$ and $\hat{y}^{\prime}\left(\xi_{1}\right)=\sin \left(\theta\left(\xi_{1}\right)\right)$.

We then naturally introduce the Frenet reference frame, defined by a vector $\mathbf{T}\left(\xi_{1}\right)$ tangent to the flow and a vector $\mathbf{N}\left(\xi_{1}\right)$ orthogonal to the flow. The tangent vector $\mathbf{T}\left(\xi_{1}\right)$ is defined as follows:

$$
\mathbf{T}\left(\xi_{1}\right):=\frac{\mathbf{r}^{\prime}\left(\xi_{1}\right)}{\left\|\mathbf{r}^{\prime}\left(\xi_{1}\right)\right\|}=\left(\cos \left(\theta\left(\xi_{1}\right)\right), \sin \left(\theta\left(\xi_{1}\right)\right)\right) .
$$

In the usual Frenet reference frame, the normal vector $\mathbf{N}\left(\xi_{1}\right)$ is chosen to be orthogonal to $\mathbf{T}\left(\xi_{1}\right)$ and pointing towards the center of the osculating circle of the curve $r$. This is not desirable in the context of a meandering river, 
since the orientation of the frame would sharply change with each meander. Instead, we define $\mathbf{N}\left(\xi_{1}\right)$ such that is always pointing towards the left of the river flow, as follows:

$$
\mathbf{N}\left(\xi_{1}\right):=\left(-\sin \left(\theta\left(\xi_{1}\right)\right), \cos \left(\theta\left(\xi_{1}\right)\right)\right)= \begin{cases}\sigma\left(\xi_{1}\right) \frac{\mathbf{T}^{\prime}\left(\xi_{1}\right)}{\left\|\mathbf{T}^{\prime}\left(\xi_{1}\right)\right\|} & \text { if } \sigma\left(\xi_{1}\right) \neq 0, \\ \mathbf{R}_{\frac{\pi}{2}} \mathbf{T}\left(\xi_{1}\right) & \text { otherwise, }\end{cases}
$$

where we have defined

$$
\sigma\left(\xi_{1}\right):=\operatorname{sgn}\left(\theta^{\prime}\left(\xi_{1}\right)\right)
$$

and where $\mathbf{R}_{\frac{\pi}{2}}$ is the matrix corresponding to the rotation of angle $\frac{\pi}{2}$. The quantity $\sigma\left(\xi_{1}\right)$ represents the orientation of the meander. Indeed, the river bed curves to the left if $\sigma\left(\xi_{1}\right)=1$, and it curves to the right if $\sigma\left(\xi_{1}\right)=-1$. Note that, contrary to the usual definition of $\mathbf{N}\left(\xi_{1}\right)$, the equations (2.1) and (2.2) also define the reference frame for a curve with vanishing curvature, i.e. when $\theta^{\prime}\left(\xi_{1}\right)=0$, see figure 2 . In this case,

We define the curvature radius $R\left(\xi_{1}\right)$ as follows:

$$
\mathbf{T}^{\prime}\left(\xi_{1}\right)=\left|\theta^{\prime}\left(\xi_{1}\right)\right| \mathbf{N}\left(\xi_{1}\right):=\frac{1}{R\left(\xi_{1}\right)} \mathbf{N}\left(\xi_{1}\right), \quad \text { i.e. } \quad R\left(\xi_{1}\right)=\frac{1}{\left|\theta^{\prime}\left(\xi_{1}\right)\right|} .
$$

Note that this curvature radius tends to infinity when $\theta^{\prime}\left(\xi_{1}\right)$ vanishes. Since this situation correspond to a straight river with no meanders, this behavior makes sense.

We now introduce the bijective change of variables from the Euclidean space to the Frenet reference frame. A point $\xi=\left(\xi_{1}, \xi_{2}\right)$ of the Frenet reference frame transforms into a point $X=\left(x_{1}, x_{2}\right)$ of the Euclidean space according to $\mathbf{X}\left(\xi_{1}, \xi_{2}\right)=\mathbf{r}\left(\xi_{1}\right)+\xi_{2} \mathbf{N}\left(\xi_{1}\right)$.

In what follows, we introduce the bijective change of variables $\xi=a(X)$ from the Euclidean space to the Frenet reference frame, whose inverse function is defined by

$$
a^{-1}\left(\xi_{1}, \xi_{2}\right)=\left(\begin{array}{l}
x_{1}\left(\xi_{1}, \xi_{2}\right) \\
x_{2}\left(\xi_{1}, \xi_{2}\right)
\end{array}\right)=\left(\begin{array}{l}
\hat{x}\left(\xi_{1}\right)-\xi_{2} \sin \left(\theta\left(\xi_{1}\right)\right) \\
\hat{y}\left(\xi_{1}\right)+\xi_{2} \cos \left(\theta\left(\xi_{1}\right)\right)
\end{array}\right) .
$$

In this reference frame, we assume that the bottom topography is parameterized by (see figure 1)

$$
Z\left(x_{1}\left(\xi_{1}, \xi_{2}\right), x_{2}\left(\xi_{1}, \xi_{2}\right)\right)=b_{0}\left(\xi_{1}\right)+\phi\left(\xi_{1}, \xi_{2}\right), \quad \phi\left(\xi_{1}, 0\right)=0, \quad \forall \xi_{1} \in \mathbb{R} .
$$

Therefore, the topography $Z$ is decomposed into two parts: a longitudinal part $b_{0}$, which records the main slope in the $\xi_{1}$-direction, i.e. in the main direction of the flow; and a transverse part $\phi$, which corresponds to the smaller topography variations in the cross-stream direction.

Now, we rewrite the shallow water equation system (1.1) in this new set of coordinates. For that purpose, we denote by

$$
A^{-1}\left(\xi_{1}, \xi_{2}\right)=\left(\begin{array}{lc}
\cos \left(\theta\left(\xi_{1}\right)\right)\left(1-\xi_{2} \frac{\sigma\left(\xi_{1}\right)}{R\left(\xi_{1}\right)}\right) & -\sin \left(\theta\left(\xi_{1}\right)\right) \\
\sin \left(\theta\left(\xi_{1}\right)\right)\left(1-\xi_{2} \frac{\sigma\left(\xi_{1}\right)}{R\left(\xi_{1}\right)}\right) & \cos \left(\theta\left(\xi_{1}\right)\right)
\end{array}\right)
$$

the Jacobian matrix of the change of variables $a^{-1}\left(\xi_{1}, \xi_{2}\right)$ defined above. Note that, if $\sigma\left(\xi_{1}\right)$ vanishes, i.e. if the curve becomes a straight line, this Jacobian matrix simply becomes the matrix describing a rotation of angle $\theta\left(\xi_{1}\right)$ with respect to the Euclidean frame. We also set

$$
|\mathfrak{F}|:=\operatorname{det}\left(A^{-1}\right)=1-\xi_{2} \frac{\sigma\left(\xi_{1}\right)}{R\left(\xi_{1}\right)} .
$$




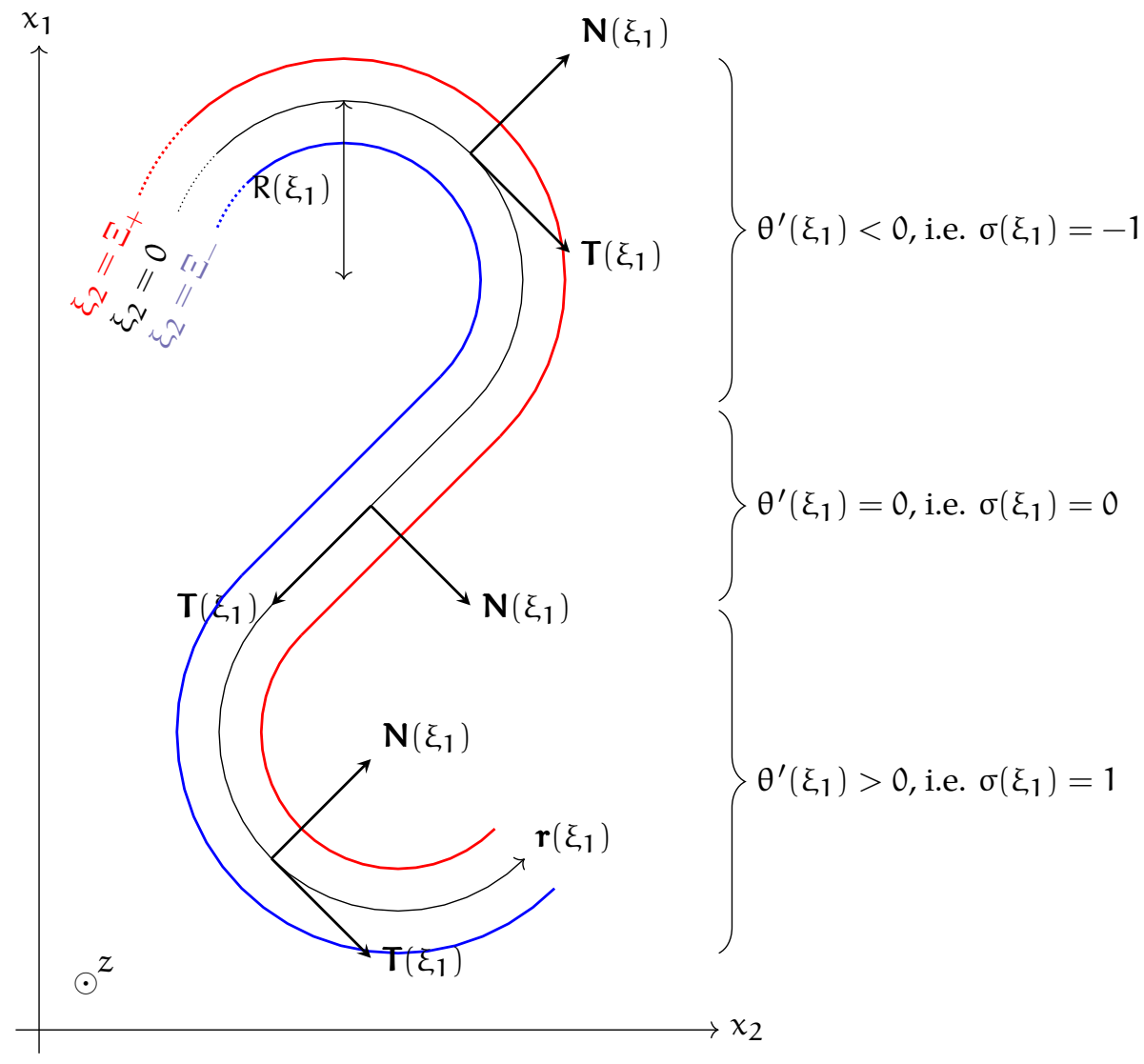

Figure 2: Sketch of a meandering river viewed from above. The Frenet reference frame $\left(\mathbf{T}\left(\xi_{1}\right), \mathbf{N}\left(\xi_{1}\right)\right)$ is displayed for several values of $\xi_{1}$. The left bank of the river is defined by $\xi_{2}=\Xi_{+}$, and the right bank is defined by $\xi_{2}=\Xi_{-}$. Note that the vector $\mathbf{N}\left(\xi_{1}\right)$ always points towards the left bank of the river. The right meander, straight section and left meander respectively correspond to $\theta^{\prime}\left(\xi_{1}\right)<0, \theta^{\prime}\left(\xi_{1}\right)=0$ and $\theta^{\prime}\left(\xi_{1}\right)>0$.

For the change of variables to be bijective, we need $|\mathcal{F}|>0$. This is valid as soon as $\left|\xi_{2}\right|<R\left(\xi_{1}\right)$. Assuming that $\left|\xi_{2}\right| / R\left(\xi_{1}\right)>|\mathcal{F}|_{m}$, we get $1-|\mathcal{F}|_{m}<|\mathcal{F}|<1+|\mathcal{F}|_{m}$. This inequality is satisfied in the usual context of a meandering river as represented by figure 2. More generally, the inequality imposes an upper bound on $\xi_{2}$, which may prevent us from considering a river with a strong lateral overflow, unless the geometry specifically takes this situation into account. However, in this case, the flow would become fully two-dimensional rather than one-dimensional, which would fall out of the scope of the current study.

We introduce the fluid velocity $\boldsymbol{v}=\left(v_{1}, v_{2}\right)$ in the Frenet frame, defined by $\boldsymbol{u}=A^{-1}\left(\xi_{1}, \xi_{2}\right) \boldsymbol{v}$. We denote by $A\left(x_{1}, x_{2}\right)$ the Jacobian matrix of the change of variables $a\left(x_{1}, x_{2}\right)$. After straightforward but tedious computations performed in Appendix A, the shallow water system reads, in the new reference frame:

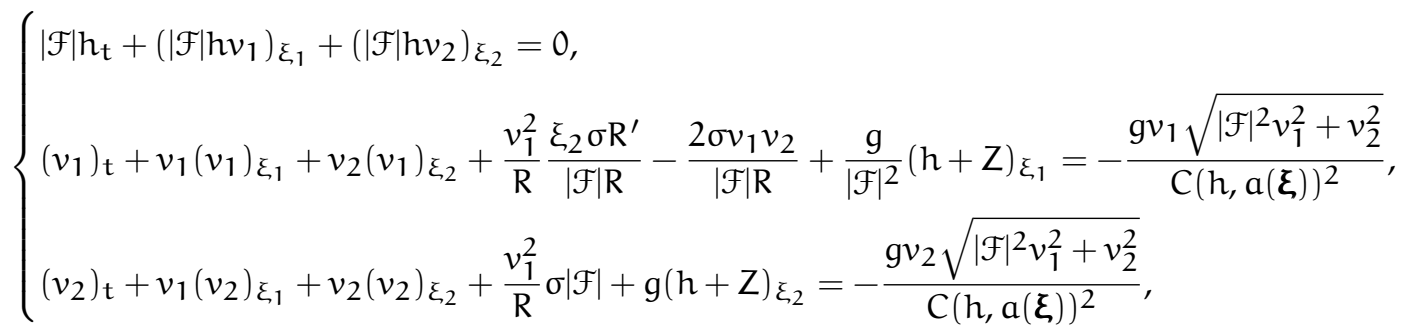

where the dependency in $\xi_{1}$ of $R\left(\xi_{1}\right)$ and $\sigma\left(\xi_{1}\right)$ is omitted. 
In that setting, the energy equation reads, for $E_{2 D}=\frac{h}{2}\|v\|^{2}+g \frac{h^{2}}{2}$ :

$$
\left(|\mathcal{F}| \mathrm{E}_{2 \mathrm{D}}\right)_{\mathrm{t}}+\nabla_{\boldsymbol{\xi}} \cdot\left(|\mathcal{F}| \boldsymbol{v}\left(\mathrm{E}_{2 \mathrm{D}}+\frac{1}{2} g \mathrm{~h}^{2}\right)\right)=g|\mathfrak{F}| \mathrm{h}\left(-\boldsymbol{v} \cdot \nabla \mathrm{Z}-\frac{\left\|A^{-1} \boldsymbol{v}\right\|^{3}}{\mathrm{C}(\mathrm{h}, \mathrm{a}(\boldsymbol{\xi}))^{2}}\right),
$$

or, equivalently, to highlight that the total energy $E_{2 D}+g h Z$ is only dissipated through friction:

$$
\left(|\mathcal{F}|\left(E_{2 D}+g h Z\right)\right)_{t}+\nabla_{\xi} \cdot\left(|\mathcal{F}| \boldsymbol{v}\left(E_{2 D}+g h Z+\frac{1}{2} g h^{2}\right)\right)=-g|\mathcal{F}| h \frac{\left\|A^{-1} \boldsymbol{v}\right\|^{3}}{C(h, a(\xi))^{2}} .
$$

\subsection{Dimensionless form of the system}

Next, in order to study the quasi one-dimensional flow limit, we write the system (2.3) in a dimensionless form. For that purpose, we introduce the following reference scales and rescaled quantities, denoted with a bar:

$$
v_{1}=: \mathcal{U} \overline{v_{1}}, \quad v_{2}=: \mathcal{V} \overline{v_{2}}, \quad h=: \mathcal{H} \bar{h}, \quad \xi_{1}=: X \overline{\xi_{1}}, \quad \xi_{2}=: y \overline{\xi_{2}},
$$

where $\mathcal{U}$ is the typical fluid velocity in the downstream direction whereas $\mathcal{V}$ is the typical fluid velocity in the cross-stream direction. The height $\mathcal{H}$ represents the typical fluid height, whereas $y$ is the typical channel width and $\mathcal{X}$ is a characteristic wavelength. We also introduce the typical time scale $\mathcal{T}=\mathcal{X} / \mathcal{U}$. The dimensionless mass conservation law then reads

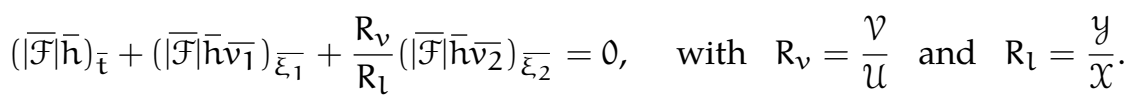

In what follows, we assume $R_{v}=R_{l}$ in order to keep the mass conservation law unchanged. We also take $R=: \mathcal{R} \bar{R}$, where $\mathcal{R}$ is the typical curvature radius found in the meanders of the river, and we set

$$
R_{y}=\frac{y}{\mathcal{R}} .
$$

Note that, with these notations, we get $\overline{R^{\prime}}=\frac{\mathcal{R}}{x} \bar{R}_{\overline{\xi_{1}}}$. In that setting, noting that $\sigma$ is already a dimensionless quantity, we have

$$
|\overline{\mathcal{F}}|=1-\mathrm{R}_{\mathrm{y}} \overline{\xi_{2}} \frac{\sigma\left(\overline{\xi_{1}}\right)}{\overline{\mathrm{R}}\left(\overline{\xi_{1}}\right)} .
$$

We scale the bottom topography as follows:

$$
\mathrm{b}_{\mathcal{O}}\left(\frac{\xi_{1}}{x}\right)=: \mathcal{B} \overline{\mathrm{b}_{0}}\left(\overline{\xi_{1}}\right), \quad \phi\left(\frac{\xi_{1}}{x}, \frac{\xi_{2}}{y}\right)=: \mathcal{H} \bar{\phi}\left(\overline{\xi_{1}}, \overline{\xi_{2}}\right) .
$$

Note that the scaling parameter of the transverse topography $\phi$ is $\mathcal{H}$, that is to say we take $\phi$ to be of the same order as the water height $h$. According to figure 1, this scaling is valid. Therefore, the rescaling of the topography reads:

$$
\mathrm{Z}\left(\frac{\xi_{1}}{x}, \frac{\xi_{2}}{y}\right)=: \mathcal{B} \overline{b_{0}}\left(\overline{\xi_{1}}\right)+\mathcal{H} \bar{\phi}\left(\overline{\xi_{1}}, \overline{\xi_{2}}\right)
$$

Note that the quantity $\bar{h}+\bar{\phi}$, viewed as the transverse topography added to the water height, represents the dimensionless free surface of the water flow.

Finally, we scale the bottom friction as follows:

$$
C\left(\frac{h}{\mathcal{H}}, \frac{\xi_{1}}{x}, \frac{\xi_{2}}{y}\right)=: \mathcal{C}_{0} \bar{C}\left(\bar{h}, \overline{\xi_{1}}, \overline{\xi_{2}}\right) .
$$


The dimensionless form of the shallow water system (2.3) therefore reads:

$$
\begin{aligned}
& \int(|\overline{\mathcal{F}}| \overline{\mathrm{h}})_{\overline{\mathrm{t}}}+\left(|\overline{\mathcal{F}}| \overline{\mathrm{h}} \overline{\nu_{1}}\right)_{\overline{\xi_{1}}}+\left(|\overline{\mathcal{F}}| \overline{\mathrm{h}} \overline{v_{2}}\right)_{\overline{\xi_{2}}}=0, \\
& \left\{\left(\overline{v_{1}}\right)_{\overline{\mathrm{t}}}+\overline{v_{1}}\left(\overline{v_{1}}\right)_{\overline{\xi_{1}}}+\overline{v_{2}}\left(\overline{v_{1}}\right)_{\overline{\xi_{2}}}+\frac{\mathrm{R}_{\mathrm{y}} \overline{v_{1}}}{|\overline{\mathcal{F}}| \overline{\mathrm{R}}} \sigma\left(\frac{\overline{\xi_{2}} \overline{\mathrm{R}^{\prime}}}{\overline{\mathrm{R}}} \overline{v_{1}}-2 \overline{v_{2}}\right)+\frac{1}{|\overline{\mathcal{F}}|^{2} \mathrm{~F}^{2}}(\overline{\mathrm{h}}+\bar{\phi})_{\overline{\xi_{1}}}\right. \\
& =\frac{-1}{\delta \mathrm{F}^{2}}\left(\frac{\mathrm{I}_{0} \overline{\bar{b}_{0}}}{|\overline{\mathcal{F}}|^{2}}+\mathrm{J}_{0} \frac{\overline{\overline{\mathrm{C}}_{1}}}{\overline{\mathrm{C}}^{2}} \sqrt{\overline{\mid \bar{F}}^{2}{\overline{v_{1}}}^{2}+\mathrm{R}_{\mathrm{l}}^{2}{\overline{v_{2}}}^{2}}\right), \\
& \left(\overline{v_{2}}\right)_{\overline{\mathrm{t}}}+\overline{v_{1}}\left(\overline{v_{2}}\right)_{\overline{\xi_{1}}}+\overline{v_{2}}\left(\overline{v_{2}}\right)_{\overline{\xi_{2}}}+\frac{\mathrm{R}_{\mathrm{y}}}{\mathrm{R}_{l}^{2}} \sigma \mid \overline{\mathcal{F} \mid} \frac{\overline{v_{1}}}{\overline{\mathrm{R}}}+\frac{1}{\mathrm{R}_{l}^{2} \mathrm{~F}^{2}}(\overline{\mathrm{h}}+\bar{\phi})_{\overline{\xi_{2}}}=-\frac{\mathrm{J}_{0}}{\delta \mathrm{F}^{2}} \overline{\overline{\mathrm{C}}^{2}} \sqrt{\overline{\mid \bar{F}}^{2}{\overline{v_{1}}}^{2}+\mathrm{R}_{\mathrm{l}}^{2}{\overline{v_{2}}}^{2}},
\end{aligned}
$$

where $\overline{\mathrm{R}}^{\prime}=\overline{\mathrm{R}}_{\overline{\xi_{1}}}$, where we have set the scaling parameters

$$
\mathrm{I}_{0}=\frac{\mathcal{B}}{X^{\prime}}, \quad \mathrm{J}_{0}=\frac{\mathcal{U}^{2}}{\mathcal{C}_{0}^{2}}, \quad \delta=\frac{\mathcal{H}}{X}, \quad \frac{1}{\mathrm{~F}^{2}}=\frac{\mathrm{g} \mathcal{H}}{\mathcal{U}^{2}},
$$

and where the dependency in $\bar{h}$ and $\bar{\xi}$ of the friction law $\bar{C}$ is omitted. The above dimensionless parameters correspond to the large-scale typical slope $I_{0}$, the friction slope $J_{0}$ (which we assume to be different from $I_{0}$ in order to handle very low large-scale slopes, present for instance in estuary configurations), the long wave parameter $\delta$ (which is small in the usual shallow water context), and the Froude number $F$. Note that the equation (2.4) has been written using only the dimensionless parameters $I_{0}, J_{0}, \delta, F, R_{l}$ and $R_{y}$. These six dimensionless parameters are independent, and they characterize the scales under consideration.

In order to highlight the variations of the free surface $\bar{h}+\bar{\phi}$ with respect to the transverse variable $\bar{\xi}_{2}$, we multiply (2.4c) by $R_{l}^{2} \mathrm{~F}^{2}$ and rearrange its terms. We get:

$$
(\overline{\mathrm{h}}+\bar{\phi})_{\overline{\xi_{2}}}+\mathrm{R}_{\mathrm{y}} \sigma|\overline{\mathcal{F}}| \mathrm{F}^{2} \frac{{\overline{v_{1}}}^{2}}{\overline{\mathrm{R}}}=-\frac{\mathrm{J}_{0} \mathrm{R}_{\mathrm{l}}^{2}}{\delta} \overline{\overline{\mathrm{C}_{2}}} \sqrt{\overline{\mathrm{C}}^{2}} \sqrt{|\overline{\mathcal{F}}|^{2}{\overline{v_{1}}}^{2}+\mathrm{R}_{\mathrm{l}}^{2}{\overline{v_{2}}}^{2}}-\mathrm{R}_{\mathrm{l}}^{2} \mathrm{~F}^{2}\left(\left(\overline{v_{2}}\right)_{\overline{\mathrm{t}}}+\overline{v_{1}}\left(\overline{v_{2}}\right)_{\overline{\xi_{1}}}+\overline{v_{2}}\left(\overline{v_{2}}\right)_{\overline{\xi_{2}}}\right) .
$$

The dimensionless form of the energy is $\overline{\mathrm{E}_{2 \mathrm{D}}}=\frac{\overline{\mathrm{h}}}{2}\left(\overline{\mid \mathcal{F}}^{2}{\overline{v_{1}}}^{2}+\mathrm{R}_{\mathrm{l}}^{2}{\overline{v_{2}}}^{2}\right)+\frac{\overline{\mathrm{h}}^{2}}{2 \mathrm{~F}^{2}}$, and the energy equation reads

$$
\left(|\overline{\mathcal{F}}| \overline{\mathrm{E}_{2 \mathrm{D}}}\right)_{\overline{\mathrm{t}}}+\nabla_{\bar{\xi}} \cdot\left(|\overline{\mathcal{F}}| \overline{\boldsymbol{v}}\left(\overline{\mathrm{E}_{2 \mathrm{D}}}+\frac{\overline{\mathrm{h}}^{2}}{2 \mathrm{~F}^{2}}\right)\right)=\frac{|\overline{\mathcal{F}}| \overline{\mathrm{h}}}{\delta \mathrm{F}^{2}}\left(-\mathrm{I}_{0} \overline{\mathrm{b}_{0}}{ }^{\prime} \overline{v_{1}}-\delta \overline{\boldsymbol{v}} \cdot \nabla_{\bar{\xi}} \bar{\phi}-\mathrm{J}_{0} \frac{\left(|\overline{\mathcal{F}}|^{2} \overline{\bar{v}}^{2}+\mathrm{R}_{\mathrm{l}}^{2}{\overline{v_{2}}}^{2}\right)^{3 / 2}}{\overline{\mathrm{C}}^{2}}\right),
$$

or, equivalently, to highlight that the dissipation of the total energy $\overline{E_{2 D}}+\frac{\bar{h}}{F^{2}}\left(\frac{I_{0}}{\delta} \overline{b_{0}}+\bar{\phi}\right)$ comes from the friction term:

$$
\begin{aligned}
\left(|\mathcal{F}|\left(\overline{\mathrm{E}_{2 \mathrm{D}}}+\frac{\overline{\mathrm{h}}}{\mathrm{F}^{2}}\left(\frac{\mathrm{I}_{0}}{\delta} \overline{\mathrm{b}_{\mathrm{O}}}+\bar{\phi}\right)\right)\right)_{\overline{\mathrm{t}}} & +\nabla_{\overline{\boldsymbol{\xi}}} \cdot\left(\overline{|\bar{F}|} \mid \overline{\boldsymbol{v}}\left(\overline{\mathrm{E}_{2 \mathrm{D}}}+\frac{\overline{\mathrm{h}}}{\mathrm{F}^{2}}\left(\frac{\mathrm{I}_{0}}{\delta} \overline{\mathrm{b}_{0}}+\bar{\phi}+\frac{\overline{\mathrm{h}}}{2}\right)\right)\right) \\
& =-\frac{\mathrm{J}_{0}}{\delta \mathrm{F}^{2}}|\overline{\mathcal{F}}| \overline{\mathrm{h}} \frac{\left(\left.\overline{\mid \mathcal{F}}\right|^{2}{\overline{v_{1}}}^{2}+\mathrm{R}_{\mathrm{l}}^{2}{\overline{v_{2}}}^{2}\right)^{3 / 2}}{\overline{\mathrm{C}}^{2}} .
\end{aligned}
$$

In what follows, we will not revert back to the dimensional quantities. For the sake of simplicity in the notations, from now on, the dimensionless quantities will be written without bars.

\subsection{Transverse averaging}

Recall that the ultimate goal of these developments is to provide a consistent 1D section-averaged model to approximate the full 2D system (2.4). To that end, we need to write an average of the 2D system over the width of the channel. In what follows, we denote by $\Xi_{-}\left(\xi_{1}, t\right)<\Xi_{+}\left(\xi_{1}, t\right)$ the positions of the edges of the river. Either 
the channel is walled, and the river sides are the same as the given channel sides, or the channel does not have walls, like in figure 1 . In this second case, $\Xi_{-}$and $\Xi_{+}$are defined implicitly as solutions of

$$
h\left(\xi_{1}, \Xi_{-}\left(\xi_{1}, t\right), t\right)=0, \quad h\left(\xi_{1}, \Xi_{+}\left(\xi_{1}, t\right), t\right)=0, \quad \forall t>0, \quad \forall \xi_{1} \in \mathbb{R} .
$$

These are smooth functions of $\xi_{1}$ and $t$ as long as $\phi \xi_{2}\left(\xi_{1}, \Xi_{ \pm}\left(\xi_{1}, t\right)\right) \neq 0$. This assumption is satisfied in the usual channels, for instance those with a trapezoidal or a triangular section. From now on, we consider this assumption to be satisfied. As a consequence, averaging the 2D system across the width of the channel consists in integrating the governing 2D equations between $\Xi_{-}$and $\Xi_{+}$. Note that it is possible to define the centerline of the channel such that $\Xi_{-}=-\Xi_{+}$. However, for the sake of generality, we do not make this assumption in the forthcoming developments.

We now apply this integration procedure to the equations (2.4a), (2.4b) (multiplied by $|\mathcal{F}| \mathrm{h})$ and (2.7), i.e. the mass, $x$-discharge and energy equations of the dimensionless $2 \mathrm{D}$ shallow water equations, to get:

$$
\left\{\begin{array}{l}
\mathrm{S}_{\mathrm{t}}+\mathrm{Q}_{\xi_{1}}=0, \\
\mathrm{Q}_{\mathrm{t}}+\left(\int_{\Xi_{-}}^{\Xi_{+}}|\mathcal{F}| \mathrm{h} v_{1}^{2} \mathrm{~d} \xi_{2}\right)_{\xi_{1}}+\frac{1}{\mathrm{~F}^{2}} \int_{\Xi_{-}}^{\Xi_{+}} \frac{\mathrm{h}}{|\mathcal{F}|}(\mathrm{h}+\phi)_{\xi_{1}} \mathrm{~d} \xi_{2}+\frac{\sigma \mathrm{R}_{\mathrm{y}}}{\mathrm{R}\left(\xi_{1}\right)} \int_{\Xi_{-}}^{\Xi_{+}} \mathrm{h} v_{1}\left(\xi_{2} v_{1} \frac{\mathrm{R}^{\prime}\left(\xi_{1}\right)}{\mathrm{R}\left(\xi_{1}\right)}-2 v_{2}\right) \mathrm{d} \xi_{2} \\
\quad=-\frac{\mathrm{I}_{0}}{\delta \mathrm{F}^{2}} \int_{\Xi_{-}}^{\Xi_{+}} \frac{\mathrm{h}}{|\mathcal{F}|} \mathrm{b}_{0}^{\prime} \mathrm{d} \xi_{2}-\frac{\mathrm{J}_{0}}{\delta \mathrm{F}^{2}} \int_{\Xi_{-}}^{\Xi_{+}} \frac{|\mathcal{F}| \mathrm{h} v_{1} \sqrt{|\mathcal{F}|^{2} v_{1}^{2}+\mathrm{R}_{\mathrm{l}}^{2} v_{2}^{2}}}{\mathrm{C}^{2}} \mathrm{~d} \xi_{2}, \\
\left(\int_{\Xi_{-}}^{\Xi_{+}}|\mathcal{F}| \mathrm{E}_{2 \mathrm{D}} \mathrm{d} \xi_{2}\right)_{\mathrm{t}}+\left(\int_{\Xi_{-}}^{\Xi_{+}}|\mathcal{F}| v_{1}\left(\mathrm{E}+\frac{\mathrm{h}^{2}}{2 \mathrm{~F}^{2}}\right) \mathrm{d} \xi_{2}\right)_{\xi_{1}} \\
=\frac{1}{\delta \mathrm{F}^{2}} \int_{\Xi_{-}}^{\Xi_{+}}|\mathcal{F}| \mathrm{h}\left(-\mathrm{I}_{0} \mathrm{~b}_{0}^{\prime} v_{1}-\delta \boldsymbol{v} \cdot \nabla_{\xi} \phi-\mathrm{J}_{0} \frac{\left(|\mathcal{F}|^{2} v_{1}^{2}+\mathrm{R}_{\mathrm{l}}^{2} v_{2}^{2}\right)^{3 / 2}}{\mathrm{C}^{2}}\right) \mathrm{d} \xi_{2},
\end{array}\right.
$$

where we have defined the wetted section $S$ and the average discharge $Q$ by:

$$
\mathrm{S}=\int_{\Xi_{-}}^{\Xi_{+}}|\mathcal{F}| \mathrm{hd} \xi_{2} \quad \text { and } \quad \mathrm{Q}=\int_{\Xi_{-}}^{\Xi_{+}}|\mathcal{F}| \mathrm{h} v_{1} \mathrm{~d} \xi_{2}
$$

\section{Asymptotic expansions}

We have exhibited a natural parameter in the system, which we denote by

$$
\varepsilon:=\frac{\delta \mathrm{F}^{2}}{\mathrm{~J}_{0}} .
$$

Recall the definitions of $\delta, \mathrm{F}^{2}$ and $\mathrm{J}_{0}$, given by (2.5) with respect to the scaling parameters. Then, the definition (3.1) of $\varepsilon$ reduces to

$$
\varepsilon=\frac{\mathrm{e}_{0}^{2}}{g x}
$$

Note that $\mathcal{C}_{0}^{2} / \mathrm{g}$ has the dimension of a length. Therefore, the parameter $\varepsilon$ is nothing but a geometrical term, in that it only depends on the permanent characteristics of the river rather than on the flow, linking the friction scaling with the typical wavelength.

In this section, we expand the fluid velocity field, in the case where $\varepsilon \ll 1, F^{2} \leqslant \mathcal{O}(1), I_{0} \leqslant \mathcal{O}(1), \delta / J_{0} \leqslant \mathcal{O}(1)$, for $R_{v}=R_{l}$, and in the quasi-dimensional setting $R_{l}=\mathcal{O}(\varepsilon)$. Note that the condition $R_{l}=\mathcal{O}(\varepsilon)$ is a geometric 
hypothesis, and it is satisfied in the regimes under consideration. We first consider the transverse equilibrium and show that, asymptotically, the free surface is horizontal. Then, we consider the zeroth-order longitudinal equilibrium where the flow is dominated by the competition between the gravity effects and the bottom friction, and for arbitrary meanders, i.e. $R_{y} \leqslant \mathcal{O}(1)$. Afterwards, we go a step further in the case where $R_{y}=\mathcal{O}(\varepsilon)$ to exhibit the complete first-order asymptotic regime. Finally, we compute the first-order sur-elevation of the free surface for a meandering river.

We can justify the regime $\varepsilon \ll 1$ as follows. Usually, the friction scaling $\mathcal{C}_{0}^{2}$ will be defined according to the Manning-Strickler law, to get $\mathcal{C}_{0}^{2}=\mathrm{K}_{\mathrm{s}}^{2} \mathcal{H}^{4 / 3}$. For standard values of $\mathrm{K}_{\mathrm{s}}$ around $30 \mathrm{~m}^{1 / 3} \cdot \mathrm{s}^{-1}, \mathcal{H}$ around $2 \mathrm{~m}$ and $\mathrm{g}$ around $10 \mathrm{~m} \cdot \mathrm{s}^{-2}$, we show that $\varepsilon \simeq \frac{100 \mathcal{H}}{x}=100 \delta$. Therefore, in the configurations of interest, the parameter $\varepsilon$ will be considered small as soon as the typical wavelength $X$ is large enough with respect to roughly $100 \mathcal{H}$. Moreover, according to the definition (3.1) of $\varepsilon$, we get $\varepsilon \simeq 100 \delta$ is equivalent to $F^{2} \simeq 100 \mathrm{~J} 0$, which means that $F^{2}$ should be small enough with respect to roughly $100 \mathrm{~J}_{0}$ for $\varepsilon$ to be small. Furthermore, we will consider the geometric hypothesis $R_{l}=\mathcal{O}(\varepsilon)$, which here means that $y$ should be small enough with respect to $100 \mathcal{H}$ for $\varepsilon$ to be small.

In addition, to consider gravity effects coming from both the slope of the topography and the slope of the free surface, we impose $\frac{I_{0}}{J_{0}}=\mathcal{O}(1)$ and $\frac{\delta}{J_{0}}=\mathcal{O}(1)$. This relationship is detailed further in section 3.1.2. This means that the friction slope should not be much smaller than the topography slope. In addition, in the specific case where $\mathrm{I}_{\mathcal{O}} \neq 0$, this also means that $\frac{\delta}{\mathrm{I}_{\mathcal{O}}}=\mathcal{O}(1)$, which is yet another purely geometric condition, rewritable as $\frac{\mathcal{H}}{\mathcal{B}}=\mathcal{O}(1)$.

The main goal of this section is to provide asymptotic expansions of the quantities that will be used in the forthcoming 1D models. Therefore, we exhibit expansions of the free surface $h+\phi$ and of the average discharge $Q$ with respect to the scaling parameters. In this section, we prove that

$$
\begin{aligned}
\mathrm{Q}\left(\xi_{1}, \mathrm{t}\right) & =\mathrm{Q}^{(\mathcal{O})}\left(\xi_{1}, \mathrm{t}\right)+\varepsilon \mathrm{Q}^{(1)}\left(\xi_{1}, \mathrm{t}\right)+\mathcal{O}\left(\varepsilon^{2}\right), \\
(\mathrm{h}+\phi)\left(\xi_{1}, \xi_{2}, \mathrm{t}\right) & =\mathrm{H}\left(\xi_{1}, \mathrm{t}\right)+\mathrm{F}^{2} \Delta \mathrm{H}\left(\xi_{1}, \xi_{2}, \mathrm{t}\right)+\mathcal{O}\left(\varepsilon \mathrm{F}^{2}\right),
\end{aligned}
$$

and we exhibit explicit expressions for $\mathrm{Q}^{(0)}, \mathrm{Q}^{(1)}$ and $\Delta \mathrm{H}$.

Note that $Q^{(0)}$ and $Q^{(0)}+\varepsilon Q^{(1)}$ respectively give models of kinematic and diffusive waves, respectively defined by $S_{t}+Q_{\xi_{1}}^{(0)}=0$ and $S_{t}+\left(Q^{(0)}+\varepsilon Q^{(1)}\right)_{\xi_{1}}=0$. These models will be nonstandard. Indeed, the standard models are given by

$$
\begin{aligned}
S_{t}+\left(Q_{S W}^{(0)} \xi_{\xi_{1}}=0\right. & \text { for the kinematic waves model, } \\
S_{t}+\left(Q_{S W}^{(0)}+\varepsilon Q_{S W}^{(1)}\right)_{\xi_{1}}=0 & \text { for the diffusive waves model, }
\end{aligned}
$$

where $Q_{S W}^{(0)}$ and $Q_{S W}^{(1)}$ are the zeroth- and first-order averaged discharge rates for the classical 1D shallow water system (1.2) with $\beta=1$. To obtain them, we first write (1.2) in dimensionless form, using the scaling developed in section 2.2, and with $\beta=1$. More details are given, for instance, in [20,23]. Then, we plug the asymptotic expansion $Q=Q_{S W}^{(0)}+\varepsilon Q_{S W}^{(1)}$ into this system. The balance between longitudinal slope and friction gives $Q_{S W}^{(0)}$, whereas $\mathrm{Q}_{\mathrm{SW}}^{(1)}$ is obtained by considering the left-hand side of the discharge equation:

$$
\mathrm{Q}_{\mathrm{SW}}^{(0)}=\operatorname{sgn}(\mathcal{J}) \sqrt{|\mathcal{J}|} \mathrm{c}_{\mathrm{SW}} \quad \text { and } \quad \mathrm{Q}_{\mathrm{SW}}^{(1)}=-\frac{\mathrm{Q}_{\mathrm{SW}}^{(0)}}{2 \mathrm{SJ}}\left(\left(\mathrm{Q}_{\mathrm{SW}}^{(0)}\right)_{\mathrm{t}}+\left(\frac{\left(\mathrm{Q}_{\mathrm{SW}}^{(0)}\right)^{2}}{\mathrm{~S}}\right)_{x}+\frac{\mathrm{SH}_{\mathrm{x}}}{\mathrm{F}^{2}}\right)
$$

\subsection{Zeroth-order expansions}

In this first part of section 3 , we consider a river with arbitrary meanders, i.e. $R_{y} \leqslant \mathcal{O}(1)$. The goal is to exhibit the zeroth-order asymptotic expansion in $\varepsilon$ satisfied by the unknowns of the system. 


\subsubsection{Transverse equilibrium}

In the asymptotic regime under consideration, the equation (2.6) for the free surface elevation reads

$$
\frac{\delta}{J_{0}}(h+\phi)_{\xi_{2}}=\mathcal{O}\left(\frac{\delta F^{2}}{J_{0}} R_{y}+R_{l}^{2}\left(1+\frac{\delta F^{2}}{J_{0}}\right)\right)=\mathcal{O}\left(\frac{\delta F^{2}}{J_{0}} R_{y}+R_{l}^{2}\right)
$$

In this framework, there exists a new unknown function $H\left(\xi_{1}, t\right)$, which corresponds to an average free surface, such that

noting that

$$
\frac{\delta}{J_{0}}\left(h\left(\xi_{1}, \xi_{2}, t\right)+\phi\left(\xi_{1}, \xi_{2}\right)\right)=\frac{\delta}{J_{0}} H\left(\xi_{1}, t\right)+\mathcal{O}\left(\frac{\delta F^{2}}{J_{0}} R_{y}+R_{l}^{2}\right)
$$

$$
\frac{\delta F^{2}}{J_{0}} R_{y}+R_{l}^{2}=\mathcal{O}(\varepsilon) \ll 1 .
$$

Thus, the asymptotic expansion (3.3) means that the free surface $h+\phi$ is flat (that is to say, it does not depend on $\left.\xi_{2}\right)$ up to $\mathcal{O}\left(F^{2} R_{y}\right)$. Furthermore, note that the expression (2.10) of the section $S$ offers a natural relationship between $S$ and $H$. Indeed, (2.10) yields

$$
S=\int_{\Xi_{-}}^{\Xi_{+}}|\mathcal{F}| \mathrm{hd} \xi_{2}=\int_{\Xi_{-}}^{\Xi_{+}}|\mathcal{F}|(\mathrm{H}-\phi) \mathrm{d} \xi_{2}+\mathcal{O}\left(\mathrm{F}^{2} \mathrm{R}_{\mathrm{y}}+\frac{\mathrm{J}_{0}}{\delta} \mathrm{R}_{\mathrm{l}}^{2}\right) .
$$

Therefore, up to $\mathcal{O}\left(F^{2} R_{y}+\frac{J_{0}}{\delta} R_{l}^{2}\right)$, knowing $H$ naturally gives a value of $S$, and reciprocally, knowing $S$ allows one to find a value of $\mathrm{H}$ at the cost of solving a nonlinear equation.

\subsubsection{Longitudinal equilibrium}

Let us now carry out an asymptotic expansion of the fluid velocity field $\left(v_{1}, v_{2}\right)$. The longitudinal discharge equation $(2.4 \mathrm{~b})$ can be written as

$$
\begin{aligned}
\frac{\delta}{J_{0}} \frac{(h+\phi) \xi_{1}}{|\mathcal{F}|^{2}}+\frac{I_{0}}{J_{0}} \frac{b_{0}^{\prime}}{|\mathcal{F}|^{2}} & +\frac{v_{1} \sqrt{|\mathcal{F}|^{2} v_{1}^{2}+R_{l}^{2} v_{2}^{2}}}{C^{2}} \\
& =-\frac{\delta F^{2}}{J_{0}}\left(\left(v_{1}\right)_{t}+v_{1}\left(v_{1}\right)_{\xi_{1}}+v_{2}\left(v_{1}\right)_{\xi_{2}}+\frac{R_{y} v_{1}}{|\mathcal{F}| \mathrm{R}}\left(\frac{\xi_{2} R^{\prime}}{R} v_{1}-2 \sigma v_{2}\right)\right)
\end{aligned}
$$

For the sake of clarity, we introduce the notation

$$
\tilde{\varepsilon}=\frac{\delta F^{2}}{J_{0}}\left(1+R_{y}\right)+R_{l}^{2}=\mathcal{O}(\varepsilon) \ll 1 .
$$

Recalling the definition (3.3) of the average free surface $H$, the equation (3.5) reads, in the asymptotic regime under consideration, as follows:

$$
\frac{1}{|\mathcal{F}|^{2}}\left(\frac{\mathrm{I}_{0}}{\mathrm{~J}_{0}} \mathrm{~b}_{0}^{\prime}+\frac{\delta}{\mathrm{J}_{0}} \mathrm{H}_{\xi_{1}}\right)+\frac{|\mathcal{F}| \nu_{1}\left|v_{1}\right|}{\mathrm{C}^{2}}=\mathcal{O}(\tilde{\varepsilon})
$$

The equation (3.6) is nothing but the definition of a uniform flow (see [6], Chapter 5), where the topography and the friction are balanced. Assuming the asymptotic expansion

$$
v_{1}\left(\xi_{1}, \xi_{2}, t\right)=v_{1}^{(0)}\left(\xi_{1}, \xi_{2}, t\right)+\mathcal{O}(\tilde{\varepsilon})
$$

on the longitudinal velocity, the equation (3.6) can be solved, to get

$$
v_{1}^{(0)}\left(\xi_{1}, \xi_{2}, t\right)=\frac{\Lambda\left(\xi_{1}, t\right)}{\sqrt{\left|\Lambda\left(\xi_{1}, t\right)\right|}} \frac{C\left(\xi_{1}, \xi_{2}, H\left(\xi_{1}, t\right)-\phi\left(\xi_{1}, \xi_{2}\right)\right)}{|\mathcal{F}|\left(\xi_{1}, \xi_{2}\right)^{3 / 2}},
$$


where we have defined the corrected slope

$$
\Lambda\left(\xi_{1}, t\right)=-\frac{I_{0}}{J_{0}} b_{0}^{\prime}\left(\xi_{1}\right)-\frac{\delta}{J_{0}} H_{\xi_{1}}\left(\xi_{1}, t\right) .
$$

The corrected slope $\Lambda$, rather than the main longitudinal slope $b_{0}^{\prime}\left(\xi_{1}\right)$, is involved in all asymptotic expansions. The global gravity effects, both stemming from the slope of the topography and the slope of the free surface, are merged in $\Lambda$. Let us remark that the choice $\mathrm{I}_{0} \neq \mathrm{J}_{0}$, here, allows us to consider a flow driven mainly by the free surface slope in the case of a channel with a very weak slope (a situation encountered for instance in an estuary). Note that we need $\Lambda=\mathcal{O}(1)$, i.e. to make sure that $\Lambda$ does not contain any term in $\mathcal{O}(1 / \varepsilon)$, for the asymptotic expansion $v_{1}^{(0)}$ to be well-defined. Since $\frac{I_{0}}{J_{0}}=\mathcal{O}(1)$ and $\frac{\delta}{J_{0}}=\mathcal{O}(1)$, we indeed observe that $\Lambda=\mathcal{O}(1)$, according to the definition (3.8) of $\Lambda$.

From this expansion, we can deduce an expansion of the transverse velocity $v_{2}$ by writing $v_{2}=v_{2}^{(0)}+\mathcal{O}(\tilde{\varepsilon})$ and using the mass conservation law:

$$
\left(|\mathcal{F}| \mathrm{h} v_{2}^{(0)}\right)_{\xi_{2}}=-|\mathcal{F}| h_{t}-\left(|\mathcal{F}| \mathrm{h} v_{1}^{(0)}\right)_{\xi_{1}}+\mathcal{O}(\tilde{\varepsilon}) .
$$

Then, we get

$$
|\mathcal{F}| h v_{2}^{(0)}\left(\xi_{1}, \xi_{2}, \mathrm{t}\right)=-\int_{\Xi_{-}\left(\xi_{1}, \mathrm{t}\right)}^{\xi_{2}}\left(|\mathcal{F}| h_{\mathrm{t}}+\left(|\mathcal{F}| \mathrm{h} v_{1}^{(0)}\right)_{\xi_{1}}\right)\left(\xi_{1}, \eta, t\right) d \eta+\mathcal{O}(\tilde{\varepsilon}) .
$$

Finally, note that the width-integrated longitudinal discharge $Q$ also satisfies $Q=Q^{(0)}+\mathcal{O}(\tilde{\varepsilon})$. Arguing the definition (3.7) of $v_{1}^{(0)}$, we get the following expansion:

$$
\mathrm{Q}^{(0)}:=\int_{\Xi_{-}}^{\Xi_{+}}|\mathcal{F}| \mathrm{h} v_{1}^{(0)} \mathrm{d} \xi_{2}=\int_{\Xi_{-}}^{\Xi_{+}} \sqrt{|\Lambda|} \operatorname{sgn}(\Lambda) \frac{|\mathcal{F}| \mathrm{hC}}{|\mathcal{F}| \sqrt{|\mathfrak{F}|}} \mathrm{d} \xi_{2} .
$$

Since $\Lambda$ does not depend on $\xi_{2}$, the definition of $\mathrm{Q}^{(0)}$ can be simplified as follows:

$$
\mathrm{Q}^{(0)}=\sqrt{|\Lambda|} \operatorname{sgn}(\Lambda) \int_{\Xi_{-}}^{\Xi_{+}} \frac{h \mathrm{C}}{\sqrt{|\mathfrak{F}|}} \mathrm{d} \xi_{2}
$$

Note that $\mathrm{Q}^{(0)}$ depends only on geometric quantities and on $\mathrm{H}$. Since $\mathrm{Q}^{(0)}$ comes from the definition of $v_{1}^{(0)}$, which stems for the definition (3.6) of a uniform flow, it turns out that $\mathrm{Q}^{(0)}$ represents the averaged discharge of a uniform flow. The expression of $\mathrm{Q}^{(0)}$ gives the discharge of a uniform flow, and it will therefore be used in the definition of the averaged friction term in the model developed in the next section. Moreover, the kinematic waves model $S_{t}+Q_{\xi_{1}}^{(0)}=0$ turns out to be nonstandard as soon as $H_{\xi_{1}} \neq 0$. Indeed, in usual kinematic waves models, $Q^{(0)}$ given by (3.2) depends on $H$, see for instance [20]. Here, because of $\Lambda, Q^{(0)}$ depends on both $H$ and $\mathrm{H}_{\xi_{1}}$. Since we consider that the effects of the free surface slope are relevant, it makes sense that these effects appear in the kinematic wave approximation.

\subsection{First-order expansion}

So far, we have provided a zeroth-order expansion of the unknowns of the problem, with respect to $\frac{\delta F^{2}}{J_{0}} R_{y}+R_{l}^{2}$ and $\tilde{\varepsilon}$. Let us now consider the next order. If we consider the general case $R_{y}=\mathcal{O}(1)$, going further to a firstorder expansion of the longitudinal velocity would be troublesome since, in this case, $\frac{\delta F^{2}}{J_{0}} R_{y}+R_{l}^{2}=\mathcal{O}(\varepsilon)$. As a result, we would get a term in $\varepsilon R_{y}$ in the first-order expansion of $H$, which would make the computations barely tractable.

Therefore, we first consider the case $R_{y}=\mathcal{O}(\varepsilon)$, i.e. that of a weakly meandering river. In this case, we derive the complete first-order expansion of the flow, that is to say we compute the first-order free surface and 
longitudinal velocity. Note that the hypothesis $R_{y}=\mathcal{O}(\varepsilon)$ is nothing but a geometric condition, satisfied as soon as the river is not strongly meandering. Then, we go back to the case $R_{y}=\mathcal{O}(1)$, but only to compute the first-order sur-elevation of the water surface.

\subsubsection{Weakly meandering case: free surface and longitudinal velocity}

Let us consider a weakly meandering river, given by $R_{y}=\mathcal{O}(\varepsilon)$. Therefore, since we still have $R_{l}=\mathcal{O}(\varepsilon)$, we get $\frac{\delta F^{2}}{J_{0}} R_{y}+R_{l}^{2}=\mathcal{O}\left(\varepsilon^{2}\right)$ and $\tilde{\varepsilon}=\mathcal{O}(\varepsilon)$. In this context, we are able to write a tractable first-order expansion of the longitudinal fluid velocity.

By rewriting the equation (3.5) and by taking into account these asymptotic expansions, we get:

$$
\frac{|\mathcal{F}|\left(v_{1}^{(0)}\left|v_{1}^{(0)}\right|-v_{1}\left|v_{1}\right|\right)}{\mathrm{C}^{2}}=\varepsilon\left(\left(v_{1}\right)_{\mathrm{t}}+v_{1}\left(v_{1}\right)_{\xi_{1}}+v_{2}\left(v_{1}\right)_{\xi_{2}}\right)+\mathcal{O}\left(\varepsilon^{2}\right)
$$

Therefore, if $v_{1}=v_{1}^{(0)}+\varepsilon v_{1}^{(1)}+\mathcal{O}\left(\varepsilon^{2}\right)$, we immediately obtain:

$$
v_{1}^{(1)}=-\frac{|\mathcal{F}|^{2} v_{1}^{(0)}}{2 \Lambda}\left(\left(v_{1}^{(0)}\right)_{t}+v_{1}^{(0)}\left(v_{1}^{(0)}\right)_{\xi_{1}}+v_{2}^{(0)}\left(v_{1}^{(0)}\right)_{\xi_{2}}\right) \text {. }
$$

Equipped with the above formula for $v_{1}^{(1)}$, we are now in a position to compute the first-order expansion of $\mathrm{Q}$, defined by $\mathrm{Q}^{(1)}=\int \Xi_{-}^{\Xi_{+}}|\mathcal{F}| \mathrm{h} \nu_{1}^{(1)} \mathrm{d} \xi_{2}$. Using the chain rule, arguing the mass conservation equation and performing straightforward computations, we get

$$
\mathrm{Q}^{(1)}=-\int_{\Xi_{-}}^{\Xi_{+}} \frac{|\mathcal{F}|^{2}}{4 \Lambda}\left(\left(|\mathcal{F}| \mathrm{h}\left(v_{1}^{(0)}\right)^{2}\right)_{t}+\left(|\mathcal{F}| \mathrm{h}\left(v_{1}^{(0)}\right)^{3}\right)_{\xi_{1}}+\left(|\mathcal{F}| \mathrm{h}\left(v_{1}^{(0)}\right)^{2} v_{2}^{(0)}\right)_{\xi_{2}}\right) \mathrm{d} \xi_{2} .
$$

Remark that $|\mathcal{F}|=1+\mathcal{O}(\varepsilon)$. Therefore, since $h\left(\Xi_{-}\right)=h\left(\Xi_{+}\right)=0$, the following expression of $\mathrm{Q}^{(1)}$ is equivalent to the above formula, up to $\mathcal{O}(\varepsilon)$ :

$$
\mathrm{Q}^{(1)}=\frac{-1}{4 \Lambda}\left[\left(\int_{\Xi_{-}}^{\Xi_{+}}|\mathcal{F}| \mathrm{h} v_{1}^{2} \mathrm{~d} \xi_{2}\right)_{\mathrm{t}}+\left(\int_{\Xi_{-}}^{\Xi_{+}}|\mathcal{F}| \mathrm{h} v_{1}^{3} \mathrm{~d} \xi_{2}\right)_{\xi_{1}}\right]+\mathcal{O}(\varepsilon) .
$$

Note that, like $\mathrm{Q}^{(0)}, \mathrm{Q}^{(1)}$ only depends on geometric quantities and on $\mathrm{H}$ (through $v_{1}^{(0)}$ ). In addition, note that the diffusive waves model $S_{t}+\left(Q^{(0)}+\varepsilon Q^{(1)}\right)_{\xi_{1}}=0$ also turns out to be nonstandard, like the kinematic waves model. Here, $Q^{(1)}$ depends on $H$, on $H_{\xi_{1}}$ through $\Lambda$, on $H_{\xi_{1} \xi_{1}}$ through $\Lambda_{\xi_{1}}$ and on $H_{\xi_{1} \xi_{1} \xi_{1}}$ through $\Lambda_{t}$, instead of only depending on $\mathrm{H}$ and $\mathrm{H}_{\xi_{1}}$ like in (3.2).

\subsubsection{Strongly meandering case: free surface sur-elevation}

In this section, we estimate the influence of the centrifugal forces on the free surface elevation. For that purpose, we consider a strongly meandering river, with $R_{y}=\mathcal{O}(1)$, and we keep $R_{l}=\mathcal{O}(\varepsilon)$. Therefore, the free surface equation (2.6) reads as follows:

$$
\frac{\delta}{J_{0}}(h+\phi)_{\xi_{2}}+R_{y} \sigma|\mathcal{F}| \varepsilon \frac{v_{1}^{2}}{R}=\mathcal{O}\left(\varepsilon F^{2}\right)
$$

Once again, we introduce the average free surface $H=H\left(\xi_{1}, t\right)$, to write:

$$
\frac{\delta}{J_{0}}(h+\phi)=\frac{\delta}{J_{0}} H-\int_{0}^{\xi_{2}} R_{y} \sigma|\mathcal{F}| \varepsilon \frac{v_{1}^{2}}{R} d \eta+\mathcal{O}\left(\varepsilon F^{2}\right) .
$$


Since $v_{1}=v_{1}^{(0)}+\mathcal{O}(\varepsilon)$, where $v_{1}^{(0)}$ is given by (3.7), we define the water surface sur-elevation $\Delta \mathrm{H}\left(\xi_{1}, \xi_{2}, \mathrm{t}\right)$ as follows:

$$
\frac{\delta}{J_{0}}(h+\phi)=\frac{\delta}{J_{0}} H+\varepsilon \Delta H+\mathcal{O}\left(\varepsilon F^{2}\right), \text { where } \Delta H=-\sigma \frac{R_{y}}{R}|\Lambda| \int_{0}^{\xi_{2}} \frac{C(H-\phi)^{2}}{|\mathcal{F}|^{2}} d \eta .
$$

We note that $\Delta \mathrm{H}$ vanishes as soon as the river is straight, i.e. when $\sigma=0$. In addition, since the only quantities whose sign is non-constant are $\xi_{2}$ and $\sigma$, we note that the sign of the free surface sur-elevation $\Delta \mathrm{H}$ given by (3.12) is the same as the sign of $-\sigma \xi_{2}$. According to figure 2, this relation means that the water surface is higher on the side of the river opposite the meander, and lower close to the meander. Indeed, it stands to reason that the centrifugal force within the meander will drive the flow towards the outer region of the meander. This behavior is consistent with the experiments and subsequent open-channel hydraulics models (see for instance [6], Page 447).

\section{Zeroth-order two-equation model for a strongly meandering river}

In this section, we assume that the river is strongly meandering, meaning that $R_{y} \leqslant \mathcal{O}(1)$, and we derive a 1D section-averaged model which is consistent up to $\mathrm{O}(\varepsilon)$ with the $2 \mathrm{D}$ shallow water system. The variables of this new model should only depend on $t$ and $\xi_{1}$, although an underlying 2D dependency will remain for known quantities (e.g. the shape of the channel or the friction distribution).

We start with the section-averaged mass and $\xi_{1}$-discharge equations $(2.9 \mathrm{a})-(2.9 \mathrm{~b})$. The mass conservation equation is already written in a $1 \mathrm{D}$ form, with the $1 \mathrm{D}$ variables $S$ and $Q$, and there is nothing further to do. To get a zeroth-order model, the discharge equation of the 1D model must be consistent with (2.9b) up to $\mathcal{O}(1)$, since $(2.9 \mathrm{~b})$ contains $\mathcal{O}\left(\frac{1}{\varepsilon}\right)$ terms. In this context, since $\Lambda$ does not depend on $\xi_{2}$, we rewrite the equation $(2.9 \mathrm{~b})$ as follows (after multiplying by $\varepsilon$ and rearranging the terms):

$$
\wedge \int_{\Xi_{-}}^{\Xi_{+}} \frac{\mathrm{h}}{|\mathcal{F}|} \mathrm{d} \xi_{2}-\int_{\Xi_{-}}^{\Xi_{+}} \frac{|\mathcal{F}|^{2} h v_{1}\left|v_{1}\right|}{\mathrm{C}^{2}} \mathrm{~d} \xi_{2}=\varepsilon\left(\mathrm{Q}_{\mathrm{t}}+\left(\int_{\Xi_{-}}^{\Xi_{+}}|\mathcal{F}| \mathrm{h} v_{1}^{2} \mathrm{~d} \xi_{2}\right)_{\xi_{1}}\right)+\mathcal{O}(\varepsilon)
$$

The higher-order terms of the above equation correspond to the geometry and friction terms, and they lie in its lefthand side. In order to write a fully 1D model, we need to replace the integrals involving the 2D variable $v_{1}$, which depends on $\xi_{2}$, by truly 1D consistent approximations. Therefore, we first derive a friction model, consistent up to $\mathcal{O}(\varepsilon)$.

We wish to approximate the left-hand side of (4.1) as follows:

$$
\wedge \int_{\Xi_{-}}^{\Xi_{+}} \frac{h}{|\mathcal{F}|} \mathrm{d} \xi_{2}-\int_{\Xi_{-}}^{\Xi_{+}} \frac{|\mathcal{F}|^{2} h v_{1}\left|v_{1}\right|}{C^{2}} \mathrm{~d} \xi_{2}=S_{m}\left(\Lambda-J_{m}\right)+\mathcal{O}(\varepsilon)
$$

where $S_{m}$ is defined by

$$
S_{\mathrm{m}}:=\int_{\Xi_{-}}^{\Xi_{+}} \frac{\mathrm{h}}{|\mathcal{F}|} \mathrm{d} \xi_{2}
$$

and where $J_{m}$ is a $1 \mathrm{D}$ friction model, which we take according to the usual hydraulic engineering expressions:

$$
\mathcal{J}_{\mathrm{m}}:=\frac{\mathrm{Q}|\mathrm{Q}|}{\left(\mathrm{C}_{\mathrm{h}}\right)_{\mathrm{m}}^{2} \mathrm{R}_{\mathrm{h}} \mathrm{S}^{2}},
$$

where $\left(C_{h}\right)_{m}$ is a 1D friction coefficient whose expression will be computed to ensure consistency.

By combining (4.1) and (4.2) with the expression of $\mathcal{J}_{m}$, we obtain that the friction coefficient $\left(C_{h}\right)_{m}$ must satisfy the constraint

$$
\Lambda=\frac{\mathrm{Q}|\mathrm{Q}|}{\left(\mathrm{C}_{\mathrm{h}}\right)_{\mathrm{m}}^{2} \mathrm{R}_{\mathrm{h}} \mathrm{S}^{2}}+\mathcal{O}(\varepsilon)
$$


Since we also want to ensure $\mathrm{Q}=\mathrm{Q}^{(0)}+\mathrm{O}(\varepsilon)$ for our model to be consistent up to $\mathcal{O}(\varepsilon)$, where $\mathrm{Q}^{(0)}$ is given by (3.9), we can replace $Q$ with $Q^{(0)}$ in the above equality, to choose the following parameterization of the friction coefficient:

$$
\left(C_{h}\right)_{m}^{2}=\frac{Q^{(0)}\left|Q^{(0)}\right|}{R_{h} S^{2} \Lambda}=\frac{1}{R_{h} S^{2}}\left(\int_{\Xi_{-}}^{\Xi_{+}} \frac{h C}{\sqrt{|\mathcal{F}|}} d \xi_{2}\right)^{2} .
$$

Note that, despite the expression of $\mathrm{Q}^{(0)}$ containing the differential term $\mathrm{H}_{\xi_{1}}$, present in $\Lambda$, the resulting friction coefficient $\left(C_{h}\right)_{m}$ does not contain any differential terms. It is therefore a suitable component of the friction source term. We also note that the 1D friction model reads as follows:

$$
\partial_{\mathrm{m}}=\Lambda \frac{\mathrm{Q}|\mathrm{Q}|}{\mathrm{Q}^{(0)}\left|\mathrm{Q}^{(0)}\right|}=\mathrm{Q}|\mathrm{Q}|\left(\int_{\Xi_{-}}^{\Xi_{+}} \frac{\mathrm{hC}}{\sqrt{|\mathcal{F}|}} \mathrm{d} \xi_{2}\right)^{-2} .
$$

The discharge equation (4.1) then rewrites:

$$
\mathrm{Q}_{\mathrm{t}}+\left(\int_{\Xi_{-}}^{\Xi_{+}}|\mathcal{F}| \mathrm{h} v_{1}^{2} \mathrm{~d} \xi_{2}\right)_{\xi_{1}}=\frac{1}{\varepsilon} \mathrm{S}\left(\Lambda-\mathcal{J}_{\mathrm{m}}\right)+\mathcal{O}(1) .
$$

The $\Lambda$ in the source term contains the differential term $\mathrm{H}_{\xi_{1}}$, which should be contained in the flux. Introducing the longitudinal slope $\mathcal{J}$ as

$$
\mathcal{J}=-\frac{I_{0}}{J_{0}} b_{0}^{\prime}
$$

we note that $\Lambda=\mathcal{J}-\frac{\delta}{J_{0}} H_{\xi_{1}}$, and we are thus able to group the differential terms in the left-hand side of (4.5). Finally, by analogy with the classical shallow water system in $(S, Q)$ variables, let us approximate $\int \Xi_{-}^{\Xi_{+}}|\mathcal{F}| h v_{1}^{2} \mathrm{~d} \xi_{2}$ with $\frac{\mathrm{Q}^{2}}{\mathrm{~S}}$, which is valid up to $\mathcal{O}(1)$. The complete zeroth-order $1 \mathrm{D}$ model for a meandering river thus reads:

$$
\left\{\begin{array}{l}
S_{t}+Q_{x}=0 \\
Q_{t}+\left(\frac{Q^{2}}{S}\right)_{\xi_{1}}+\frac{S H_{\xi_{1}}}{F^{2}}=\frac{1}{\varepsilon} S\left(J-J_{m}\right) .
\end{array}\right.
$$

This is the canonical form of section-averaged shallow water equations found in the literature: see for instance $[6,36]$ and references therein. We removed $\mathcal{O}(1)$ terms accounting for the influence of the meandering in order to get a simple formulation of the shallow water model. However, the form of the source term ensures the consistency of this shallow water model up to order $\mathcal{O}(\varepsilon)$ with the $2 \mathrm{D}$ shallow water equations, which is far from being obvious for other similar models. By construction, our model conserves the fluid mass and is consistent up to $\mathcal{O}(\varepsilon)$ with the $2 \mathrm{D}$ model in the asymptotic regime under consideration. In addition, its homogeneous form is hyperbolic, by analogy with the classical shallow water model in $(S, Q)$ variables.

Thus, the complexity of the model does not lie in the form of the equations (4.6), which are nothing but the classical mass and discharge equations of the usual section-averaged shallow water equations. Rather, the complexity is in the expression of the friction model $\mathcal{J}_{m}$, and more specifically in the friction coefficient $\left(C_{h}\right)_{m}$, given by (4.3). For U-shaped channels with uniform friction, straightforward computations show that this friction coefficient is equivalent to the usual hydraulic engineering ones. For a non-uniform geometry or friction, this zeroth-order coefficient gives a new formula to derive a 1D friction model. In any case, this friction coefficient ensures that the uniform and stationary flows are correctly captured by the model (4.6).

Even though this model does not capture the first-order variations of the section-averaged discharge, it is able to take into account the water surface sur-elevation. Indeed, considering the definition (2.10) of the wetted 
section $S=\int \Xi_{-}^{\Xi_{+}}|\mathcal{F}| \mathrm{h} \mathrm{d} \xi_{2}$, we get:

$$
S=H \int_{\Xi_{-}}^{\Xi_{+}}|\mathcal{F}| \mathrm{d} \xi_{2}-\int_{\Xi_{-}}^{\Xi_{+}}|\mathcal{F}| \phi d \xi_{2}+\mathrm{F}^{2} \int_{\Xi_{-}}^{\Xi_{+}}|\mathcal{F}| \Delta \mathrm{Hd} \xi_{2}+\mathcal{O}\left(\varepsilon \mathrm{F}^{2}\right),
$$

where the sur-elevation $\Delta \mathrm{H}$ is given by (3.12). All the terms in the above equality are geometrical, so it is possible to compute $\mathrm{S}$ from $\mathrm{H}$ and $\mathrm{H}$ from $\mathrm{S}$ up to $\mathcal{O}\left(\varepsilon \mathrm{F}^{2}\right)$. However, in general, the computations require a nonlinear solver since $\Delta \mathrm{H}$ depends non-linearly on $\mathrm{H}$. Let us note that, for a $\mathrm{U}$-shaped channel, the following equality holds:

$$
|\mathcal{F}| \Delta H=-\sigma \frac{R_{y}}{R}|\Lambda| C(H) \xi_{2} .
$$

Thus, we immediately get, for a U-shaped channel where $\Xi_{-}=-\Xi_{+}$,

$$
\int_{\Xi_{-}}^{\Xi_{+}}|\mathcal{F}| \Delta \mathrm{Hd} \xi_{2}=0
$$

The wetted section given by (4.7) is then very easy to compute for a U-shaped channel. Furthermore, tests made on several channel shapes (circular, trapezoidal, ...) have all shown that the $\int_{\Xi_{-}}^{\Xi_{+}}|\mathcal{F}| \Delta \mathrm{H} d \xi_{2}$ is always very small. It should therefore be safe to neglect this term even for non-U-shaped channels. As a consequence, the 1D model (4.6), despite being only zeroth-order accurate on the averaged discharge, is able to compute the water surface sur-elevation up to first-order accuracy with an a posteriori treatment of the zeroth-order free surface.

\section{First-order four-equation model for a weakly meandering river}

We now assume that the river is weakly meandering, meaning that $R_{y}=\mathcal{O}(\varepsilon)$. The goal of this section is to derive a suitable 1D model, consistent up to first-order (i.e. up to $\mathcal{O}\left(\varepsilon^{2}\right)$ ) with the 2D system $(2.4)-(2.7)$. Similarly to the strongly meandering case, the variables of this new model should only depend on $t$ and $\xi_{1}$.

To that end, we start with the section-averaged system (2.9), and we introduce a relevant friction model as well as new 1D variables. Then, we propose an Euler-like four-equation model to satisfy the asymptotic regime under consideration. Finally, we exhibit some mathematical properties satisfied by this new system.

\subsection{Consistent section-averaged system}

To consider a first-order model, we set the forthcoming developments in the context of section 3.2, i.e. we assume that $R_{l}=\mathcal{O}(\varepsilon)$ and $R_{y}=\mathcal{O}(\varepsilon)$. In this case, we have $|\mathcal{F}|=1+\mathcal{O}\left(R_{y}\right)=1+\mathcal{O}(\varepsilon)$, and thus $|\mathcal{F}| \mathrm{h}=h+\mathcal{O}(\varepsilon)$. These remarks allow us to write the following asymptotic expansion of the integrated system (2.9):

$$
\left\{\begin{array}{l}
\mathrm{S}_{\mathrm{t}}+\mathrm{Q}_{\xi_{1}}=0, \\
\mathrm{Q}_{\mathrm{t}}+\left(\int_{\Xi_{-}}^{\Xi_{+}}|\mathcal{F}| \mathrm{h} v_{1}^{2} \mathrm{~d} \xi_{2}\right)_{\xi_{1}}=\frac{1}{\varepsilon}\left(\Lambda \int_{\Xi_{-}}^{\Xi_{+}} \frac{\mathrm{h}}{|\mathfrak{F}|} \mathrm{d} \xi_{2}-\int_{\Xi_{-}}^{\Xi_{+}} \frac{|\mathcal{F}|^{2} h v_{1}\left|v_{1}\right|}{\mathrm{C}^{2}} \mathrm{~d} \xi_{2}\right)+\mathcal{O}(\varepsilon), \\
\left(\int_{\Xi_{-}}^{\Xi_{+}} \frac{1}{2}|\mathcal{F}| \mathrm{h} v_{1}^{2} \mathrm{~d} \xi_{2}\right)_{\mathrm{t}}+\left(\int_{\Xi_{-}}^{\Xi_{+}} \frac{1}{2}|\mathcal{F}| \mathrm{h} v_{1}^{3} \mathrm{~d} \xi_{2}\right)_{\xi_{1}}=\frac{1}{\varepsilon}\left(\Lambda \mathrm{Q}-\int_{\Xi_{-}}^{\Xi_{+}} \frac{\mathrm{h} v_{1}|\mathcal{F}|^{4} v_{1}\left|v_{1}\right|}{\mathrm{C}^{2}} \mathrm{~d} \xi_{2}\right)+\mathcal{O}(\varepsilon) .
\end{array}\right.
$$

These equations can be rewritten to introduce the so-called velocity-distribution terms $\alpha$ and $\beta$, see for instance [6], Page 27. The reader is also referred, for instance, to $[40,13,16]$ for a discussion on the asymmetry 
of the velocity flow in straight and curved channels. The Boussinesq coefficient $\beta$ is a correction term in the discharge flux, and the Coriolis coefficient $\alpha$ is a correction of the energy flux. These dimensionless coefficients are defined as the following ratios:

$$
\beta=\frac{\int_{\Xi_{-}}^{\Xi_{+}}|\mathcal{F}| \mathrm{h} v_{1}^{2} \mathrm{~d} \xi_{2}}{\frac{1}{\mathrm{~S}}\left(\int_{\Xi_{-}}^{\Xi_{+}}|\mathcal{F}| \mathrm{h} \nu_{1} \mathrm{~d} \xi_{2}\right)^{2}}=\frac{\int_{\Xi_{-}}^{\Xi_{+}}|\mathcal{F}| \mathrm{h} v_{1}^{2} \mathrm{~d} \xi_{2}}{\frac{\mathrm{Q}^{2}}{\mathrm{~S}}} \text { and } \alpha=\frac{\int_{\Xi_{-}}^{\Xi_{+}}|\mathcal{F}| \mathrm{h} v_{1}^{3} \mathrm{~d} \xi_{2}}{\frac{1}{\mathrm{~S}^{2}}\left(\int_{\Xi_{-}}^{\Xi_{+}}|\mathcal{F}| \mathrm{h} \nu_{1} \mathrm{~d} \xi_{2}\right)^{3}}=\frac{\int_{\Xi_{-}}^{\Xi_{+}}|\mathcal{F}| \mathrm{h} v_{1}^{3} \mathrm{~d} \xi_{2}}{\frac{\mathrm{Q}^{3}}{\mathrm{~S}^{2}}}
$$

Introducing the averaged velocity $\mathrm{U}$ defined by $\mathrm{Q}=\mathrm{SU}$, the system (5.1) can thus be rewritten with the velocitydistribution coefficients, as follows:

$$
\left\{\begin{array}{l}
\mathrm{S}_{\mathrm{t}}+\mathrm{Q}_{\xi_{1}}=0, \\
\mathrm{Q}_{\mathrm{t}}+\left(\beta \mathrm{SU} \mathrm{U}^{2}\right)_{\xi_{1}}=\frac{1}{\varepsilon}\left(\Lambda \int_{\Xi_{-}}^{\Xi_{+}} \frac{\mathrm{h}}{|\mathfrak{F}|} \mathrm{d} \xi_{2}-\int_{\Xi_{-}}^{\Xi_{+}} \frac{|\mathcal{F}|^{2} h v_{1}\left|v_{1}\right|}{\mathrm{C}^{2}} \mathrm{~d} \xi_{2}\right)+\mathcal{O}(\varepsilon), \\
\left(\frac{1}{2} \beta S \mathrm{SU}^{2}\right)_{\mathrm{t}}+\left(\frac{1}{2} \alpha \mathrm{SU}^{3}\right)_{\xi_{1}}=\frac{1}{\varepsilon}\left(\Lambda \mathrm{Q}-\int_{\Xi_{-}}^{\Xi_{+}} \frac{\mathrm{h} v_{1}|\mathcal{F}|^{4} v_{1}\left|v_{1}\right|}{\mathrm{C}^{2}} \mathrm{~d} \xi_{2}\right)+\mathcal{O}(\varepsilon) .
\end{array}\right.
$$

Usually, the terms $\alpha$ and $\beta$ are either given an empiric constant value, which depends on the shape of the channel (see [6], Page 28), or are approximated using the velocity distribution in the flow (see again [6], Page 29). Instead, we consider these terms as unknowns in the forthcoming developments, and we provide analytic expressions for their asymptotic expansions.

The goal of the remainder of this section is to propose a 1D model that is first-order accurate with respect to the small parameter $\varepsilon$. That is to say, contrary to section 4 where we had the zeroth-order expansion $\mathrm{Q}=\mathrm{Q}^{(0)}+\mathcal{O}(\varepsilon)$, we now wish to recover a higher accuracy: $Q=Q^{(0)}+\varepsilon Q^{(1)}+\mathcal{O}\left(\varepsilon^{2}\right)$.

In order to address this issue, we first consider a zeroth-order approximation of the discharge equation, like in section 4 . This approximation is obtained by defining a relevant friction model. Equipped with this friction model, we turn to providing a consistent approximation of the energy equation up to first-order in $\varepsilon$. Finally, we propose a conservative form of the proposed first-order system.

\subsubsection{The friction model}

In order to get a 1D system from the integrated equations (5.1), we introduce a model for the friction term, consistent with the standard engineering ones. Starting with the discharge equation (5.1b), we seek a 1D friction model that is consistent with the $2 \mathrm{D}$ friction term up to $\mathcal{O}(\varepsilon)$. We now proceed in a similar way as in section 4 . Noting that a zeroth-order expansion of the integrated discharge equation (5.1b) reads as follows:

$$
\mathrm{Q}_{\mathrm{t}}+\left(\int_{\Xi_{-}}^{\Xi_{+}}|\mathcal{F}| \mathrm{h} v_{1}^{2} \mathrm{~d} \xi_{2}\right)_{\xi_{1}}=\frac{1}{\varepsilon}\left(\Lambda \mathrm{S}-\int_{\Xi_{-}}^{\Xi_{+}} \frac{|\mathcal{F}|^{2} h v_{1}\left|v_{1}\right|}{\mathrm{C}^{2}} \mathrm{~d} \xi_{2}\right)+\mathcal{O}(1),
$$

we seek $C_{h}$ such that

$$
\int_{\Xi_{-}}^{\Xi_{+}} \frac{|\mathcal{F}|^{2} h v_{1}\left|v_{1}\right|}{C^{2}} d \xi_{2}=S \frac{Q|Q|}{C_{h}^{2} R_{h} S^{2}}+\mathcal{O}(\varepsilon)=: S \mathcal{J}+\mathcal{O}(\varepsilon)
$$

Like in section 4 , it is sufficient to choose $C_{h}$ such that $\Lambda=\frac{Q^{(0)}\left|Q^{(0)}\right|}{C_{h}^{2} R_{h} S^{2}}$, and we get the same friction coefficient:

$$
C_{h}^{2}=\frac{Q^{(0)}\left|Q^{(0)}\right|}{R_{h} S^{2} \Lambda}=\frac{1}{R_{h} S^{2}}\left(\int_{\Xi_{-}}^{\Xi_{+}} \frac{h C}{\sqrt{|\mathcal{F}|}} d \xi_{2}\right)^{2} \text {. }
$$


We then rewrite the discharge equation $(2.9 \mathrm{~b})$ as follows:

$$
\mathrm{Q}_{\mathrm{t}}+\left(\int_{\Xi_{-}}^{\Xi_{+}}|\mathcal{F}| \mathrm{h} v_{1}^{2} \mathrm{~d} \xi_{2}\right)_{\xi_{1}}=\frac{1}{\varepsilon} \mathrm{S}(\Lambda-\mathcal{J})+\mathcal{O}(1)
$$

The term $\Lambda$ of this equation contains the differential term $\mathrm{H}_{\xi_{1}}$, which should be included in the flux rather than in the source term. This discrepancy will be dealt with in a forthcoming section.

Let us note that $\mathcal{J}$ does not contain any differential term coming from $\Lambda$, even though $\Lambda$ contains $\mathrm{H}_{\xi_{1}}$. Indeed, since $C_{h}$ is given by (5.3) and $Q^{(0)}$ is given by (3.9), we obtain the same friction term (4.4) as in the strongly meandering case:

$$
\mathcal{J}=\Lambda \frac{\mathrm{Q}|\mathrm{Q}|}{\mathrm{Q}^{(0)} \mid \mathrm{Q}^{(0) \mid}}=\mathrm{Q}|\mathrm{Q}|\left(\int_{\Xi_{-}}^{\Xi_{+}} \frac{h \mathrm{C}}{\sqrt{|\mathcal{F}|}} \mathrm{d} \xi_{2}\right)^{-2}
$$

\subsubsection{Consistent energy equation}

The discharge equation (5.4), thanks to the friction model (5.3), correctly recovers the zeroth-order asymptotic expansion $\mathrm{Q}=\mathrm{Q}^{(\mathcal{O})}+\mathcal{O}(\varepsilon)$ when $\varepsilon \rightarrow O$. As a consequence, plugging this friction model into the classical shallow water system would be sufficient to ensure the accuracy of this asymptotic expansion. However, we wish to go one step further and actually get accuracy up to the first-order asymptotic expansion

$$
\mathrm{Q}=\mathrm{Q}^{(0)}+\varepsilon \mathrm{Q}^{(1)}+\mathcal{O}\left(\varepsilon^{2}\right)
$$

where $\mathrm{Q}^{(1)}$ is given by (3.11). The mathematical structure of the discharge equation is well-understood and ensures essential properties, such as hyperbolicity. Therefore, we do not wish to modify this equation. To address this issue, we elect to add a new equation to the model. Indeed, we choose to deriving a suitable approximation of the integrated energy equation (2.9c) in order to achieve the more accurate first-order approximation of the averaged discharge. Such an approach was already suggested to derive consistent shallow water models for thin film flows down an incline [21].

In order to mimic the structure of Euler equations for numerical simulation purpose and ensure a good mathematical structure, we search for an averaged energy equation that could be derived from the momentum equation. Thus, we search for a source term in the energy equation that is the product of the source term in the momentum equation with the discharge rate. The source term of this new energy balance law, plugging the asymptotic expansion (3.9) - (3.11) into the expression (5.5) of the friction model $\mathcal{J}$, satisfies:

$$
\frac{1}{\varepsilon} \mathrm{Q}(\Lambda-\mathcal{J})=-2 \wedge \mathrm{Q}^{(1)}+\mathcal{O}(\varepsilon) .
$$

Since $\mathrm{Q}^{(1)}$ is given by (3.11), the new energy equation reads

$$
\left(\frac{1}{2} \int_{\Xi_{-}}^{\Xi_{+}}|\mathcal{F}| \mathrm{h} v_{1}^{2} \mathrm{~d} \xi_{2}\right)_{\mathrm{t}}+\left(\frac{1}{2} \int_{\Xi_{-}}^{\Xi_{+}}|\mathcal{F}| \mathrm{h} v_{1}^{3} \mathrm{~d} \xi_{2}\right)_{\xi_{1}}=\frac{1}{\varepsilon} \mathrm{Q}(\Lambda-\mathcal{J})+\mathcal{O}(\varepsilon) .
$$

This energy equation is consistent with the averaged energy equation (5.1c) up to $\mathcal{O}(\varepsilon)$. 


\subsubsection{Final conservative form of the integrated $2 D$ system}

Thanks to the friction model (5.3) and therefore the equations (5.4) and (5.7), the system (5.1) rewrites:

$$
\left\{\begin{array}{l}
\mathrm{S}_{\mathrm{t}}+\mathrm{Q}_{\xi_{1}}=0, \\
\mathrm{Q}_{\mathrm{t}}+\left(\int_{\Xi_{-}}^{\Xi_{+}}|\mathcal{F}| \mathrm{h} v_{1}^{2} \mathrm{~d} \xi_{2}\right)_{\xi_{1}}=\frac{1}{\varepsilon} \mathrm{S}(\Lambda-\mathcal{J})+\mathcal{O}(1) \\
\left(\frac{1}{2} \int_{\Xi_{-}}^{\Xi_{+}}|\mathcal{F}| \mathrm{h} v_{1}^{2} \mathrm{~d} \xi_{2}\right)_{\mathrm{t}}+\left(\frac{1}{2} \int_{\Xi_{-}}^{\Xi_{+}}|\mathcal{F}| \mathrm{h} \nu_{1}^{3} \mathrm{~d} \xi_{2}\right)_{\xi_{1}}=\frac{1}{\varepsilon} \mathrm{Q}(\Lambda-\mathcal{J})+\mathcal{O}(\varepsilon) .
\end{array}\right.
$$

Compared to the integrated 2D equations (5.1), the momentum equation has a $\mathcal{O}(1)$ deviation and the energy equation has a $\mathcal{O}(\varepsilon)$ deviation. Nevertheless, these deviations are enough to ensure that the asymptotic expansion of $\mathrm{Q}$ is satisfied up to $\mathcal{O}\left(\varepsilon^{2}\right)$ : we get $\mathrm{Q}=\mathrm{Q}^{(0)}+\varepsilon \mathrm{Q}^{(1)}+\mathcal{O}\left(\varepsilon^{2}\right)$ when $\varepsilon \rightarrow 0$.

Note that $\Lambda$ contains the space derivative $\mathrm{H}_{\xi_{1}}$. No differential term should remain in the source; rather, they should be regrouped in the flux to get a good mathematical structure. Since $\Lambda$ is given by (3.8), the system (5.8) is equivalent to

$$
\left\{\begin{array}{l}
\mathrm{S}_{\mathrm{t}}+\mathrm{Q}_{\xi_{1}}=0, \\
\mathrm{Q}_{\mathrm{t}}+\left(\int_{\Xi_{-}}^{\Xi_{+}}|\mathcal{F}| \mathrm{h} v_{1}^{2} \mathrm{~d} \xi_{2}\right)_{\xi_{1}}+\frac{\mathrm{SH}_{\xi_{1}}}{\mathrm{~F}^{2}}=\frac{1}{\mathcal{\varepsilon}} \mathrm{S}(\mathcal{J}-\mathcal{J})+\mathcal{O}(1), \\
\left(\frac{1}{2} \int_{\Xi_{-}}^{\Xi_{+}}|\mathcal{F}| \mathrm{h} v_{1}^{2} \mathrm{~d} \xi_{2}\right)_{\mathrm{t}}+\left(\frac{1}{2} \int_{\Xi_{-}}^{\Xi_{+}}|\mathcal{F}| \mathrm{h} v_{1}^{3} \mathrm{~d} \xi_{2}\right)_{\xi_{1}}+\frac{\mathrm{QH} \xi_{1}}{\mathrm{~F}^{2}}=\frac{1}{\varepsilon} \mathrm{Q}(\mathcal{J}-\mathcal{J})+\mathcal{O}(\varepsilon) .
\end{array}\right.
$$

The above system can be recast under a conservative form. Let us define the width function $L\left(\xi_{1}, z, t\right)$ such that $S\left(\xi_{1}, t\right)=\int_{0}^{H\left(\xi_{1}, t\right)} L\left(\xi_{1}, z, t\right) d z$, see figure 1. First, note the following chain of equalities:

$$
\frac{\mathrm{SH}_{\xi_{1}}}{\mathrm{~F}^{2}}=\frac{\mathrm{H}_{\xi_{1}}}{\mathrm{~F}^{2}} \int_{0}^{\mathrm{H}} \mathrm{Ld} z=\left(\frac{1}{\mathrm{~F}^{2}} \int_{0}^{\mathrm{H}}(\mathrm{H}-z) \mathrm{Ld} z\right)_{\xi_{1}}-\frac{1}{\mathrm{~F}^{2}} \int_{0}^{\mathrm{H}}(\mathrm{H}-z) \mathrm{L}_{\xi_{1}} \mathrm{~d} z=: \mathrm{P}_{\xi_{1}}^{\text {hy }}-\mathrm{P}^{\text {lat }},
$$

where $\mathrm{P}^{\text {hy }}$ is the hydrostatic pressure and $\mathrm{P}^{\text {lat }}$ is the lateral pressure, see [38]. In addition, remark that

$$
\frac{\mathrm{QH}_{\xi_{1}}}{\mathrm{~F}^{2}}=\frac{\mathrm{HS} \mathrm{t}_{\mathrm{t}}+(\mathrm{HQ}) \xi_{1}}{\mathrm{~F}^{2}}=\left(\frac{1}{\mathrm{~F}^{2}} \int_{0}^{\mathrm{H}} z \mathrm{Ld} z\right)_{\mathrm{t}}+\left(\frac{\mathrm{U}}{\mathrm{F}^{2}}\left(\int_{0}^{\mathrm{H}} \mathrm{HLd} \mathrm{d}\right)\right)_{\xi_{1}}=: \mathcal{E}_{\mathrm{t}}+\left(\mathrm{U}\left(\mathcal{E}+\mathrm{P}^{\mathrm{hy}}\right)\right)_{\xi_{1}},
$$

where $\mathcal{E}$ is the potential gravity energy, and where the averaged flow velocity $\mathrm{U}$ is defined by $\mathrm{U}=\mathrm{Q} / \mathrm{S}$. Therefore, the system (5.9) reads:

$$
\left\{\begin{array}{l}
\mathrm{S}_{\mathrm{t}}+\mathrm{Q}_{\xi_{1}}=0, \\
\mathrm{Q}_{\mathrm{t}}+\left(\mathrm{P}^{\mathrm{hy}}+\int_{\Xi_{-}}^{\Xi_{+}}|\mathcal{F}| \mathrm{h} v_{1}^{2} \mathrm{~d} \xi_{2}\right)_{\xi_{1}}=\mathrm{P}^{\text {lat }}+\frac{1}{\varepsilon} \mathrm{S}(\mathcal{J}-\mathcal{J})+\mathcal{O}(1), \\
\left(\mathcal{E}+\frac{1}{2} \int_{\Xi_{-}}^{\Xi_{+}}|\mathcal{F}| \mathrm{h} v_{1}^{2} \mathrm{~d} \xi_{2}\right)_{\mathrm{t}}+\left(\mathcal{E}+\mathrm{P}^{\mathrm{hy}}+\frac{1}{2} \int_{\Xi_{-}}^{\Xi_{+}}|\mathcal{F}| \mathrm{h} v_{1}^{3} \mathrm{~d} \xi_{2}\right)_{\xi_{1}}=\frac{1}{\varepsilon} \mathrm{Q}(\mathcal{J}-\mathcal{J})+\mathcal{O}(\varepsilon) .
\end{array}\right.
$$

The above equations are written under the form of a system of balance laws, where no differential terms subsist in the source term. They are consistent with the width-integrated equations (5.1) and they ensure the first-order asymptotic expansion of the discharge $\mathrm{Q}=\mathrm{Q}^{(0)}+\varepsilon \mathrm{Q}^{(1)}+\mathcal{O}\left(\varepsilon^{2}\right)$ when $\varepsilon \rightarrow 0$. 


\subsection{An Euler-like model}

We now wish, in the spirit of [37], to recast the above 1D system under an Euler-like formulation to eliminate the integral terms and to ensure relevant mathematical properties, such as hyperbolicity. We first introduce a relevant energy and pressure. Then, we add a fourth equation to the model to account for the temporal variations of the pressure.

We briefly recall the classical form of the 1D homogeneous Euler system, describing fluid dynamics. In this analogy, the section $S$ stands for the density (usually denoted by $\rho$ ) in the Euler equations, and we get:

$$
\left\{\begin{array}{l}
S_{t}+(S U)_{\xi_{1}}=0, \\
(S U)_{t}+\left(S U^{2}+P\right)_{\xi_{1}}=0 \\
E_{t}+(U(E+P))_{\xi_{1}}=0
\end{array}\right.
$$

where $\mathrm{U}=\mathrm{Q} / \mathrm{S}$ is the velocity, $\mathrm{E}$ is the energy and $\mathrm{P}$ is the pressure, usually given by a pressure law $\mathrm{P}(\mathrm{S}, \mathrm{U}, \mathrm{E})$. A well-known example of a pressure law is the ideal gas law, defined by

$$
\mathrm{P}=(\gamma-1)\left(\mathrm{E}-\frac{\mathrm{SU}^{2}}{2}\right), \quad \text { with } \gamma \geqslant 1 .
$$

\subsubsection{Introduction of an energy and a pressure}

The mass conservation equation (5.10a) is exact and similar to that of the Euler system, so no work needs to be done on this equation. Next, consider the discharge balance equation (5.10b). To recover an Euler-like formulation, we introduce a pressure $P$ such that

$$
\mathrm{SU}^{2}+\mathrm{P}=\int_{\Xi_{-}}^{\Xi_{+}}|\mathcal{F}| \mathrm{h} v_{1}^{2} \mathrm{~d} \xi_{2}+\mathrm{P}^{\mathrm{hy}}
$$

As a consequence, it is natural to introduce a new variable $\Psi$, the so-called enstrophy (see for instance $[29,32,30]$ ), which corresponds to the variance of the velocity with respect to the mean flow. The enstrophy $\Psi$ is therefore defined as follows:

$$
S \Psi=\int_{\Xi_{-}}^{\Xi_{+}}|\mathcal{F}| \mathrm{h} v_{1}^{2} \mathrm{~d} \xi_{2}-S \mathrm{U}^{2}
$$

The pressure P defined by (5.12) becomes

$$
\mathrm{P}=\mathrm{P}^{\text {hy }}+\mathrm{S} \Psi .
$$

By inspection of the time derivative within the energy balance equation (5.10c), we propose the following natural definition of the energy:

$$
E=\mathcal{E}+\frac{1}{2} S U^{2}+\frac{1}{2} S \Psi
$$

such that the time derivative is applied to $E$. To take care of the spatial derivative in this equation, we introduce the variable $\Pi$, taking inspiration from the definition (5.13) of $\Psi$ :

$$
\mathrm{S} \Pi=\frac{1}{\mathrm{U}} \int_{\Xi_{-}}^{\Xi_{+}}|\mathcal{F}| \mathrm{h} v_{1}^{3} \mathrm{~d} \xi_{2}-\mathrm{SU}^{2} .
$$

In order to better understand the roles played by the new variables $\Psi$ and $\Pi$, let us rewrite each of them as a single integral. Straightforward computations yield the following expressions:

$$
S \Psi=\int_{\Xi_{-}}^{\Xi_{+}}|\mathcal{F}| \mathrm{h}\left(v_{1}-\mathrm{U}\right)^{2} \mathrm{~d} \xi_{2} \quad \text { and } \quad S \Pi=\int_{\Xi_{-}}^{\Xi_{+}}|\mathcal{F}| \mathrm{h}\left(v_{1}-\mathrm{U}\right)^{2}\left(2+\frac{v_{1}}{\mathrm{U}}\right) \mathrm{d} \xi_{2} .
$$


The enstrophy $\Psi$ accounts for the vorticity of the flow in the cross-stream direction: it is the variance of the fluid velocity $u$ in the cross-stream direction. The variable $\Pi$ plays the role of and will be referred to as a potential. Additionally, recall the expressions of the Coriolis coefficient $\alpha$ and the Boussinesq coefficient $\beta$, defined by (5.2). Straightforward computations show that we can rewrite the enstrophy and potential as follows:

$$
\frac{\mathrm{S} \Psi}{\mathrm{SU}^{2}}=\beta-1 \text { and } \quad \frac{\mathrm{S} \Pi}{\mathrm{SU}^{2}}=\alpha-1 .
$$

Recall that $\alpha \geqslant 1$ and $\beta \geqslant 1$ in the literature (see for instance [6], Page 29), where the equality case corresponds to a uniform cross-section. We thus get that $\Psi \geqslant 0$ and $\Pi \geqslant 0$, and that these quantities vanish in the case of a uniform cross-section.

According to these definitions, we suggest the following fully $1 \mathrm{D}$ model to replace the averaged 2D system (5.10):

$$
\left\{\begin{array}{l}
S_{\mathrm{t}}+\mathrm{Q}_{\xi_{1}}=0, \\
\mathrm{Q}_{\mathrm{t}}+\left(\frac{\mathrm{Q}^{2}}{\mathrm{~S}}+\mathrm{P}\right)_{\xi_{1}}=\mathrm{P}^{\text {lat }}+\frac{1}{\varepsilon} \mathrm{S}(\mathcal{J}-\mathcal{J})+\mathcal{O}(1), \\
\mathrm{E}_{\mathrm{t}}+\left(\frac{\mathrm{Q}}{\mathrm{S}}(\mathrm{E}+\mathrm{P})\right)_{\xi_{1}}+\left(\frac{1}{2} \frac{\mathrm{Q}}{\mathrm{S}} \mathrm{S}(\Pi-3 \Psi)\right)_{\xi_{1}}=\frac{1}{\varepsilon} \mathrm{Q}(\mathcal{J}-\mathcal{J})+\mathcal{O}(\varepsilon) .
\end{array}\right.
$$

An interesting remark we make here concerns the relationship between $\alpha$ and $\beta$. Indeed, one can show that, under some assumptions (see [6], Page 29) in the velocity distribution, we get $\alpha-1 \simeq 3 \mathscr{V}^{2}-2 \mathscr{V}^{3}$ and $\beta-1 \simeq \mathscr{V}^{2}$, where $\mathscr{V}=\frac{1}{\mathrm{u}} \max _{\xi_{2}} v_{1}$ is the ratio between the maximum velocity and its average. This parameter $\mathscr{V}$ vanishes in the case of a straight $U$-shaped channel, and is positive otherwise. Then, we note that the term $S(\Pi-3 \Psi)$ in $(5.14)$, or equivalently $(\alpha-1)-3(\beta-1) \simeq-2 \mathscr{V}^{3}$, represents the fact that we consider the third-order moment of the velocity to be nonzero.

At this level, we focus on the homogeneous part of the model (5.14), in order to obtain a hyperbolic system. Recall that both E and P contain the enstrophy $\Psi$. Therefore, the enstrophy is a natural variable of the model (5.14). However, a rule to compute the potential $\Pi$ is still missing to close (5.14). The goal of section 5.2.2 is to propose such a rule while ensuring that the system becomes hyperbolic. Afterwards, in section 5.2.3, we will further modify the system to ensure the required asymptotic expansion (5.6).

\subsubsection{A four-equation Euler-like model}

The time evolution of the potential $\Pi$ is not prescribed by the three-equation system (5.14). A first idea could be to impose $\Pi=\Pi^{(0)}$. However, this would lead to a model that is not hyperbolic. Therefore, we need to add an equation to this system, whose time derivative contains $\Pi$. In addition, compared to a classical Euler system, the energy equation of (5.14) has an extra differential term. To treat this term, let us denote by e the following quantity:

$$
e=\frac{1}{2} S(\Pi-3 \Psi)
$$

It becomes natural to add to the system an equation prescribing the time evolution of $e$. For the sake of simplicity, we choose to add the equation $e_{t}=\mathcal{O}(1)$. Adding this equation to the system (5.14) yields the following four- 
equation model:

$$
\left\{\begin{array}{l}
S_{\mathrm{t}}+\mathrm{Q}_{\xi_{1}}=0 \\
\mathrm{Q}_{\mathrm{t}}+\left(\frac{\mathrm{Q}^{2}}{\mathrm{~S}}+\mathrm{P}\right)_{\xi_{1}}=\mathrm{P}^{\mathrm{lat}}+\frac{1}{\varepsilon} \mathrm{S}(\mathcal{J}-\mathcal{J})+\mathcal{O}(1) \\
(\mathrm{E}+e)_{\mathrm{t}}+\left(\frac{\mathrm{Q}}{\mathrm{S}}(\mathrm{E}+e+\mathrm{P})\right)_{\xi_{1}}=\frac{1}{\varepsilon} \mathrm{Q}(\mathcal{J}-\mathcal{J})+\mathcal{O}(\varepsilon) \\
e_{\mathrm{t}}=\mathcal{O}(1)
\end{array}\right.
$$

The homogeneous part of the first three equations of this model correspond to an Euler model with energy $E+e$, where the variable e plays the role of an internal energy. The choice of the stationary wave $e_{t}=\mathcal{O}(1)$ easily ensures the hyperbolicity of the four-equation system (5.15). Indeed, its characteristic velocities (i.e. the eigenvalues of the Jacobian matrix of the flux function) are given by:

$$
0, u, u \pm \sqrt{\frac{S}{F^{2} L(H)}+\Pi .}
$$

It would have been possible to choose another equation for $e_{t}$, but the hyperbolicity of the system would not have been guaranteed.

The structure of the homogeneous part of the system (5.15) mimics the classical Euler system with an additional stationary wave. In this context, the pressure law is

$$
\mathrm{P}(\mathrm{S}, \mathrm{U}, \mathrm{E})=2 \mathrm{E}-\mathrm{SU}^{2}+\mathrm{P}^{\mathrm{hy}}-2 \mathcal{E}=2\left(\mathrm{E}-\frac{1}{2} \mathrm{SU}^{2}\right)+\frac{1}{\mathrm{~F}^{2}} \int_{0}^{\mathrm{H}}(\mathrm{H}-3 z) \mathrm{Ld} z
$$

Remark that the non-geometrical terms of this pressure law are similar to the usual ideal gas law (5.11) of the Euler system of gas dynamics. Also, note that, by definition of $e, \Psi$ and $\Pi$, we get

$$
e=\frac{1}{2} S(\Pi-3 \Psi)=\frac{1}{2 \mathrm{U}} \int_{\Xi_{-}}^{\Xi_{+}}|\mathcal{F}| \mathrm{h}\left(v_{1}-\mathrm{u}\right)^{3} \mathrm{~d} \xi_{2}
$$

and the internal energy $e$ is nothing but the third-order moment of the velocity which was predicted in the discussion below (5.14). Finally, remark that the total energy $E+e$ has a physical meaning. Indeed, we have

$$
\begin{aligned}
E+e & =\frac{1}{2} \frac{Q^{2}}{S}+\frac{1}{2} S(\Pi-2 \Psi)+\mathcal{E} \\
& =\varepsilon+\frac{1}{2} S U^{2}+\frac{1}{2} \int_{\Xi_{-}}^{\Xi_{+}}|\mathcal{F}| \mathrm{h}\left(v_{1}-\mathrm{u}\right)^{2} \mathrm{~d} \xi_{2}+\frac{1}{2 \mathrm{U}} \int_{\Xi_{-}}^{\Xi_{+}}|\mathcal{F}| \mathrm{h}\left(v_{1}-\mathrm{u}\right)^{3} \mathrm{~d} \xi_{2} .
\end{aligned}
$$

Therefore, the total energy corresponds to the sum of the potential energy, the kinetic energy, and the quadratic and cubic errors with respect to the averaged velocity $U$.

For future reference, let us also write the system (5.15) in the set of variables $(S, Q, \Psi, \Pi)$ :

$$
\left\{\begin{array}{l}
S_{t}+Q_{\xi_{1}}=0 \\
Q_{t}+\left(\frac{Q^{2}}{S}+S \Psi\right)_{\xi_{1}}+\frac{S H_{\xi_{1}}}{F^{2}}=\frac{1}{\varepsilon} S(\mathcal{J}-\mathcal{J})+\mathcal{O}(1) \\
\left(\frac{1}{2} \frac{Q^{2}}{S}+\frac{1}{2} S \Psi\right)_{t}+\left(\frac{Q}{S}\left(\frac{1}{2} \frac{Q^{2}}{S}+\frac{1}{2} S \Pi\right)\right)_{\xi_{1}}+\frac{Q_{\xi_{1}}}{F^{2}}=\frac{1}{\varepsilon} Q(\mathcal{J}-\mathcal{J})+\mathcal{O}(\varepsilon) \\
\left(\frac{1}{2} S(\Pi-3 \Psi)\right)_{t}=\mathcal{O}(1)
\end{array}\right.
$$


At this level, the above system is hyperbolic but it does not recover the required asymptotic expansion (5.6). The goal of the next section is to calibrate the $\mathcal{O}(1)$ in order to recover (5.6).

\subsubsection{Consistency of the new model with the asymptotic expansions}

In (5.16), the zeroth-order asymptotic expansion of the discharge $Q$ is satisfied by construction of the discharge equation, see section 5.1. However, we also wish to recover its first-order asymptotic expansion, from which the energy equation of the model (5.16) is based. To that end, we necessarily need to ensure the zerothorder asymptotic expansions of $\Psi$ and $\Pi$ for the left-hand side of the energy equation to be consistent with the asymptotic regime. Therefore, the following asymptotic expansions on the discharge, enstrophy and potential have to be satisfied by the final four-equation model:

$$
\mathrm{Q}=\mathrm{Q}^{(0)}+\varepsilon \mathrm{Q}^{(1)}+\mathcal{O}\left(\varepsilon^{2}\right), \quad \Psi=\Psi^{(0)}+\mathcal{O}(\varepsilon) \quad \text { and } \quad \Pi=\Pi^{(0)}+\mathcal{O}(\varepsilon) .
$$

To write the expressions of $\Psi^{(0)}$ and $\Pi^{(0)}$ in a more compact way, we introduce the following simplified notation:

$$
\mathcal{M}_{\mathrm{n}}=\int_{\Xi_{-}}^{\Xi_{+}}|\mathcal{F}| \mathrm{h}\left(\frac{\mathrm{C}}{|\mathcal{F}|^{3 / 2}}\right)^{\mathrm{n}} \mathrm{d} \xi_{2} .
$$

Using this notation, we immediately obtain $S=\mathcal{M}_{0}$ and $Q^{(0)}=\sqrt{|\Lambda|} \operatorname{sgn}(\Lambda) \mathcal{M}_{1}$. In addition, straightforward computations yield the following asymptotic expansions for $\Psi$ and $\Pi$ :

$$
S \Psi^{(0)}=|\Lambda|\left(\mathcal{M}_{2}-\frac{\mathcal{M}_{1}^{2}}{\mathcal{M}_{0}}\right) \quad \text { and } \quad S \Pi^{(0)}=|\Lambda|\left(\frac{\mathcal{M}_{0} \mathcal{M}_{3}}{\mathcal{M}_{1}}-\frac{\mathcal{M}_{1}^{2}}{\mathcal{M}_{0}}\right) .
$$

As expected, in a U-shaped channel, these two quantities vanish. Note that, like the asymptotic expansions of $Q$, we get that $\Psi^{(0)}$ and $\Pi^{(0)}$ only depend on geometric quantities and on $\mathrm{H}$.

To ensure the asymptotic expansions (5.17), we suggest the introduction of new source terms in the model, designed to relax the enstrophy and the potential towards their respective zeroth-order expansions. These source terms cannot be added to the energy equation, since its current form is necessary to recover the first-order approximation of the discharge.

In addition, the new relaxation source terms shall have to be independent of $\Lambda$, since $\Lambda$ contains $H_{\xi_{1}}$. Therefore, the naive choice which consists in simply adding a source term under the form $\frac{1}{\varepsilon}\left(\Psi^{(0)}-\Psi\right)$ is not advisable, since $\Psi^{(0)}$, given by (5.18), contains $\Lambda$. This remark also holds for $\Pi^{(0)}$. To address this issue, we mimic the way the friction term $\mathcal{J}$ was defined to introduce $\mathcal{J}_{\psi}$ and $\mathcal{J}_{\Pi}$, given by:

$$
\mathcal{J}_{\Psi}=\Lambda \frac{\Psi}{\Psi(0)}=\operatorname{sgn}(\mathrm{Q})|\Lambda| \frac{\Psi}{\Psi^{(0)}} \quad \text { and } \quad \mathcal{J}_{\Pi}=\Lambda \frac{\Pi}{\Pi^{(0)}}=\operatorname{sgn}(\mathrm{Q})|\Lambda| \frac{\Pi}{\Pi^{(0)}} .
$$

According to the definitions (5.18), $\mathcal{J}_{\Psi}$ and $\mathcal{J}_{\Pi}$ are indeed independent of $\Lambda$. In addition, we have replaced $\operatorname{sgn}(\Lambda)$ with $\operatorname{sgn}(Q) \operatorname{since} \operatorname{sgn}(\Lambda)=\operatorname{sgn}\left(Q^{(0)}\right)=\operatorname{sgn}(Q)$ for small enough $\varepsilon$.

Then, we add the following relaxation term to the discharge equation:

$$
\frac{1}{\varepsilon} S K_{1} \wedge\left(1-\frac{\Psi}{\Psi(0)}\right)=\frac{1}{\varepsilon} S K_{1}\left(\Lambda-\mathcal{J}_{\Psi}\right)=\frac{1}{\varepsilon} S K_{1}\left(\mathcal{J}-\partial_{\Psi}\right)-K_{1} \frac{S H_{\xi_{1}}}{F^{2}},
$$

where $K_{1}$ is a nonzero function of $S, Q, \Psi$ and $\Pi$, to be determined. This term ensures that $\Psi$ relaxes towards $\Psi^{(0)}$ when $\varepsilon$ goes to 0 , whatever the value of $K_{1}$, but it adds a differential term in $\mathrm{H}_{\xi_{1}}$.

We now have to relax $\Pi$ towards $\Pi^{(0)}$ when $\varepsilon$ goes to 0 , using only the fourth equation of the system. To avoid adding another differential term in $\mathrm{H}_{\xi_{1}}$ and to retain the stationary wave, we add the following relaxation term to the fourth equation:

$$
\frac{1}{\varepsilon} \mathrm{QK}_{2} \wedge\left(\frac{\Psi}{\Psi(0)}-\frac{\Pi}{\Pi^{(0)}}\right)=\frac{1}{\varepsilon} \mathrm{QK}_{2}\left(\mathcal{J}_{\Psi}-\mathcal{J}_{\pi}\right),
$$


where $K_{2}$ is another nonzero function of $S, Q, \Psi$ and $\Pi$, to be determined. Let us emphasize that, for all $K_{1} \neq 0$ and $K_{2} \neq 0$, the additional terms (5.19) and (5.20) respectively relax $\Psi$ towards $\Psi^{(0)}$ and $\Pi$ towards $\Pi^{(0)}$.

We thus modify (5.16) as follows:

$$
\left\{\begin{array}{l}
\mathrm{S}_{\mathrm{t}}+\mathrm{Q}_{\xi_{1}}=0, \\
\mathrm{Q}_{\mathrm{t}}+\left(\frac{\mathrm{Q}^{2}}{\mathrm{~S}}+\mathrm{S \Psi}\right)_{\xi_{1}}+\left(1+\mathrm{K}_{1}\right) \frac{\mathrm{SH}_{\xi_{1}}}{\mathrm{~F}^{2}}=\frac{1}{\varepsilon} \mathrm{S}\left(\mathcal{J}-\mathcal{J}+\mathrm{K}_{1}\left(\mathcal{J}-\mathcal{J}_{\Psi}\right)\right), \\
\left(\frac{1}{2} \frac{\mathrm{Q}^{2}}{\mathrm{~S}}+\frac{1}{2} \mathrm{~S} \Psi\right)_{\mathrm{t}}+\left(\frac{\mathrm{Q}}{\mathrm{S}}\left(\frac{1}{2} \frac{\mathrm{Q}^{2}}{\mathrm{~S}}+\frac{1}{2} \mathrm{~S} \Pi\right)\right)_{\xi_{1}}+\frac{\mathrm{QH}_{\xi_{1}}}{\mathrm{~F}^{2}}=\frac{1}{\varepsilon} \mathrm{Q}(\mathcal{J}-\mathcal{J}), \\
\left(\frac{1}{2} \mathrm{~S}(\Pi-3 \Psi)\right)_{\mathrm{t}}=\frac{1}{\varepsilon} \mathrm{QK}_{2}\left(\mathcal{J} \Psi-\mathcal{J}_{\Pi}\right) .
\end{array}\right.
$$

Note that we have added relaxation source terms in $\mathcal{O}(1)$ in the discharge and internal energy equations, which is consistent with what was prescribed in (5.16). Since (5.21) is the final model, up to the choice of $K_{1}$ and $K_{2}$, we have now removed the $\mathcal{O}(1)$ and $\mathcal{O}(\varepsilon)$. The above model is equivalent to the following one, obtained by introducing $\wedge$ back in the system:

$$
\left\{\begin{array}{l}
\mathrm{S}_{\mathrm{t}}+\mathrm{Q}_{\xi_{1}}=0, \\
\mathrm{Q}_{\mathrm{t}}+\left(\frac{\mathrm{Q}^{2}}{\mathrm{~S}}+\mathrm{S \Psi}\right)_{\xi_{1}}=\frac{1}{\varepsilon} \mathrm{S}\left(\Lambda-\mathcal{J}+\mathrm{K}_{1}\left(\Lambda-\partial_{\Psi}\right)\right), \\
\left(\frac{1}{2} \frac{\mathrm{Q}^{2}}{\mathrm{~S}}+\frac{1}{2} \mathrm{~S} \Psi\right)_{\mathrm{t}}+\left(\frac{\mathrm{Q}}{\mathrm{S}}\left(\frac{1}{2} \frac{\mathrm{Q}^{2}}{\mathrm{~S}}+\frac{1}{2} \mathrm{~S} \Pi\right)\right)_{\xi_{1}}=\frac{1}{\varepsilon} \mathrm{Q}(\Lambda-\mathcal{J}), \\
\left(\frac{1}{2} \mathrm{~S}(\Pi-3 \Psi)\right)_{\mathrm{t}}=\frac{1}{\varepsilon} \mathrm{QK}_{2}\left(\partial_{\Psi}-\partial_{\Pi}\right) .
\end{array}\right.
$$

To summarize, from the zeroth-order asymptotic expansion of the energy equation (5.22c), we immediately recover $\Lambda=\mathcal{J}$, and thus $\mathrm{Q}=\mathrm{Q}^{(0)}$. Then, from the zeroth-order asymptotic expansion of the discharge equation (5.22b), we get $\Lambda=J_{\Psi}$, i.e. $\Psi=\Psi^{(0)}$. Afterwards, the zeroth-order expansion of the internal energy equation (5.22d) ensures that $\Lambda=J_{\Pi}$, which yields $\Pi=\Pi^{(0)}$. Thus, the three required zeroth-order asymptotic expansions are recovered. In addition, it turns out that the first-order expansion of the energy equation (5.22c) instantly recovers the correct formula for $\mathrm{Q}^{(1)}$. Therefore, the model (5.22) (or, equivalently, (5.21)) ensures the required asymptotic expansions (5.17), whatever the choice of $K_{1}$ and $K_{2}$.

\subsection{Mathematical properties of the model; comparison with the classical shallow water system}

Equipped with the model (5.21) (or equivalently (5.22)), we are now able to check its mathematical properties, as well as perform a theoretical comparison with the classical shallow water system. In addition, we need to fix the values of the - so far - arbitrary functions $K_{1}$ and $K_{2}$.

We first note that, unfortunately, the addition of the term $\mathrm{K}_{1} \mathrm{SH}_{\xi_{1}} / \mathrm{F}^{2}$ in (5.21b) prevents us from rewriting the system under an Euler-like form, since the pressure in (5.21b) would be different from the one in (5.21c). However, we are able to use the almost-Euler-like underlying structure to our advantage in the next developments.

To simplify the forthcoming analysis, we note that $S_{\xi_{1}}=L(H) H_{\xi_{1}}$ to rewrite the system (5.21) under the non-conservative form $W_{t}+A(W) W_{\xi_{1}}=R(W)$, with $W=(S, U, S \Psi, S \Pi)^{T}$, and where the flux matrix $A(W)$ and 
source terms vector $R(W)$ are given by

$$
A(W)=\left(\begin{array}{cccc}
U & S & 0 & 0 \\
\frac{1+K_{1}}{L(H) F^{2}} & U & \frac{1}{S} & 0 \\
\frac{-2 S U K_{1}}{L(H) F^{2}} & S \Pi & -2 U & U \\
\frac{-6 S U K_{1}}{L(H) F^{2}} & 3 S \Pi & -6 U & 3 U
\end{array}\right) \text { and } R(W)=\frac{1}{\varepsilon}\left(\begin{array}{c}
0 \\
\mathcal{J}-\mathcal{J}+K_{1}\left(\mathcal{J}-\mathcal{J}_{\Psi}\right) \\
-2 S U K_{1}\left(\mathcal{J}-\mathcal{J}_{\Psi}\right) \\
2 S U\left[K_{2}\left(\mathcal{J}_{\Psi}-\mathcal{J}_{\Pi}\right)-3 K_{1}\left(\mathcal{J}-\mathcal{J}_{\Psi}\right)\right]
\end{array}\right) .
$$

\subsubsection{Hyperbolicity and algebraic properties}

For comparison purposes, recall that the characteristic velocities of the classical shallow water system in $(S, Q)$ variables are

$$
\mathrm{U} \pm \sqrt{\frac{\mathrm{S}}{\mathrm{F}^{2} \mathrm{~L}(\mathrm{H})}} .
$$

In addition, the two characteristic fields associated to these characteristic velocities are genuinely nonlinear.

As stated in section 5.2.2, before adding the relaxation source term for $\Psi$, i.e. for $K_{1}=0$, the characteristic velocities of the four-equation model were

$$
0, \lambda_{\mathrm{U}}=\mathrm{U}, \lambda_{ \pm}=\mathrm{U} \pm \sqrt{\frac{\mathrm{S}}{\mathrm{F}^{2} \mathrm{~L}(\mathrm{H})}+\Pi} .
$$

The characteristic fields associated to 0 and $\mathrm{U}$ were linearly degenerate, while the other two characteristic fields were genuinely nonlinear. Straightforward computations show that this system was hyperbolic as soon as $S \neq 0$, and strictly hyperbolic as soon as $S \neq 0$ and $U \neq 0$.

Now, because of the relaxation terms, the characteristic velocities of the system (5.21), i.e. the eigenvalues of the matrix $A(W)$, can no longer be written under a tractable form. Thanks to (5.21d), 0 is still a characteristic velocity of this system. In order to get a better idea of the other three eigenvalues, we perform an asymptotic expansion with respect to the small Froude number $F$. Let us denote by $\chi_{A(W)}(\lambda)$ the characteristic polynomial of the matrix $A(W)$. After tedious but straightforward computations, we prove that the following asymptotic expansions of $\lambda$ ensure that $\chi_{\mathrm{A}(W)}(\lambda)=\mathcal{O}\left(\mathrm{F}^{2}\right)$ :

$$
\begin{aligned}
& \lambda_{\mathrm{U}}^{\mathrm{K}_{1}}=\mathrm{U} \frac{1+3 \mathrm{~K}_{1}}{1+\mathrm{K}_{1}}+\mathcal{O}\left(\mathrm{F}^{2}\right) \\
& \lambda_{ \pm}^{\mathrm{K}_{1}}=\frac{\mathrm{U}}{1+\mathrm{K}_{1}} \pm \sqrt{\frac{\mathrm{S}}{\mathrm{L}(\mathrm{H})}}\left(\frac{\sqrt{1+\mathrm{K}_{1}}}{\mathrm{~F}}+\frac{\mathrm{F}}{2} \frac{\Pi+\mathrm{K}_{1}\left(2 \Pi+\mathrm{K}_{1}\left(\Pi-3 \mathrm{U}^{2}\right)\right)}{\left(1+\mathrm{K}_{1}\right)^{5 / 2}}\right)+\mathcal{O}\left(\mathrm{F}^{2}\right) .
\end{aligned}
$$

Note that, if $K_{1}=0$, we recover the characteristic velocities $\lambda_{u}$ and $\lambda_{ \pm}$. In addition, for the system to be hyperbolic, these eigenvalues have to take real values. Therefore, for small enough $F$, a necessary condition for the hyperbolicity is $1+K_{1}>0$, and we need to determine $K_{1}$ with this constraint in mind.

\subsubsection{Linear stability}

Let us proceed with determining suitable $\mathrm{K}_{1}$ and $\mathrm{K}_{2}$. To that end, we perform a linear stability analysis of the system (5.22), detailed in Appendix B. We obtain the following expressions:

$$
\mathrm{K}_{1}=-\frac{S \Psi_{0}}{S U_{0}^{2}}=-\frac{S^{2} \Psi^{(0)}}{\left(Q^{(0)}\right)^{2}} \quad \text { and } \quad K_{2}=\frac{S \Pi_{0}}{S U_{0}^{2}}=\frac{S^{2} \Pi^{(0)}}{\left(Q^{(0)}\right)^{2}}
$$


As a conclusion, the final hyperbolic four-equation model prescribed by this linear stability analysis is:

$$
\left\{\begin{array}{l}
S_{\mathrm{t}}+\mathrm{Q}_{\xi_{1}}=0, \\
\mathrm{Q}_{\mathrm{t}}+\left(\frac{\mathrm{Q}^{2}}{\mathrm{~S}}+\mathrm{S \Psi}\right)_{\xi_{1}}+\left(1-\frac{\mathrm{S}^{2} \Psi(0)}{\left(\mathrm{Q}^{(0)}\right)^{2}}\right) \frac{S H_{\xi_{1}}}{\mathrm{~F}^{2}}=\frac{1}{\varepsilon} S\left(\mathcal{J}-\mathcal{J}-\frac{S^{2} \Psi(0)}{\left(\mathrm{Q}^{(0)}\right)^{2}}\left(\mathcal{J}-\mathcal{J}_{\Psi}\right)\right) \\
\left(\frac{1}{2} \frac{\mathrm{Q}^{2}}{\mathrm{~S}}+\frac{1}{2} S \Psi\right)_{\mathrm{t}}+\left(\frac{\mathrm{Q}}{\mathrm{S}}\left(\frac{1}{2} \frac{\mathrm{Q}^{2}}{\mathrm{~S}}+\frac{1}{2} S \Pi\right)\right)_{\xi_{1}}+\frac{\mathrm{QH}_{\xi_{1}}}{\mathrm{~F}^{2}}=\frac{1}{\varepsilon} \mathrm{Q}(\mathcal{J}-\mathcal{J}) \\
\left(\frac{1}{2} \mathrm{~S}(\Pi-3 \Psi)\right)_{\mathrm{t}}=\frac{1}{\varepsilon} \mathrm{Q} \frac{S^{2} \Pi^{(0)}}{\left(\mathrm{Q}^{(0)}\right)^{2}}\left(\mathcal{J}_{\Psi}-\mathcal{J}_{\Pi}\right)
\end{array}\right.
$$

Regarding the hyperbolicity of this system, recall that it is hyperbolic under the condition $1+\mathrm{K}_{1}>0$ for small enough $F$. With $K_{1}$ given by (5.23), this condition becomes $S \Psi^{(0)}<\left(Q^{(0)}\right)^{2} / S$. Firstly, note that for a U-shaped channel with uniform friction, $S \Psi^{(0)}$ vanishes and the hyperbolicity condition is always satisfied. Secondly, according to the definition (5.13) of $S \Psi$, this hyperbolicity condition is equivalent to

$$
\int_{\Xi_{-}}^{\Xi_{+}}|\mathcal{F}| \mathrm{h}\left(\mathrm{u}^{(0)}\right)^{2} \mathrm{~d} \xi_{2}<2 \mathrm{~S}\left(\mathrm{u}^{(0)}\right)^{2}
$$

where $U^{(0)}=Q^{(0)} / S$. Since $2 S\left(U^{(0)}\right)^{2}$ is two times an average of $h\left(u^{(0)}\right)^{2}$, this condition should be satisfied in the regimes under consideration. Indeed, multiple tests run by the authors, in many geometries and for many friction laws, have all shown that this condition is easily satisfied. As a consequence, the system (5.24) is hyperbolic.

\section{Validation of the four-equation model}

The four-equation model (5.24) has been built in order to possess the required consistency properties. This last section is devoted to the numerical validation of our four-equation model. In its first part, we present some reference solutions, as well as the numerical schemes we use to approximate these solutions. The second part of this section is then dedicated to the numerical experiments themselves.

In this section, we will consider the three 1D models at our disposal:

- the classical shallow water model, later referred to as SW, given by the dimensionless version of (1.2) with $\beta=1$, which is consistent only when considering U-shaped channels;

- the shallow water model equipped with the friction term (5.3), later referred to as A0, consistent with the zeroth-order asymptotic expansion of $\mathrm{Q}$ and summarized in section 7.1.1;

- the four-equation model (5.24), later referred to as A1, consistent with the first-order asymptotic expansion of $\mathrm{Q}$ and summarized in section 7.1.2.

The goal of this section is to compare the results of the three models under consideration. We expect the SW model to be inconsistent as soon as the channel is not U-shaped. Moreover, we also expect that the A1 model should yield more accurate results than the A0 one.

\subsection{Reference solutions and numerical considerations}

We begin by discussing the theory of the backwater curves (i.e. the stationary solutions) of the three 1D models at our disposal. Then, we introduce the manufactured stationary 2D reference solution that will be used to perform several stationary numerical experiments. Lastly, we present the numerical discretization used throughout the experiments. 


\subsubsection{Backwater curves in $1 D$}

We first consider the backwater curves of the 1D models under consideration (i.e. its stationary solutions, free from time). For the classical shallow water system in $(S, Q)$ variables (i.e. the dimensionless version of (1.2)), they are given by a constant discharge $\mathrm{Q}=\mathrm{Q}_{0}$ and the profile equation

$$
\mathrm{H}_{\xi_{1}}=\frac{\mathrm{J}_{0}}{\delta} \frac{\mathcal{J}-\mathrm{J}_{\mathrm{SW}}}{1-\mathrm{F}^{2} \mathrm{~L}(\mathrm{H}) \frac{\mathrm{Q}_{0}^{2}}{\mathrm{~S}^{3}}},
$$

where the friction model $\mathcal{J}_{s} w$ is given by

$$
\partial_{\mathrm{sW}}=\frac{\mathrm{Q}_{0}\left|\mathrm{Q}_{0}\right|}{\mathrm{H}^{2+\mathrm{p}_{\mathrm{c}} \mathrm{c}_{\mathrm{SW}}^{2}}}
$$

As $\delta / J_{0}$ goes to 0 , the backwater curves become driven only by the numerator of the $\mathrm{H}_{\xi_{1}}$ equation. More specifically, $\mathrm{H}$ will be equal to $\mathrm{H}_{\mathrm{n}}^{S W}$, defined as the value of $\mathrm{H}$ canceling the numerator $\mathcal{J}-\mathcal{J}_{s} w$ for a uniform flow. This quantity $\mathrm{H}_{\mathrm{n}}$ is referred to as the normal height in the hydraulic literature. Thus, we get

$$
\mathrm{H}_{\mathrm{n}}^{\mathrm{SW}}=\left(\frac{\mathrm{Q}_{0}\left|\mathrm{Q}_{0}\right|}{\mathcal{J} \mathrm{c}_{\mathrm{SW}}^{2}}\right)^{\frac{1}{2+\mathrm{p}}} .
$$

For the A0 model, the only difference is the friction model, and the backwater equation reads

$$
\mathrm{H}_{\xi_{1}}=\frac{\mathrm{J}_{0}}{\delta} \frac{\mathcal{J}-\mathcal{J}}{1-\mathrm{F}^{2} \mathrm{~L}(\mathrm{H}) \frac{\mathrm{Q}_{0}^{2}}{\mathrm{~S}^{3}}},
$$

instead of (6.1). Therefore, we note that the value of the normal height depends on the friction model $\mathcal{J}$ under consideration, given by (5.5): J depends on $S$, and thus on $\mathrm{H}$. The normal height $\mathrm{H}_{\mathrm{n}}^{\mathrm{AO}}$ is now implicitly defined as the value of $\mathrm{H}$ canceling $\mathcal{J}-\mathcal{J}$.

The backwater curves of the four-equation model (5.24), in addition to the constant discharge $Q=Q_{0}$, read as follows:

$$
\left\{\begin{array}{l}
H_{\xi_{1}}=\frac{J_{0}}{\delta} \frac{\mathcal{J}-J-\frac{F^{2}}{2 S}(S \Pi)_{\xi_{1}}}{1-F^{2} L(H)\left(\frac{Q_{0}^{2}}{S^{3}}+\frac{S \Pi}{2 S^{2}}\right)} \\
(S \Psi)_{\xi_{1}}=\frac{1}{\varepsilon} S\left(J-J-\frac{S \Psi^{(0)}}{\left(Q^{(0)}\right)^{2}}(\mathcal{J}-\mathcal{J} \Psi)\right)-\left(1-\frac{S \Psi^{(0)}}{\left(Q^{(0)}\right)^{2}}-F^{2} L(H) \frac{Q_{0}^{2}}{S^{3}}\right) \frac{S H_{\xi_{1}}}{F^{2}} \\
\frac{\Psi}{\Psi(0)}=\frac{\Pi}{\Pi^{(0)}} .
\end{array}\right.
$$

We note that the normal height $\mathrm{H}_{n}^{\mathrm{A} 1}$ associated to the four-equation model is also defined by $\mathcal{J}-\mathcal{J}=0$. However, the backwater curve for the four-equation model contains an additional $\mathcal{O}\left(\mathrm{F}^{2}\right)$ term compared to the one of the shallow water model. This additional term comes from the fact that we ensure a first-order approximation on the discharge.

\subsubsection{Steady $2 D$ reference solution}

In the forthcoming developments, we consider a stationary solution of the 2D equations (2.4) in the weakly meandering asymptotic regime, characterized by $R_{y}=\mathcal{O}(\varepsilon), R_{l}=\mathcal{O}(\varepsilon)$ and $\varepsilon \ll 1$. The goal of these computations is to rewrite this system under a computationally tractable form. Thanks to this new form, we will be able to manufacture a steady reference solution, against which the three 1D models will be tested. 
Let us begin by writing the unsteady system (2.4) in the asymptotic regime under consideration. Assuming a stationary solution and a weakly meandering river, (2.4) becomes:

$$
\left\{\begin{array}{l}
\left(|\mathcal{F}| h v_{1}\right)_{\xi_{1}}+\left(|\mathcal{F}| h v_{2}\right)_{\xi_{2}}=0, \\
v_{1}\left(v_{1}\right)_{\xi_{1}}+v_{2}\left(v_{1}\right)_{\xi_{2}}+\frac{1}{|\mathcal{F}|^{2} F^{2}}\left(h_{\xi_{1}}+\phi_{\xi_{1}}\right)=\frac{1}{\varepsilon}\left(\frac{\mathcal{J}}{|\mathcal{F}|^{2}}-\frac{|\mathcal{F}| v_{1}\left|v_{1}\right|}{\mathrm{C}^{2}}\right)+\mathcal{O}(\varepsilon), \\
h_{\xi_{2}}+\phi_{\xi_{2}}=\mathcal{O}\left(\varepsilon^{2}\right) .
\end{array}\right.
$$

The first step to solve this system is to note that (6.5a) imposes that the discharge be divergence-free. Therefore, we introduce a discharge potential $\Phi$, such that $|\mathcal{F}| \mathrm{h} \nu_{1}=\Phi_{\xi_{1}}$ and $|\mathcal{F}| h v_{2}=\Phi_{\xi_{2}}$. In this context, the equation (6.5a) is equivalent to $\Delta \Phi=0$. We prescribe suitable boundary conditions, ensuring that $|\mathcal{F}| \mathrm{h} v_{2}=\mathcal{O}(\varepsilon)$ to satisfy the asymptotic regime. Numerically solving this first equation gives the quantities $|\mathcal{F}| \mathrm{h} v_{1}$ and $|\mathcal{F}| \mathrm{h} v_{2}$.

Now, we need to compute $h\left(\xi_{1}, \xi_{2}\right)$. Since $|\mathcal{F}| h v_{2}=\mathcal{O}(\varepsilon)$, (6.5b) becomes

$$
\frac{|\mathcal{F}| v_{1}\left|v_{1}\right|}{C^{2}}=\frac{\Lambda}{|\mathcal{F}|^{2}}-\varepsilon v_{1}\left(v_{1}\right)_{\xi_{1}}+\mathcal{O}\left(\varepsilon^{2}\right)
$$

We assume that the friction law is the usual Chézy one, given in dimensionless variables by $\mathrm{C}^{2}=\mathrm{c}^{2} \mathrm{~h}^{\mathrm{p}}$. We look for a non-uniform Chézy coefficient $c\left(\xi_{1}, \xi_{2}\right)$, which therefore becomes an unknown of the problem. At this level, we have two unknowns, $h\left(\xi_{1}, \xi_{2}\right)$ and $c\left(\xi_{1}, \xi_{2}\right)$.

To address this issue, let us use both the divergence-free discharge and the asymptotic regime. First, we get from (6.6) the equation on $c$, as follows:

$$
c^{2}=\frac{|\mathfrak{F}|^{2} q|q| h^{1-p}}{|\mathcal{F}| \Lambda h^{3}-\varepsilon q\left(|\mathcal{F}| h q_{\xi_{1}}-q|\mathcal{F}| h_{\xi_{1}}\right)}+\mathcal{O}\left(\varepsilon^{2}\right),
$$

where the already computed quantity $|\mathcal{F}| h v_{1}$ is denoted by $q$. Then, we replace the velocities in (6.6) by their asymptotic expansions. Therefore, the equation (6.6) becomes

$$
\varepsilon\left(v_{1}^{(0)}\left(v_{1}^{(0)}\right)_{\xi_{1}}+\frac{2|\mathcal{F}| v_{1}^{(1)}\left|v_{1}^{(0)}\right|}{(1+c)^{2} h^{p}}\right)=\frac{\Lambda}{|\mathcal{F}|^{2}}-\frac{|\mathcal{F}| v_{1}^{(0)}\left|v_{1}^{(0)}\right|}{c^{2} h^{p}}+\mathcal{O}\left(\varepsilon^{2}\right),
$$

where the asymptotic expansions $v_{1}^{(0)}$ and $v_{1}^{(1)}$ are respectively given by (3.7) and (3.10).

Injecting (6.7) into (6.8) therefore yields an equation whose only unknown is $h$, and which manufactures the Chézy coefficient such that $h+\phi$ is almost uniform in space. Solving this equation for $h$ allows us to plug this value into (6.7), and therefore to complete the determination of the $2 \mathrm{D}$ reference stationary solution, while ensuring that the correct asymptotic regime is satisfied. These computations are made easier by the fact that we have $h_{\xi_{2}}+\phi_{\xi_{2}}=0$.

This process, in addition to the usual reference scaling, requires to fix the values of $|\mathcal{F}| h v_{1}$ and $|\mathcal{F}| h v_{2}$ at the boundaries, as well as an equivalent to the 1D normal height discussed in section 6.1.1. These three dimensionless quantities will respectively be denoted by $|\mathcal{F}| \mathrm{h}\left(v_{1}\right)_{0},|\mathcal{F}| \mathrm{h}\left(v_{2}\right)_{0}$, and $\mathrm{H}_{\mathrm{n}}^{2 \mathrm{D}}$, and numerical values will be given in the relevant section. In the end, we get a solution whose free surface $\mathrm{H}=\mathrm{h}+\phi$ will be close to the fixed normal height.

\subsubsection{Discretization}

We now briefly discuss the numerical discretization of the models under consideration, to be used in the validation numerical experiments of the next part of this section. 
The steady $2 \mathrm{D}$ reference solution. Obtaining this $2 \mathrm{D}$ reference solution relies on a natural discretization of the set of equations presented in section 6.1.2. For the sake of simplicity, we use a finite difference method on a Cartesian grid with $n_{\xi_{1}}$ points in the $\xi_{1}$-direction and $n_{\xi_{2}}$ points in the $\xi_{2}$-direction.

The backwater curves. Since the parameter $\delta / \mathrm{J}_{0}$ can become small, stiff terms can appear in the backwater curves given by (6.1), (6.3) and (6.4). Therefore, we adopt a fully implicit finite difference discretization of the three models under consideration. We use a uniform 1D mesh with $n_{\xi_{1}}$ discretization elements. In addition, for the Chézy coefficient and the topography, an underlying 2D mesh is needed. To address this issue, we use the mesh presented above, with $n_{\xi_{1}} \times n_{\xi_{2}}$ points.

The unsteady problem in 1D. Numerical experiments with steady solutions will have convinced us that the SW model is unsuitable as soon as the channel is no longer U-shaped. Therefore, we only consider the A0 and A1 models for 1D unsteady problems. These models are governed by hyperbolic systems of balance laws. In addition, some terms in $\mathcal{O}\left(\frac{1}{\varepsilon}\right)$ and $\mathcal{O}\left(\frac{1}{\mathrm{~F}^{2}}\right)$ are stiff. These two remarks prompt us to propose a finite difference splitting strategy, where the non-stiff part is treated explicitly, and the stiff part is treated implicitly. This amounts to introducing IMEX strategies that are both asymptotic preserving (regarding stiff source terms) and adapted to Low Froude number limits: see e.g. [3] and references therein for more details.

For the A0 model, we denote by $\left(S_{A O}\right)_{i}^{n}$ and $\left(Q_{A O}\right)_{i}^{n}$ the discretizations of the section and the discharge at the spatial node $\left(\xi_{1}\right)_{i}$ and at time $t^{n}$. The first step of the space-time discretization consists in considering the non-stiff part of the A0 system, given by:

$$
\left\{\begin{array}{l}
S_{t}+Q_{\xi_{1}}=0 \\
Q_{t}+\left(\frac{Q^{2}}{S}\right)_{\xi_{1}}=0
\end{array}\right.
$$

This non-stiff part is discretized explicitly using an upwind flux, as follows:

$$
\left\{\begin{array}{l}
\left(S_{A O}\right)_{i}^{n+\frac{1}{2}}=\left(S_{A O}\right)_{i}^{n}-\frac{\Delta t}{\Delta x}\left(\left(Q_{A O}\right)_{i+\frac{1}{2}}^{n}-\left(Q_{A O}\right)_{i-\frac{1}{2}}^{n}\right), \\
\left(Q_{A O}\right)_{i}^{n+\frac{1}{2}}=\left(Q_{A O}\right)_{i}^{n}-\frac{\Delta t}{\Delta x}\left(\left(\frac{Q_{A O}^{2}}{S_{A O}}\right)_{i+\frac{1}{2}}^{n}-\left(\frac{Q_{A O}^{2}}{S_{A O}}\right)_{i-\frac{1}{2}}^{n}\right),
\end{array}\right.
$$

where the upwind fluxes are given, for any quantity $X$, by

$$
X_{i+\frac{1}{2}}^{n}= \begin{cases}X_{i}^{n} & \text { if } \frac{1}{2}\left(\left(Q_{A O}\right)_{i}^{n}+\left(Q_{A O}\right)_{i+1}^{n}\right)>0, \\ X_{i+1}^{n} & \text { otherwise, }\end{cases}
$$

where $\Delta \mathrm{x}$ is the space step, and where $\Delta \mathrm{t}$ is the time step, related to $\Delta \mathrm{x}$ through a usual CFL-like condition (see e.g. [19]), and such that $\Delta t=\mathcal{O}(\Delta x)$. The intermediate values $\left(S_{A O}\right)_{i}^{n+\frac{1}{2}}$ and $\left(Q_{A O}\right)_{i}^{n+\frac{1}{2}}$ are then used as initial conditions for the stiff part of the A0 system, given by:

$$
\left\{\begin{array}{l}
\mathrm{S}_{\mathrm{t}}=0, \\
\mathrm{Q}_{\mathrm{t}}+\frac{\mathrm{SH}_{\xi_{1}}}{\mathrm{~F}^{2}}=\frac{1}{\varepsilon} \mathrm{S}\left(\mathcal{J}-\Lambda \frac{\mathrm{Q}|\mathrm{Q}|}{\mathrm{Q}^{(0)}\left|\mathrm{Q}^{(0)}\right|}\right) .
\end{array}\right.
$$

We immediately note that $S$, and therefore $H$, is time-independent in this stiff second step. Therefore, we obtain $\left(S_{A O}\right)_{i}^{n+1}=\left(S_{A O}\right)_{i}^{n+\frac{1}{2}}$. Recalling the friction model (5.3) and noting that $\operatorname{sgn}(Q)=\operatorname{sgn}\left(Q^{(0)}\right)$ for small 
enough $\varepsilon$, the updated discharge is then given by the ODE

$$
\mathrm{Q}_{\mathrm{t}}=\frac{1}{\varepsilon} \mathrm{S} \wedge\left(1-\frac{\mathrm{Q}^{2}}{\left(\mathrm{Q}^{(0)}\right)^{2}}\right)
$$

whose only unknown is $\mathrm{Q}$. Integrating this ODE between times 0 and $t$, we get the following exact expression for Q:

$$
\mathrm{Q}(\mathrm{t})=\mathrm{Q}^{(0)} \frac{\tanh \left(\frac{1}{\varepsilon} \frac{\mathrm{S}|\Lambda|}{\left|\mathrm{Q}^{(0)}\right|} \mathrm{t}\right)+\frac{\mathrm{Q}(0)}{\mathrm{Q}^{(0)}}}{1+\tanh \left(\frac{1}{\varepsilon} \frac{\mathrm{S}|\Lambda|}{\left|\mathrm{Q}^{(0)}\right|} \mathrm{t}\right) \frac{\mathrm{Q}(0)}{\mathrm{Q}^{(0)}}} .
$$

The corresponding discretization reads as follows:

$$
\left(Q_{A O}\right)_{i}^{n+1}=\left(Q^{(0)}\right)_{i}^{n+1} \frac{\tanh \left(\frac{1}{\varepsilon} \frac{\left(S_{A O}\right)_{i}^{n+1}(|\Lambda|)_{i}^{n+1}}{\left|\left(Q^{(0)}\right)_{i}^{n+1}\right|} \Delta t\right)+\frac{\left(Q_{A O}\right)_{i}^{n+\frac{1}{2}}}{\left(Q^{(0)}\right)_{i}^{n+1}}}{1+\tanh \left(\frac{1}{\varepsilon} \frac{\left(S_{A O}\right)_{i}^{n+1}(|\Lambda|)_{i}^{n+1}}{\left|\left(Q^{(0)}\right)_{i}^{n+1}\right|} \Delta t\right) \frac{\left(Q_{A O}\right)_{i}^{n+\frac{1}{2}}}{\left(Q^{(0)}\right)_{i}^{n+1}}}
$$

where we recall that $\Lambda$ and $Q^{(0)}$ only depend on $S_{A 0}$ and the node $i$, and therefore we note that the above equation explicitly determines $\left(Q_{A O}\right)_{i}^{n+1}$. In addition, note that we correctly recover $\left(Q_{A 0}\right)_{i}^{n+1} \rightarrow\left(Q^{(0)}\right)_{i}^{n+1}$ when $\varepsilon \rightarrow 0$. The presentation of the numerical discretization of the model A0 is thus complete.

For the A1 model, we apply a similar procedure, which we do not write in detail for the sake of conciseness. The main difference between the two discretizations is that the system of ODEs resulting from the stiff part can no longer be exactly solved, and a multivariate nonlinear root-finding algorithm (in practice, Newton's method) has to be applied. In addition, thanks to the splitting procedure, we also correctly recover the discrete first-order asymptotic regime.

The unsteady model in 2D. Finally, to compare unsteady $1 \mathrm{D}$ results to a reference solution, we require a numerical scheme for the 2D system (2.3). We elect to use the DASSFLOW code $[8,24]$, designed to solve this system in conservative form and validated on multiple $2 \mathrm{D}$ test cases.

\subsection{Numerical validation}

The second part of this section is devoted to the numerical validation itself. We begin by discussing the chosen geometries and the values we give to the reference dimensionalization parameters. Then, we compare the steady solutions of each 1D model to the stationary 2D reference solution, as well as the unsteady solutions of the 1D models.

\subsubsection{Geometry and values of the dimensionalization parameters}

For the sake of simplicity, we consider a non-meandering, evenly sloped and symmetrical trapezoidal channel. In addition, to avoid treating dry areas in the discretization, we suppose that the channel is walled and filled with water. The dimensionalization parameters are chosen such that the channel roughly corresponds to the Garonne river upstream of the city of Toulouse. 
Geometry. Recall that the topography is given by $Z\left(\xi_{1}, \xi_{2}\right)=b_{0}\left(\xi_{1}\right)+\frac{\delta}{I_{0}} \phi\left(\xi_{1}, \xi_{2}\right)$. Also, note that $b_{0}\left(\xi_{1}\right)$ only intervenes through its derivative $\left(b_{0}\right)_{\xi_{1}}$, which we take equal to 1 . Furthermore, we assume that the computational domain is $\left[0,\left(\xi_{1}\right)_{+}\right]$, whose numerical value is given later. For simplicity, we assume that the longitudinal slope is only driven by $b_{0}$, and we take $\phi\left(\xi_{1}, \xi_{2}\right)=\phi\left(\xi_{2}\right)$. Then, we get a symmetrical trapezoidal channel by considering

$$
\phi\left(\xi_{2}\right)= \begin{cases}\left(-\xi_{2}-l\right) \tan \theta & \text { if } \Xi_{-}<\xi_{2} \leqslant-l, \\ 0 & \text { if }-l \leqslant \xi_{2} \leqslant l, \\ \left(\xi_{2}-l\right) \tan \theta & \text { if } l \leqslant \xi_{2}<\Xi_{+},\end{cases}
$$

where the notations are explained in figure 3 , and where we take $\Xi_{-}=-\Xi_{+}$. The numerical values of $\Xi_{ \pm}, l$ and $\theta$ are given in the next paragraph, for each experiment. Note that a U-shaped channel is a specific case of this geometry. Finally, remark that walls are present on each bank of the channel in figure 3. We introduced these walls to avoid dry areas.

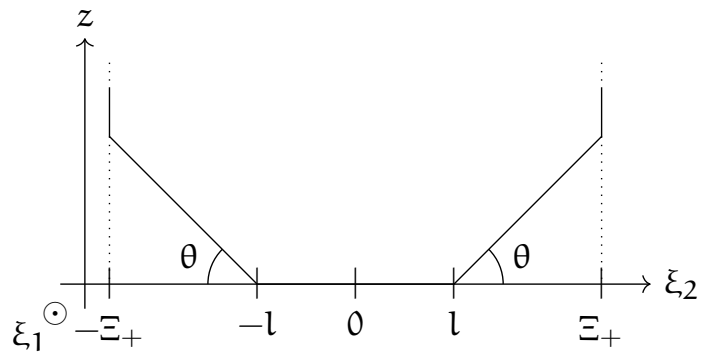

Figure 3: Sketch of the function $\phi\left(\xi_{2}\right)$.

For the sake of completeness, we finally provide the expression of the channel width $\mathrm{L}(z)$, as well as the correspondence between $\mathrm{S}$ and $\mathrm{H}$. According to (6.9), we have

$$
\mathrm{L}(z)= \begin{cases}2 l+\frac{2 z}{\tan \theta} & \text { if } 0 \leqslant z \leqslant\left(\Xi_{+}-l\right) \tan \theta, \\ 2 \Xi_{+} & \text {otherwise. }\end{cases}
$$

Therefore, since we assume $H>\left(\Xi_{+}-l\right) \tan \theta$ to avoid dry areas, we get

$$
S(H)=\int_{0}^{H} L(z) d z=2 H \Xi_{+}-\left(\Xi_{+}-l\right)^{2} \tan \theta .
$$

Chézy distribution. For the sake of simplicity, we consider a Chézy friction law. In dimensional form, the friction law reads $C\left(h, \xi_{1}, \xi_{2}\right)^{2}=C_{h}\left(\xi_{1}, \xi_{2}\right)^{2} h^{p}$. To determine the scaling parameter $\mathcal{C}_{0}$ of the whole friction law, we introduce the dimensionless Chézy friction coefficient, defined by $C_{h}:=\mathcal{C} c$. Therefore, we get $\mathcal{C}_{0}^{2}=\mathcal{C}^{2} \mathcal{H}^{p}$. For experiment \#1a, we take a uniform Chézy distribution, i.e. $c\left(\xi_{1}, \xi_{2}\right)=1$. For experiment \#1b, we set:

$$
c\left(\xi_{1}, \xi_{2}\right)=1+1.99 \frac{\xi_{2}-\left(\Xi_{-}+\Xi_{+}\right) / 2}{\Xi_{+}-\Xi_{-}} .
$$

For experiment \#2, the Chézy distribution is algorithmically computed according to the asymptotic regime and to the procedure detailed in section 6.1.2. Finally, for experiment \#3, we set

$$
c\left(\xi_{1}, \xi_{2}\right)=1.5-2 \frac{\left|\xi_{2}-\left(\Xi_{-}+\Xi_{+}\right) / 2\right|}{\Xi_{+}-\Xi_{-}} .
$$


Values of the reference parameters. We now give, for each of the three experiments performed in the remainder of this section, the chosen values of the reference parameters in tables 1 and 2, as well as the geometry and numerical discretization in table 3. The reference discharge $Q$ is defined by $\mathcal{Q}=\mathcal{H} U$. Experiment \#1, presented in section 6.2.2, concerns the simulation of backwater curves. In table 3, we highlight the two parts of this experiment, \#1a and \#1b, respectively corresponding to a U-shaped channel and to a trapezoidal channel. Experiment \#2, in section 6.2.3, provides a comparison with the 2D reference solution. Experiment \#3, in section 6.2.4, is the unsteady simulation of a flood. For this experiment, $X$ is computed according to the characteristic time of the flood. All numerical results will be presented in dimensionless variables.

\begin{tabular}{lcccccccc}
\hline parameter & $\mathrm{g}\left(\mathrm{m} \cdot \mathrm{s}^{-2}\right)$ & $\mathrm{p}$ & $\mathcal{X}(\mathrm{km})$ & $\mathrm{y}(\mathrm{m})$ & $\mathcal{Q}\left(\mathrm{m}^{3} \cdot \mathrm{s}^{-1}\right)$ & $\mathrm{I}_{0}$ & $\mathcal{C}\left(\mathrm{m}^{5 / 3} \cdot \mathrm{s}^{-1}\right)$ & $\mathrm{F}$ \\
\hline experiment \#1 & 9.81 & $4 / 3$ & 5 & 45 & 225 & $4 \times 10^{-4}$ & 25 & 0.075 \\
experiment \#2 & 9.81 & $4 / 3$ & 10 & 50 & 25 & $1 \times 10^{-3}$ & 20 & 0.1 \\
experiment \#3 & 9.81 & $4 / 3$ & $\sim 1.77$ & 90 & 40 & $1.6 \times 10^{-3}$ & 45 & 0.09 \\
\hline
\end{tabular}

Table 1: Values of the reference parameters for each experiment.

\begin{tabular}{lcccccc}
\hline parameter & $\mathcal{H}(\mathrm{m})$ & $\mathcal{U}\left({\left.\mathrm{m} . \mathrm{s}^{-1}\right)}^{-}\right.$ & $\mathrm{J}_{0}$ & $\delta$ & $\mathrm{R}_{\mathrm{l}}$ & $\varepsilon$ \\
\hline experiment \#1 & 7.68 & 0.651 & $4.47 \times 10^{-5}$ & $1.53 \times 10^{-3}$ & $9 \times 10^{-3}$ & 0.193 \\
experiment \#2 & 1.37 & 0.366 & $2.21 \times 10^{-4}$ & $1.37 \times 10^{-4}$ & $5 \times 10^{-3}$ & $6.18 \times 10^{-3}$ \\
experiment \#3 & 1.35 & 0.328 & $3.55 \times 10^{-5}$ & $7.65 \times 10^{-4}$ & $5.08 \times 10^{-2}$ & 0.175 \\
\hline
\end{tabular}

Table 2: Approximate values of the other parameters for each experiment, computed from Table 1.

\begin{tabular}{lcccccc}
\hline parameter & $\left(\xi_{1}\right)_{+}$ & $n_{\xi_{1}}$ & $l$ & $\theta$ & $\Xi_{+}$ & $n_{\xi_{2}}$ \\
\hline experiment \#1a & 5 & 500 & 0 & 0 & 0.5 & 100 \\
experiment \#1b & 5 & 500 & 0.25 & 45 & 0.5 & 100 \\
experiment \#2 & 1 & 200 & 0.15 & 60 & 0.5 & 20 \\
experiment \#3 & $\sim 36.7$ & 400 & 0.15 & 45 & 0.5 & 40 \\
\hline
\end{tabular}

Table 3: Values of the geometry and discretization parameters for each experiment.

\subsubsection{Numerical experiment: backwater curves}

We start with the simulation of backwater curves, presented in section 6.1.1, and labeled experiment \#1 in tables 1 to 3 . We stress that the backwater curves satisfy the mass conservation equation, which imposes a constant and uniform averaged discharge $Q$ for stationary solutions. We set the normal height $H_{n}$ equal to 1 , and we compute the uniform and constant discharge $Q$ according to this value of $H_{n}$ by taking $Q=Q^{(0)}$.

In the first part of this experiment, labeled \#1a, we consider a U-shaped channel with uniform friction, and we get $\mathrm{Q} \simeq 2.99$. Then, in experiment \#1b, we switch to a trapezoidal channel with non-uniform friction, to get $\mathrm{Q} \simeq 2.72$. The corresponding dimensional values are roughly equal to $650 \mathrm{~m}^{3} . \mathrm{s}^{-1}$, which characterizes a mild flood regime.

Let us make the important remark that usual hydraulic engineering models are able to recover the correct normal height $\mathrm{H}_{n}$, consistent with the full 2D systems. However, as soon as the channel is no longer U-shaped, these usual models are not zeroth-order accurate on the averaged discharge $\mathrm{Q}$. Therefore, compared to the usual models, even our zeroth-order accurate model is able to recover both the correct normal height $\mathrm{H}_{\mathrm{n}}$ and the correct zeroth-order constant and uniform discharge $\mathrm{Q}^{(0)}$. 
Let us consider the example of the trapezoidal channel described above. The process used by the usual models consists in forcing $\mathrm{H}_{n}=1$ by modifying $\mathrm{Q}^{(0)}$. The normal height for the $\mathrm{SW}$ model is given by (6.2). Therefore, to get $\mathrm{H}_{\mathrm{n}}=1$, the constant and uniform discharge would have to be $\mathrm{Q} \simeq 3.02$. This is far from being equal to $\mathrm{Q}^{(0)} \simeq 2.72$. As a consequence, for the usual models, either the normal height is correct and the constant discharge is incorrect (this is the usual approach), or the normal height is incorrect and the constant discharge is correct. In this set of numerical experiments, in order to highlight the shortcomings of the usual models, we elect to have the correct constant discharge. As a consequence, the normal height given by the SW model will be incorrect as soon as the channel is no longer U-shaped.

From section 6.1.1, we note that the backwater curves are governed by a system of ODEs, and thus we only require a single boundary condition on the height, $\mathrm{H}_{0}$, to drive this steady flow. For the experiments, we take $\mathrm{H}_{0}=\left(\mathrm{H}_{n}+9 \mathrm{H}_{\mathrm{c}}\right) / 10$, where $\mathrm{H}_{\mathrm{c}}$ is the critical height, defined by canceling the denominator of the $\mathrm{H}_{\xi_{1}}$ equation. For the A1 system, the enstrophy and potential are initialized by taking the values $\Psi^{(0)}$ and $\Pi^{(0)}$ corresponding to $\mathrm{H}_{0}$. The numerical results are presented in figure 4.
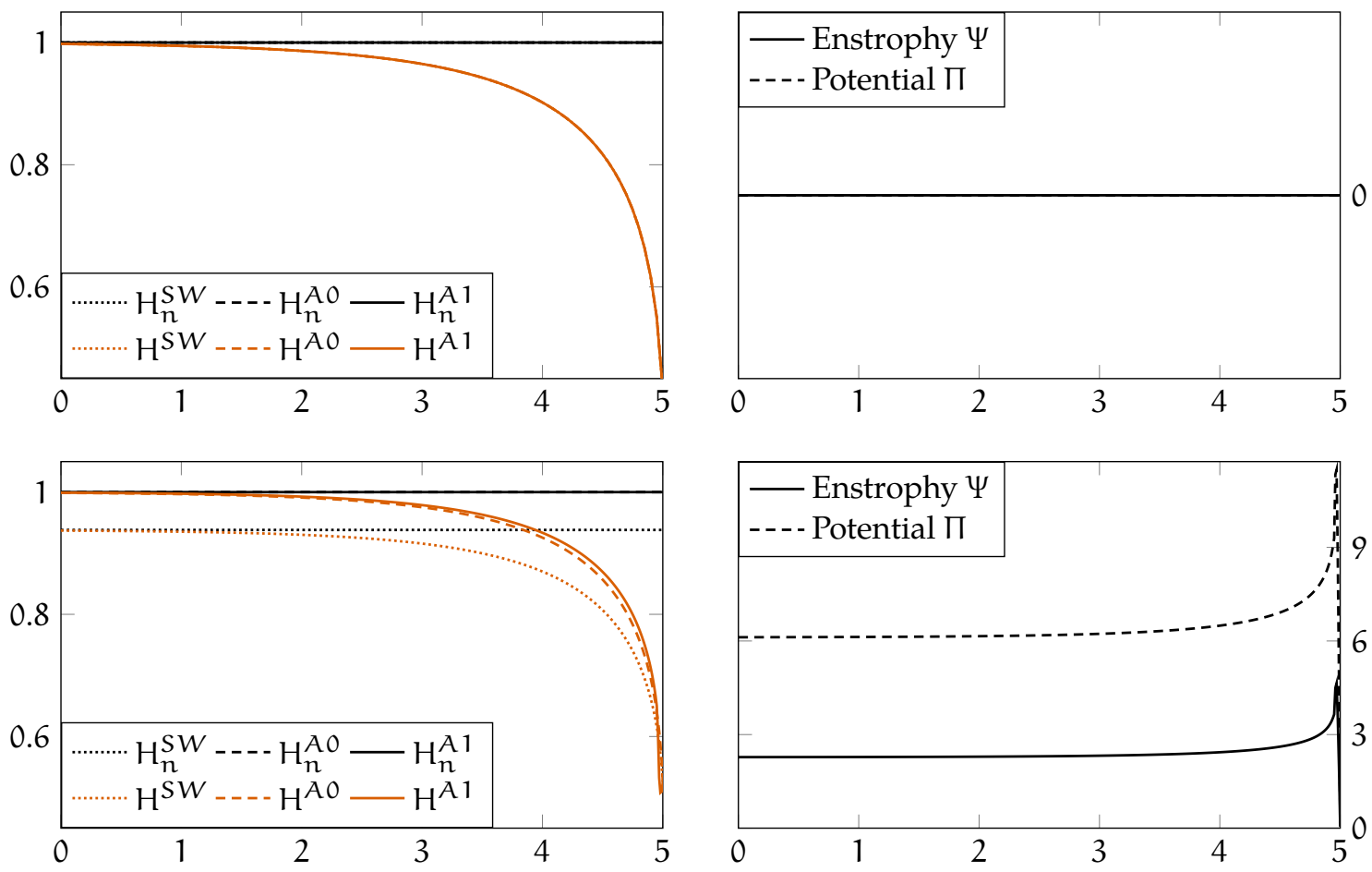

Figure 4: Simulation of backwater curves. Top panels: U-shaped channel with uniform friction. Bottom panels: trapezoidal channel with non-uniform friction. Left panel: height and normal height with respect to the position, for the three models. Right panel: enstrophy $\Psi$ and potential $\Pi$ for the A1 model.

In the left panels of figure 4 , we have represented the free surface $H$ for the three models, denoted in the legend by $H^{S W}, H^{A 0}$ and $H^{A 1}$, as well as the associated normal heights $H_{n}$ for each model. As expected, we note that the free surface $\mathrm{H}$ converges towards the relevant normal height when $\xi_{1}$ goes to 0 . Moreover, as explained in section 6.1.1, this normal height only depends on the friction model. Since we have the same friction model for the A0 and A1 models, the normal height associated to these models are the same, contrary to the one associated to the SW model, unless the channel is U-shaped. By comparing the two left panels of figure 4, we note that, as expected, the three models give the same results when the channel is U-shaped. However, as soon as it is no longer the case, the SW model greatly differs from the other two, which allows us to conclude that the SW 
model is irrelevant as soon as the channel is not U-shaped. We emphasize once again that the standard practice in hydraulic engineering is to force the correct normal height on the SW model. However, this approach does not recover the correct averaged discharge, and the resulting models are not zeroth-order accurate. Finally, note that the backwater curves of the A0 and A1 models are different when the channel is not U-shaped, up to $\mathcal{O}\left(\varepsilon^{2}\right)$, as expected.

In the right panels of figure 4 , we have displayed the enstrophy $\Psi$ and the potential $\Pi$ computed by the A1 model. As expected, these quantities vanish when the channel is U-shaped and the friction is uniform. In addition, once this is no longer the case, we note a similar behavior of these quantities compared to the height, in the sense that they tend towards a constant value when $\xi_{1}$ goes to 0 .

\subsubsection{Numerical experiment: comparison to $2 D$ steady solution}

We now turn to the comparison between the 2D reference steady solution from section 6.1.2 and the models under consideration.

Recall that the 2D solution is only driven by its boundary conditions $|\mathcal{F}| h\left(v_{1}\right)_{0}$ and $|\mathcal{F}| h\left(v_{2}\right)_{0}$ on the discharge, and that it will produce a steady flow at normal height $\mathrm{H}_{\mathrm{n}}^{2 \mathrm{D}}$. In order to compute this reference solution, we take $H_{\mathfrak{n}}^{2 D}=1,|\mathcal{F}| h\left(v_{1}\right)_{0} \simeq 0.75$ and $\| \mathcal{F}\left|h\left(v_{2}\right)_{0}\right|=\mathcal{O}(\varepsilon)$. These $2 \mathrm{D}$ results are presented in figure 5 .
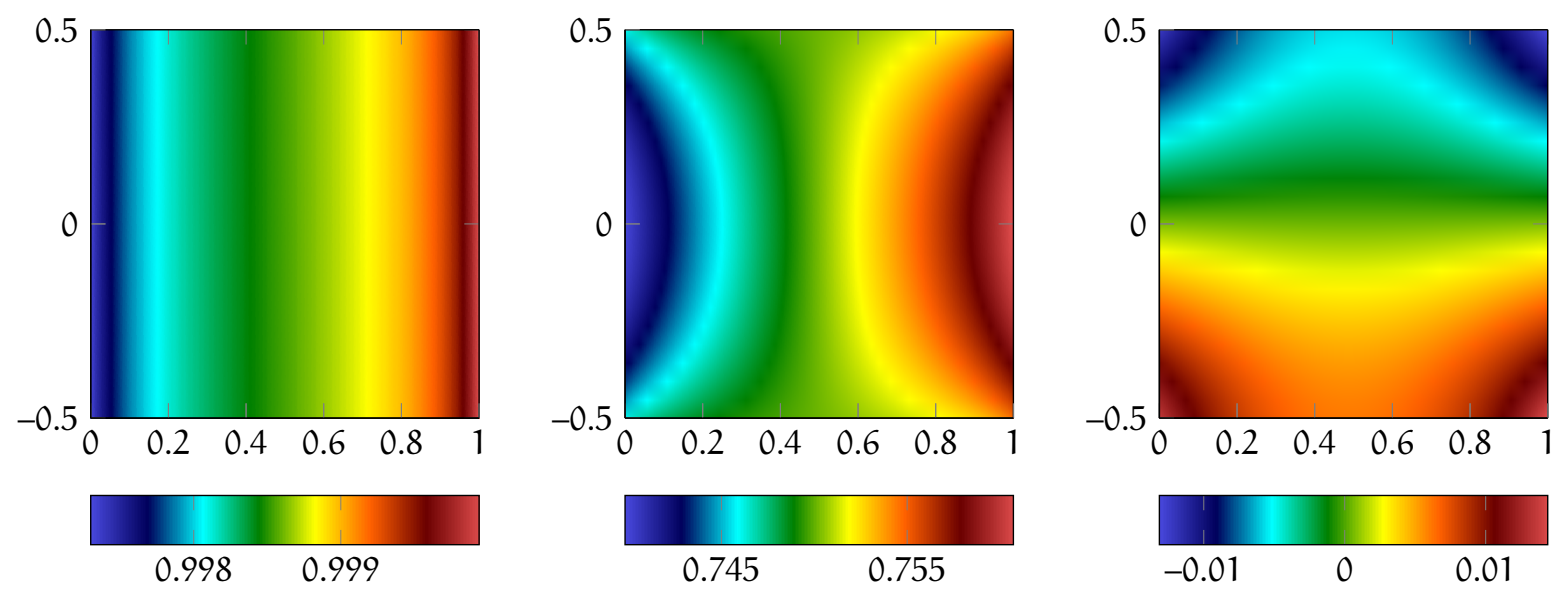

Figure 5: The 2D reference steady solution, computed from the process exhibited in section 6.1.2. From left to right: free surface $H\left(\xi_{1}\right)=$ $h\left(\xi_{1}, \xi_{2}\right)+\phi\left(\xi_{2}\right), \xi_{1}$-discharge $|\mathcal{F}| h \nu_{1}\left(\xi_{1}, \xi_{2}\right)$ and $\xi_{2}$-discharge $|\mathcal{F}| h \nu_{2}\left(\xi_{1}, \xi_{2}\right)$.

Equipped with this 2D solution, we can compare it to the three 1D models at our disposal. The constant and uniform 1D discharge is defined by $\mathrm{Q}=\int \Xi_{\Xi_{-}^{+}}^{\Xi_{+}}|\mathcal{F}| \mathrm{h} \nu_{1}(0.5, \mathrm{y}) \mathrm{d} \xi_{2}$. In figure 6 , we present the free surface $\mathrm{H}\left(\xi_{1}\right) \mathrm{com}-$ puted by the SW (dotted lines), A0 (dashed lines) and A1 (solid lines) models, in addition to the corresponding normal heights.

The left panel of figure 6 contains these comparisons. We note that the normal height associated to the SW model does not correspond at all to the $2 \mathrm{D}$ normal height (we get $\mathrm{H}_{\mathrm{n}}^{S W} \simeq 0.736$ while we have set $\mathrm{H}_{\mathrm{n}}^{2 \mathrm{D}}=1$ ). Thus, the free surface computed by the classical SW model is once again wildly inconsistent with the reference 2D solution. We once again stress that we have chosen to take the same averaged discharge for the three models, instead of fitting the correct $\mathrm{H}_{\mathrm{n}}^{\mathrm{SW}}$ as is usually the case in hydraulic engineering.

Turning to the right panel of figure 6, which displays a zoom of the left panel around the line $\mathrm{H}=1$, we observe that the A0 and A1 models are consistent with the 2D reference solution. Indeed, the small variations in free surface are due to the uniformity in space of the 1D discharge, compared to the 2D discharge whose divergence vanishes, but whose $\xi_{1}$-derivative is nonzero. Moreover, the $2 \mathrm{D}$ height becomes very close to the 1D 
ones around $\xi_{1}=0.5$, since the $1 \mathrm{D}$ discharge is the $\xi_{2}$-average of the $2 \mathrm{D}$ one at point $\xi_{1}=0.5$. In addition, we do not observe much difference between the solutions of the A0 and A1 models: the relative error in $\mathrm{L}^{2}$ norm is around $5 \times 10^{-5}$. This was to be expected since $\varepsilon \simeq 6.18 \times 10^{-3}$ in this experiment, and the difference between the $\mathrm{A} 0$ and $\mathrm{A} 1$ models is $\mathcal{O}\left(\varepsilon^{2}\right)$.
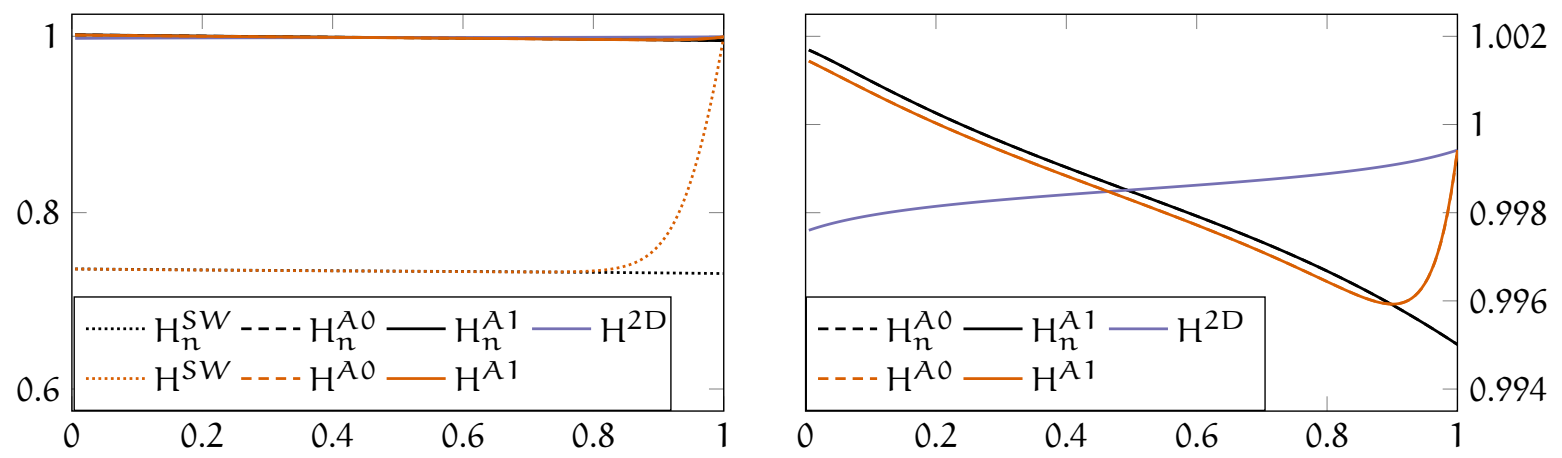

Figure 6: Comparison with the 2D steady reference solution. Left panel: height and normal height with respect to the position, for the three models; comparison with the 2D height. Right panel: zoom on the left panel to highlight the differences between the 2D height and the one computed by the $\mathrm{A} 0$ and $\mathrm{A} 1$ models.

Finally, in figure 7, we display the relative errors in $\mathrm{L}^{2}$ norm between the enstrophy $\Psi$ (left panel) or the potential $\Pi$ (right panel), computed by the A1 model, and their zeroth- and first-order approximations. As expected, these errors are $\mathcal{O}\left(\varepsilon^{2}\right)$, since the A1 model is consistent up to $\mathcal{O}(\varepsilon)$ in $\Psi$ and $\Pi$.
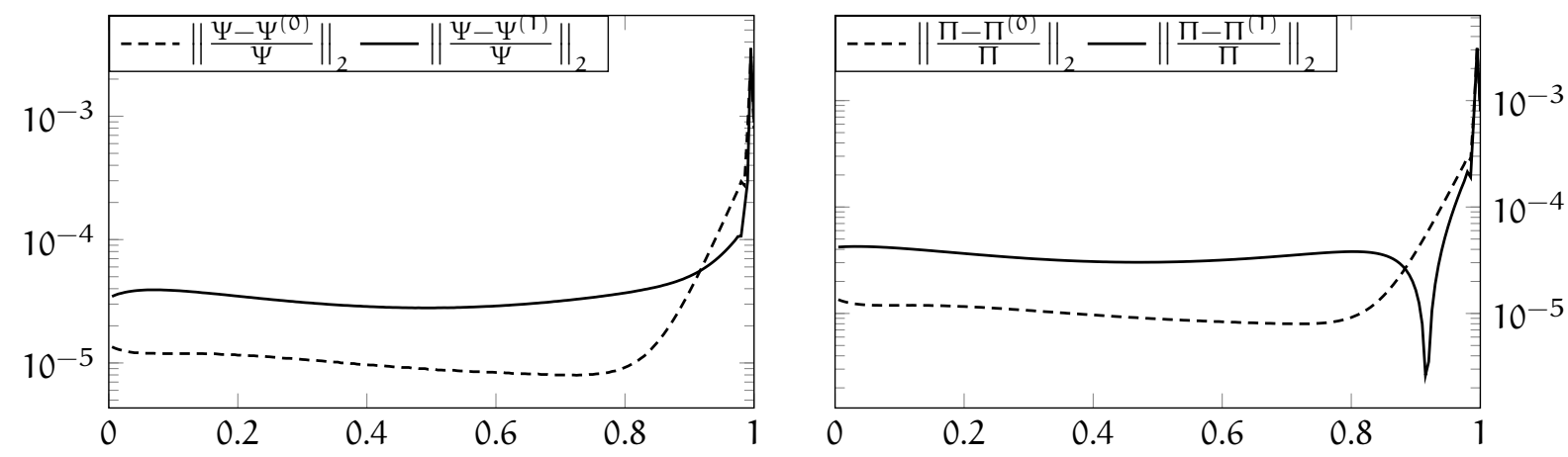

Figure 7: Errors between the computed enstrophy (left panel) or potential (right panel) and their zeroth- and first-order expansions, computed using the A1 model.

\subsubsection{Numerical experiment: unsteady flow}

In this final experiment, we consider the unsteady case of a flood. The reference solution is given by the DASSFLOW code $[8,24]$. Since the SW model has been proven to be inconsistent by the previous two experiments, we no longer consider it. We compare the A0 and A1 models, discretized as explained in section 6.1.3, to the reference solution.

In addition, we consider the kinematic waves $(\mathrm{KW})$ approximation, which consists in taking the mass conservation equation of the shallow water equations and considering $Q=Q^{(0)}(S)$, as follows:

$$
S_{t}+\left(Q^{(0)}(S)\right)_{\xi_{1}}=0
$$

Since $\mathrm{Q}^{(0)}$ depends non-linearly on $\Lambda$, it depends on $S_{\xi_{1}}$. The kinematic wave equation therefore is a nonlinear 
diffusion equation, which we discretize with a classical upwind scheme. Note that this numerical scheme involves a restrictive stability condition on the time step, in $\Delta t=\mathcal{O}\left(\Delta x^{2}\right)$.

The reference parameters for this experiment correspond to the Garonne river upstream of Toulouse (see tables 1 and 3). We consider a flood lasting a dimensional time of 7.5h, and the total dimensional time of the unsteady experiment is $10 \mathrm{~h}$. The discharge increases during the dimensional time $\mathrm{T}=1.5 \mathrm{~h}$, then stagnates during the dimensional time $1.5 \mathrm{~h}$, and finally decreases to the initial level during the dimensional time $4.5 \mathrm{~h}$. We get the characteristic length $X$ from the characteristic flood time $T$ by setting $X=U T \simeq 1.772 \mathrm{~km}$. The total dimensional length is $65 \mathrm{~km}\left(\xi_{1} \in[0,65 / X]\right.$, where $\left.65 / X \simeq 36.69\right)$, and we consider a probe at dimensional position $62.25 \mathrm{~km}\left(\xi_{1} \simeq 34.57\right)$. This domain roughly corresponds to the Marquefave-Toulouse portion of the Garonne river.

The free surface is initialized at the normal height. From this normal height, we compute the initial discharge $Q_{n}$, enstrophy and potential through their respective zeroth-order asymptotic expansions. As a consequence, the flow of water in the river is a steady solution before the flood begins. The flood itself is simulated by a time-dependent left boundary condition on the discharge $Q$. We take $Q(t, 0)=Q_{\text {in }}(t)$, with

$$
Q_{i n}(t)= \begin{cases}Q_{n}+\left(\frac{2200}{Q}-Q_{n}\right) \frac{t}{0.15 t_{\text {end }}} & \text { if } t \leqslant 0.15 t_{\text {end }}, \\ \frac{2200}{Q} & \text { if } 0.15 t_{\text {end }} \leqslant t \leqslant 0.30 t_{\text {end }} \\ Q_{n}+\left(\frac{2200}{Q}-Q_{n}\right) \frac{0.75 t_{\text {end }}-t}{0.75 t_{\text {end }}-0.30 t_{\text {end }}} & \text { if } 0.30 t_{\text {end }} \leqslant t \leqslant 0.75 t_{\text {end }} \\ Q_{n} & \text { otherwise, }\end{cases}
$$

where $t_{\text {end }}$ is the dimensionless final time. This boundary condition ensures a dimensional peak flood discharge of $2200 \mathrm{~m}^{3} \cdot \mathrm{s}^{-1}$, which corresponds to a 5-year flood for the Garonne river. The left boundary condition on the section is obtained by computing $S_{i n}(t)$ such that $Q_{i n}(t)=Q^{(0)}\left(S_{i n}(t)\right)$. The left boundary conditions on the enstrophy and potential are their respective zeroth-order asymptotic expansions. Finally, we consider homogeneous Neumann boundary conditions on the right boundary. Regarding the 2D code, the top and bottom boundaries are solid walls, and we prescribe standard 2D inflow and outflow boundary conditions at the left and right boundaries, respectively. These boundary conditions distribute the $1 \mathrm{D}$ discharge $\mathrm{Q}_{n}$ over the whole channel, taking into account the specific geometry of the flow. From this discharge, the water height is computed at the boundaries.

In figure 8, we display the free surface $\mathrm{H}$ (left panels) and discharge $\mathrm{Q}$ (right panels) at dimensional times $2 \mathrm{~h}$ (top panels) and $9 \mathrm{~h}$ (bottom panels). The solid blue line with $\times$ marks represents the width-averaged 2D reference solution. The dashed line and the solid line with + marks respectively represent the results of the A0 and A1 models. The results of the kinematic waves approximation, labeled $\mathrm{H}^{\mathrm{KW}}$ and $\mathrm{Q}^{\mathrm{KW}}=\mathrm{Q}^{(0)}\left(\mathrm{H}^{\mathrm{KW}}\right)$, are represented with dotted lines. On each panel, we magnify the point where the differences between the four models are the largest. The magnification level for each panel is written under the line connecting the magnifying glass to the graphs.

We consistently observe that the A1 model yields the best approximation of the 2D reference solution, as expected since it recovers the first-order expansion of the discharge. The approximation obtained with the KW model is worse than the one coming from the A0 model, even if both recover the zeroth-order expansion $\mathrm{Q}^{(0)}$ of the discharge. The most striking differences between the models are located where the flood advances on the steady river. This denotes a difference between the zeroth-order and first-order friction models.

To get a better understanding of the relevance of the A1 model compared to the A0 one, we now display 

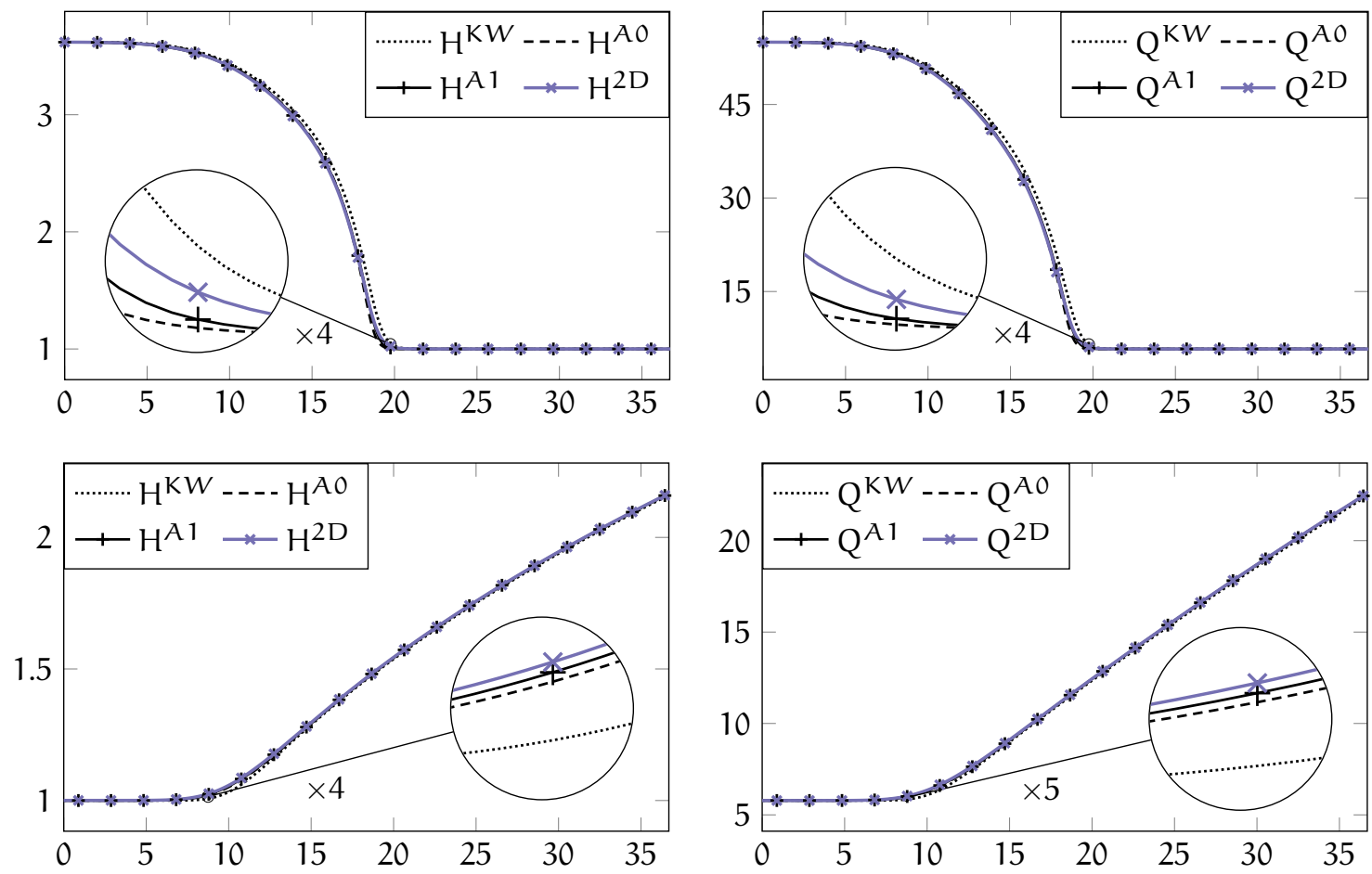

Figure 8: Free surface $\mathrm{H}$ (left panels) and discharge $\mathrm{Q}$ (right panels) for the flood experiment. Comparison of the 2D reference solution with the A0 and A1 models, as well as the kinematic waves (KW) approximation. The results are displayed with respect to the position, at dimensional times $t=2 \mathrm{~h}$ (top panels) and $t=9 \mathrm{~h}$ (bottom panels). Magnification of the zones where the three models yield the most different results, with magnification level written below the line linking the magnifying glass to the graphs.

in figure 9 the relative errors in space between the 2D reference solution and the models with respect to the dimensional time. The relative spatial errors in $\mathrm{L}^{2}$ norm are computed as follows:

$$
\left\|\frac{\mathrm{H}^{\mathrm{A} 1}(\cdot, \mathrm{t})-\mathrm{H}^{2 \mathrm{D}}(\cdot, \mathrm{t})}{\mathrm{H}^{2 \mathrm{D}}(\cdot, \mathrm{t})}\right\|_{\mathrm{L}^{2}}=\sqrt{\int_{\xi_{1}}\left|\frac{\mathrm{H}^{\mathrm{A} 1}\left(\xi_{1}, \mathrm{t}\right)-\mathrm{H}^{2 \mathrm{D}}\left(\xi_{1}, \mathrm{t}\right)}{\mathrm{H}^{2 \mathrm{D}}\left(\xi_{1}, \mathrm{t}\right)}\right|^{2} \mathrm{~d} \xi_{1},}
$$

and the relative spatial errors in $\mathrm{L}^{\infty}$ norm as defined by:

$$
\left\|\frac{\mathrm{H}^{\mathrm{A} 1}(\cdot, \mathrm{t})-\mathrm{H}^{2 \mathrm{D}}(\cdot, \mathrm{t})}{\mathrm{H}^{2 \mathrm{D}}(\cdot, \mathrm{t})}\right\|_{\mathrm{L}^{\infty}}=\max _{\xi_{1}}\left|\frac{\mathrm{H}^{\mathrm{A} 1}\left(\xi_{1}, \mathrm{t}\right)-\mathrm{H}^{2 \mathrm{D}}\left(\xi_{1}, \mathrm{t}\right)}{\mathrm{H}^{2 \mathrm{D}}\left(\xi_{1}, \mathrm{t}\right)}\right| .
$$

In the left panel of figure 9, we display the errors in $\mathrm{L}^{2}$ norm, and we display the errors in $\mathrm{L}^{\infty}$ norm in its right panel. The errors made by the KW, A0 and A1 models are respectively depicted with dotted lines, dashed lines and solid lines.

We observe, like in figure 8, that the KW model produces a worse approximation than the A0 model, whose approximation is itself worse than the one given by A1 model. Indeed, the maximum over time of the spatial error in $\mathrm{L}^{2}$ norm is around $23.6 \%$ for the $\mathrm{KW}$ model, $11.3 \%$ for the $\mathrm{A} 0$ model and $3.17 \%$ for the $\mathrm{A} 1$ model. The maximum over time of the spatial error in $\mathrm{L}^{\infty}$ norm is around $22.6 \%$ for the KW model, $14.0 \%$ for the A0 model and $3.81 \%$ for the A1 model. Once again, the advantages of the A1 model over the other two models are undeniable. Note that similar conclusions can be obtained from the study of the discharge errors, and we do not display the error curves here for the sake of conciseness.

In the left panels of figure 10, we display the relative errors between the reference solution and the models, with respect to time and at the probe located at $\xi_{1} \simeq 34.57$. The top left panel displays the error on $\mathrm{H}$ while 

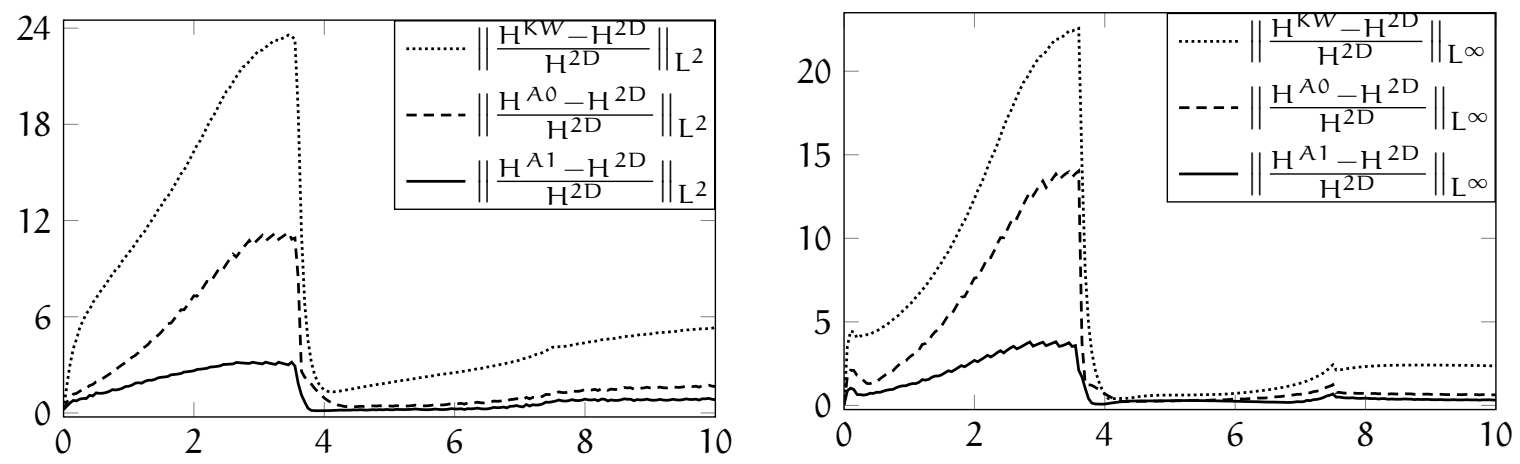

Figure 9: Relative errors in space in $\mathrm{L}^{2}$ norm (left panel) and in $\mathrm{L}^{\infty}$ norm (right panel) between the 2D reference solution and the KW model (dotted lines), the A0 model (dashed lines) and the A1 model (solid lines), represented with respect to the dimensional time.

the bottom left panel displays the error on $\mathrm{Q}$. The errors between the 2D reference solution and the A0 and A1 models are respectively represented with a dashed line and a solid line. In the right panels, we display a zoom around the point $\xi_{1} \simeq 34.57$ at dimensional time $3.35 \mathrm{~h}$, when the errors are the largest. The top right panel displays $\mathrm{H}$ and the bottom right one displays $\mathrm{Q}$. The color coding is the same as in figure 8.

The observations we had made in figure 8 are confirmed by figure 10. Indeed, at the probe, the largest difference in free surface $\mathrm{H}$ are around $11.9 \%$ for the $\mathrm{A} 0$ model and $2.00 \%$ for the $\mathrm{A} 1$ model. Regarding the discharge $\mathrm{Q}$, the largest differences at the probe are about $25.9 \%$ for the A0 model and $4.86 \%$ for the A1 model. These differences are obtained around dimensional time $3.35 \mathrm{~h}$, when the peak of the advancing flood reaches the probe at $\xi_{1} \simeq 34.57$. Note that the differences between the reference solution and the zeroth-order models are closely related to the values of $\varepsilon \simeq 0.175$ and $\varepsilon^{2} \simeq 3.06 \times 10^{-2}$.

\section{Conclusion and summary of the results}

We have presented a novel approach to derive 1D models for river flows that are consistent with the usual 2D shallow water equations (1.1). These models are based on asymptotic expansions with respect to a new parameter $\varepsilon$, defined in (3.1). We conclude this paper with a concise summary of the new models we have developed, as well as with some words on their range of applicability, and a summary of the numerical experiments presented in section 6 .

\subsection{Summary of the two models}

Considering the small parameter

$$
\varepsilon=\frac{\mathcal{C}_{0}^{2}}{g X^{\prime}}
$$

which is a geometrical term linking the friction scaling to the typical wavelength, we have obtained asymptotic expansions with respect to $\varepsilon$ of the solutions of the 2D shallow water system (2.4). Then, we have developed two one-dimensional models, whose solutions deviate in $\mathcal{O}(\varepsilon)$ or $\mathcal{O}\left(\varepsilon^{2}\right)$ from the asymptotic expansions. In this section, we first recall the zeroth-order model (with an $\mathcal{O}(\varepsilon)$ deviation) and we then summarize the first-order model (with an $\mathcal{O}\left(\varepsilon^{2}\right)$ deviation). A few words are finally given on the range of applicability of these models.

\subsubsection{Zeroth-order model}

We consider an arbitrarily meandering river. It can be strongly meandering, i.e. $R_{y}=\mathcal{O}(1)$, or weakly meandering, i.e. $R_{y}=\mathcal{O}(\varepsilon)$. The zeroth-order model, hyperbolic and linearly stable, is then given by (4.6), or, 

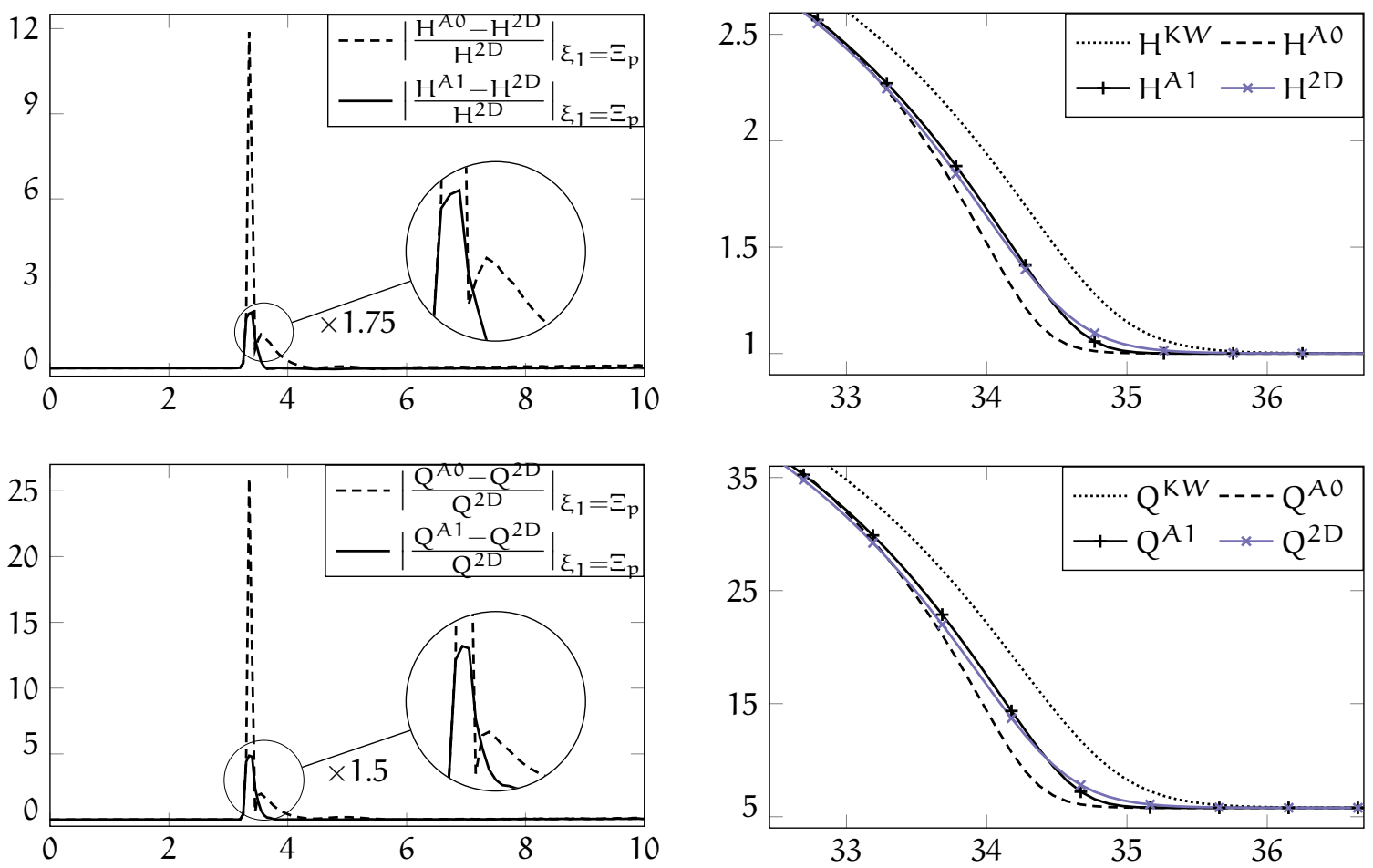

Figure 10: Left panels: relative errors between the 2D reference solution and the A0 and A1 models (the dashed line for the A0 model and the solid line for the A1 model). The errors on $\mathrm{H}$ are displayed in the top left panel and the ones on Q are depicted in the bottom left panel. These errors are computed at dimensionless position $\xi_{1} \simeq 34.57$ and are plotted with respect to the dimensional time. Right panels: free surface $\mathrm{H}$ (on top) and discharge Q (at the bottom) for the flood experiment, computed with the 2D system as well as the A0 and A1 models, and displayed with respect to the position, at dimensional time 3.35h. Magnification of the zones where the three models yield the most different results, with magnification level written below the line linking the magnifying glass to the graphs.

equivalently, by:

$$
\left\{\begin{array}{l}
\mathrm{S}_{\mathrm{t}}+\mathrm{Q}_{\xi_{1}}=0 \\
\mathrm{Q}_{\mathrm{t}}+\left(\frac{\mathrm{Q}^{2}}{\mathrm{~S}}\right)_{\xi_{1}}=\frac{1}{\varepsilon} \mathrm{S}\left(\Lambda-J_{\mathrm{m}}\right) .
\end{array}\right.
$$

In (7.1), $S$ is the wetted section, $H$ the free surface, and $Q$ the section-averaged discharge. Recall that $S$ can be obtained from $\mathrm{H}$, and reciprocally, using the formula (3.4).In addition, $J$ is the main longitudinal slope and $\Lambda$ is a corrected slope, defined by

$$
\Lambda=\mathcal{J}-\frac{\delta}{\mathrm{J}_{0}} \mathrm{H}_{\xi_{1}}
$$

The zeroth-order asymptotic expansion used in (7.1) is defined by

$$
\mathrm{Q}^{(0)}=\sqrt{|\Lambda|} \operatorname{sgn}(\Lambda) \int_{\Xi_{-}}^{\Xi_{+}}(\mathrm{H}-\phi) \frac{\mathrm{C}}{\sqrt{|\mathcal{F}|}} \mathrm{d} \xi_{2}, \quad \text { where }|\mathcal{F}|=1-\mathrm{R}_{\mathrm{y}} \xi_{2} \frac{\sigma\left(\xi_{1}\right)}{\mathrm{R}\left(\xi_{1}\right)}
$$

The friction source term $\mathcal{J}_{m}$ is defined by

$$
\partial_{m}=\Lambda \frac{Q|Q|}{Q^{(0)}\left|Q^{(0)}\right|}=\frac{Q|Q|}{\left(C_{h}^{2}\right)_{m} R_{h} S^{2}}, \quad \text { where }\left(C_{h}^{2}\right)_{m}=\frac{1}{R_{h} S^{2}}\left(\int_{\Xi_{-}}^{\Xi_{+}}(H-\phi) \frac{C}{\sqrt{|\mathcal{F}|}} d \xi_{2}\right)^{2} .
$$

The discharge equation from (7.1) deviates with $\mathcal{O}(1)$ from the integrated longitudinal velocity equation $(2.9 \mathrm{~b})$. As a consequence, a Chapman-Enskog asymptotic expansion of the discharge in (7.1) yields $\mathrm{Q}=\mathrm{Q}^{(0)}+\mathcal{O}(\varepsilon)$, and the model is zeroth-order accurate. 
Furthermore, note that the zeroth-order model (7.1) has the same form that the usual hydraulic engineering models (see for instance $[6,36]$ ). The only difference comes from the friction model $(7.3)$, which seems to be a new feature of this model.

\subsubsection{First-order model}

We now consider only a weakly meandering river, i.e. $R_{y}=\mathcal{O}(\varepsilon)$. The first-order model, hyperbolic and linearly stable, is then given by (5.24), or, equivalently, by:

$$
\left\{\begin{array}{l}
S_{\mathrm{t}}+\mathrm{Q}_{\xi_{1}}=0, \\
\mathrm{Q}_{\mathrm{t}}+\left(\frac{\mathrm{Q}^{2}}{\mathrm{~S}}+\mathrm{S} \Psi\right)_{\xi_{1}}=\frac{1}{\varepsilon} \mathrm{S}\left(\Lambda-\mathcal{J}-\frac{\mathrm{S}^{2} \Psi^{(0)}}{\left(\mathrm{Q}^{(0)}\right)^{2}}\left(\Lambda-\partial_{\Psi}\right)\right) \\
\left(\frac{1}{2} \frac{\mathrm{Q}^{2}}{\mathrm{~S}}+\frac{1}{2} S \Psi\right)_{\mathrm{t}}+\left(\frac{\mathrm{Q}}{\mathrm{S}}\left(\frac{1}{2} \frac{\mathrm{Q}^{2}}{\mathrm{~S}}+\frac{1}{2} S \Pi\right)\right)_{\xi_{1}}=\frac{1}{\varepsilon} \mathrm{Q}(\Lambda-J), \\
\left(\frac{1}{2} S(\Pi-3 \Psi)\right)_{\mathrm{t}}=\frac{1}{\varepsilon} \mathrm{Q} \frac{S^{2} \Pi^{(0)}}{\left(\mathrm{Q}^{(0)}\right)^{2}}\left(\partial_{\Psi}-\partial_{\Pi}\right) .
\end{array}\right.
$$

In (7.4), $\mathrm{S}$ is the wetted section, $\mathrm{H}$ the free surface, $\mathrm{Q}$ the section-averaged discharge, $\Psi$ and $\Pi$ the enstrophy and the potential, defined by:

$$
S \Psi=\int_{\Xi_{-}}^{\Xi_{+}}|\mathcal{F}| \mathrm{h}\left(v_{1}-\mathrm{U}\right)^{2} \mathrm{~d} \xi_{2} \geqslant 0 \quad \text { and } \quad S \Pi=\int_{\Xi_{-}}^{\Xi_{+}}|\mathcal{F}| \mathrm{h}\left(v_{1}-\mathrm{U}\right)^{2}\left(2+\frac{v_{1}}{\mathrm{u}}\right) \mathrm{d} \xi_{2} \geqslant 0 .
$$

In addition, $\mathcal{J}$ is the main longitudinal slope and $\Lambda$ is a corrected slope, defined by (7.2). The zeroth-order asymptotic expansions used in (7.4) are defined by

$$
\mathrm{Q}^{(0)}=\sqrt{|\Lambda|} \operatorname{sgn}(\Lambda) \mathcal{M}_{1}, \quad S \Psi^{(0)}=|\Lambda|\left(\mathcal{M}_{2}-\frac{\mathcal{M}_{1}^{2}}{\mathcal{M}_{0}}\right), \quad S \Pi^{(0)}=|\Lambda|\left(\frac{\mathcal{M}_{0} \mathcal{M}_{3}}{\mathcal{M}_{1}}-\frac{\mathcal{M}_{1}^{2}}{\mathcal{M}_{0}}\right),
$$

where we have introduced the shorter notation

$$
\mathcal{M}_{n}=\int_{\Xi_{-}}^{\Xi_{+}}(H-\phi)|\mathcal{F}|\left(\frac{C}{|\mathcal{F}|^{3 / 2}}\right)^{n} d \xi_{2} .
$$

The friction source term $\mathcal{J}$ is taken equal to $\mathcal{J}_{m}$, defined by (7.3). The other two relaxation source terms are defined by

$$
\partial_{\Psi}=\operatorname{sgn}(Q)|\Lambda| \frac{\Psi}{\Psi^{(0)}} \quad \text { and } \quad \partial_{\Pi}=\operatorname{sgn}(Q)|\Lambda| \frac{\Pi}{\Pi^{(0)}} .
$$

The discharge equation from (7.1) deviates with $\mathcal{O}(1)$ from the integrated longitudinal velocity equation $(2.9 \mathrm{~b})$. Similarly, a $\mathcal{O}(1)$ error is introduced in the fourth equation of (7.1). However, the energy equation from (7.1) deviates with $\mathcal{O}(\varepsilon)$ from the integrated energy equation (2.9c). As a consequence, performing a Chapman-Enskog asymptotic expansion of the discharge in (7.1) yields a $\mathrm{Q}=\mathrm{Q}^{(0)}+\varepsilon \mathrm{Q}^{(1)}+\mathcal{O}\left(\varepsilon^{2}\right)$, and the model is first-order accurate.

In addition, note that the first-order structure naturally involves the enstrophy $\Psi$, which gives a better understanding of the cross-stream velocity variations. Such a quantity was also introduced in $[17,28]$ to enhance the $2 \mathrm{D}$ shallow water equations with vertical velocity variations. Therefore, the enstrophy seems to be a natural quantity to consider when modeling complex water flow phenomena. 
Finally, remark that the energy equations of the zeroth- and first-order models can be rewritten to highlight that the energy is only dissipated due to the friction. Let us note that the following identity holds:

$$
\frac{1}{\varepsilon} \mathrm{Q} \wedge=-\left(\varepsilon+\frac{1}{\varepsilon} \frac{\mathrm{I}_{0}}{\mathrm{~J}_{0}} \mathrm{Sb}_{0}\right)_{\mathrm{t}}-\left(\frac{\mathrm{Q}}{\varepsilon}\left(\frac{\mathrm{I}_{0}}{\mathrm{~J}_{0}} \mathrm{~b}_{0}+\frac{\delta}{\mathrm{J}_{0}} \mathrm{H}\right)\right)_{\xi_{1}}{ }^{\prime}
$$

where $\mathcal{E}=\frac{1}{\mathrm{~F}^{2}} \int_{0}^{\mathrm{H}} z \mathrm{Ld} z$ is the potential gravity energy. Using (7.5), the energy equation of the zeroth-order model (7.1) reads as follows:

$$
\left(\frac{1}{2} \frac{\mathrm{Q}^{2}}{\mathrm{~S}}+\mathcal{E}+\frac{1}{\varepsilon} \frac{\mathrm{I}_{0}}{\mathrm{~J}_{0}} \mathrm{~S} \mathrm{~b}_{0}\right)_{\mathrm{t}}+\left(\frac{\mathrm{Q}}{\mathrm{S}}\left[\frac{1}{2} \frac{\mathrm{Q}^{2}}{\mathrm{~S}}+\frac{\mathrm{S}}{\varepsilon}\left(\frac{\mathrm{I}_{0}}{\mathrm{~J}_{0}} \mathrm{~b}_{0}+\frac{\delta}{\mathrm{J}_{0}} \mathrm{H}\right)\right]\right)_{\xi_{1}}=-\frac{\mathrm{Q}_{1}}{\varepsilon} \partial
$$

while the one of the first-order model (7.4) can be rewritten as:

$$
\left(\frac{1}{2} \frac{Q^{2}}{S}+\frac{1}{2} S \Psi+\varepsilon+\frac{1}{\varepsilon} \frac{I_{0}}{J_{0}} S b_{0}\right)_{t}+\left(\frac{Q}{S}\left[\frac{1}{2} \frac{Q^{2}}{S}+\frac{1}{2} S \Pi+\frac{S}{\varepsilon}\left(\frac{I_{0}}{J_{0}} b_{0}+\frac{\delta}{J_{0}} H\right)\right]\right)_{\xi_{1}}=-\frac{Q^{2}}{\varepsilon} \partial .
$$

These formulations also emphasize the main difference between the zeroth- and first-order models. Namely, introducing the terms in $\Psi$ and $\Pi$ in the first-order model is enough to ensure a $\mathcal{O}(\varepsilon)$ deviation from the asymptotic expansions, compared to the $\mathcal{O}(1)$ deviation present in the zeroth-order model. In addition, these formulations are nothing but the $1 \mathrm{D}$ versions of the $2 \mathrm{D}$ energy equation (2.8).

\subsubsection{Range of applicability of the models}

We finish with a few words on the range of applicability of the two models. The two models have been derived with an error up to $\mathcal{O}(\varepsilon)$ for the zeroth-order model and $\mathcal{O}\left(\varepsilon^{2}\right)$ for the first-order models. Therefore, they are applicable as soon as the parameter $\varepsilon$ is not too large. This condition is indeed satisfied in a typical river, see the discussion at the beginning of section 3.

However, one may wish to consider large Froude numbers. To better understand this case, we note that (3.12) prescribes the free surface as follows:

$$
h+\phi=H-F^{2} R_{y} \frac{\sigma}{R}|\Lambda| \int_{0}^{\xi_{2}} \frac{C(H-\phi)^{2}}{|\mathcal{F}|^{2}} \mathrm{~d} \eta+\mathcal{O}\left(F^{4}\right) .
$$

We immediately observe that, if both $F^{2}=\mathcal{O}(1)$ and $R_{y}=\mathcal{O}(1)$, i.e. if we are in a strongly meandering situation with a large Froude number, the free surface is no longer flat up to $\mathcal{O}(\varepsilon)$. This will make it harder to compute $\mathrm{H}$ from $\mathrm{S}$ and $\mathrm{S}$ from $\mathrm{H}$.

This means that the 1D models are harder to apply in a strongly meandering, high Froude number case, since the relationship between $S$ and $\mathrm{H}$ becomes more complex. Outside of this specific case, the model is applicable without restriction on the Froude number, as long as $\varepsilon$ is not too large.

\subsection{Summary of the numerical results}

In this paper, we have presented three numerical experiments: the computation of backwater curves in section 6.2.2, the comparison to a 2D steady solution in section 6.2.3, and the simulation of a flood flow in an idealized river similar to the Garonne in section 6.2.4.

The backwater curves experiment showed that, as soon as the river is no longer U-shaped or the friction is no longer uniform in the transverse direction, the usual 1D hydraulic engineering models fail to capture the correct discharge for a steady flow, while our two models manage to capture both the correct normal height and the correct discharge. 
This result is confirmed when comparing the usual and new models to a $2 \mathrm{D}$ reference steady solution. In this case, only the new models allow to capture both the correct normal height and discharge, which further emphasizes the need to use consistent 1D models when considering non-uniform geometry or friction distributions.

Finally, the Garonne-like experiment shows that, even though the zeroth-order model is consistent with the 2D system, it can still produce an error of up to $15 \%$ on the free surface and $25 \%$ on the discharge, compared to the 2D equations. Using the first-order model reduces these errors to around $3.8 \%$ and $4.9 \%$, This experiment highlights the relevance of first-order models over zeroth-order ones when considering an unsteady flow.

Acknowledgments. The authors extend their thanks to the Service d'Hydrographie et d'Océanographie de la Marine (SHOM) for financial support. The authors would like to warmly thank Frédéric Couderc for his help in setting up the DASSFLOW flood experiment as well as Jérôme Monnier for his valuable comments which greatly improved the clarity of the results. The authors also thank the anonymous reviewers for their insightful comments and detailed remarks.

\section{Appendix A. Writing the 2D shallow water system in local coordinates}

With the definitions from section 2.1, we have the following chain rules:

Lemma A.1. For any vector fields $\boldsymbol{z}$ and $\boldsymbol{v}=A \boldsymbol{u}$, the differential operators transform according to

$$
|\mathfrak{F}| \nabla_{\mathbf{X}} \cdot \boldsymbol{z}=\nabla_{\boldsymbol{\xi}} \cdot(|\mathcal{F}| A z), \quad \boldsymbol{u} \cdot \nabla_{\mathbf{X}}=\boldsymbol{v} \cdot \nabla_{\xi}, \quad \nabla_{\mathbf{X}}=A^{\top} \nabla_{\xi}
$$

Let us rewrite (1.1) in this new system of coordinates. We first consider the mass conservation law. According to lemma A.1, we get:

$$
|\mathcal{F}| h_{\mathbf{t}}=-|\mathcal{F}| \nabla_{\mathbf{X}} \cdot(\mathbf{h} \mathbf{u})=-\nabla_{\xi} \cdot(|\mathcal{F}| \mathrm{h} A \mathbf{u})=-\nabla_{\xi} \cdot(|\mathcal{F}| \mathrm{h} \boldsymbol{v}) .
$$

The mass conservation law in this new system of coordinates reads

$$
|\mathcal{F}| h_{t}+\nabla_{\xi} \cdot(|\mathcal{F}| h \boldsymbol{v})=0
$$

Let us now consider the discharge balance law. Using lemma A.1, we get

$$
\boldsymbol{v}_{\mathrm{t}}+A\left(\boldsymbol{v} \cdot \nabla_{\boldsymbol{\xi}}\left(A^{-1} \boldsymbol{v}\right)\right)+g A A^{\top} \nabla_{\boldsymbol{\xi}}(\mathrm{h}+\mathrm{Z})=-\frac{g\left\|A^{-1} \boldsymbol{v}\right\|}{\mathrm{C}(\mathrm{h}, \mathrm{a}(\boldsymbol{\xi}))^{2}} \boldsymbol{v} .
$$

To simplify notations, we introduce in this Appendix $c_{\theta}=\cos \left(\theta\left(\xi_{1}\right)\right)$ and $s_{\theta}=\cos \left(\theta\left(\xi_{1}\right)\right)$, to rewrite $A^{-1}$ and $A$ as

$$
A^{-1}=\left(\begin{array}{cc}
c_{\theta}|\mathcal{F}| & -s_{\theta} \\
s_{\theta}|\mathcal{F}| & c_{\theta}
\end{array}\right) \quad \text { and } \quad A=\frac{1}{|\mathcal{F}|}\left(\begin{array}{cc}
c_{\theta} & s_{\theta} \\
-s_{\theta}|\mathcal{F}| & c_{\theta}|\mathcal{F}|
\end{array}\right) .
$$

We need to compute $A A^{\top}$ and $A\left(\boldsymbol{v} \cdot \nabla_{\xi}\left(A^{-1} \boldsymbol{v}\right)\right)$. We immediately get $A A^{\top}=\operatorname{diag}\left(|\mathcal{F}|^{-2}, 1\right)$; let us now compute $A\left(\boldsymbol{v} \cdot \nabla_{\xi}\left(A^{-1} \boldsymbol{v}\right)\right)$ step by step.

1. By definition, we get

$$
\nabla_{\xi}\left(A^{-1} \boldsymbol{v}\right)=\left(\begin{array}{ll}
\left(c_{\theta}|\mathcal{F}| v_{1}-s_{\theta} v_{2}\right) \xi_{1} & \left(c_{\theta}|\mathcal{F}| v_{1}-s_{\theta} v_{2}\right) \xi_{2} \\
\left(s_{\theta}|\mathcal{F}| v_{1}+c_{\theta} v_{2}\right) \xi_{1} & \left(s_{\theta}|\mathcal{F}| v_{1}+c_{\theta} v_{2}\right) \xi_{2}
\end{array}\right)
$$

Since $\theta^{\prime}=\frac{1}{\sigma R}$ and $|\mathcal{F}|=1-\frac{\xi_{2}}{\sigma R}$, with $\sigma=\operatorname{sgn}(\theta)= \pm 1=\frac{1}{\sigma}$, we note that:

$$
\left(c_{\theta}\right)_{\xi_{1}}=-\frac{s_{\theta}}{\sigma R^{\prime}}, \quad\left(s_{\theta}\right)_{\xi_{1}}=\frac{c_{\theta}}{\sigma R^{\prime}}, \quad\left(c_{\theta}\right)_{\xi_{2}}=\left(s_{\theta}\right)_{\xi_{2}}=0, \quad|\mathcal{F}|_{\xi_{1}}=\frac{R^{\prime}}{R}(1-|\mathcal{F}|), \quad|\mathcal{F}|_{\xi_{2}}=\frac{|\mathcal{F}|-1}{\xi_{2}},
$$


which yields

$$
\left\{\begin{array}{l}
\left(\nabla_{\xi}\left(A^{-1} \boldsymbol{v}\right)\right)_{1,1}=-\frac{s_{\theta}}{\sigma R}|\mathcal{F}| v_{1}+c_{\theta} \frac{R^{\prime}}{R}(1-|\mathcal{F}|) v_{1}+c_{\theta}|\mathcal{F}|\left(v_{1}\right)_{\xi_{1}}-\frac{c_{\theta}}{\sigma R} v_{2}-s_{\theta}\left(v_{2}\right)_{\xi_{1}}, \\
\left(\nabla_{\xi}\left(A^{-1} \boldsymbol{v}\right)\right)_{1,2}=c_{\theta} \frac{|\mathcal{F}|-1}{\xi_{2}} v_{1}+c_{\theta}|\mathcal{F}|\left(v_{1}\right)_{\xi_{2}}-s_{\theta}\left(v_{2}\right)_{\xi_{2}}, \\
\left(\nabla_{\xi}\left(A^{-1} \boldsymbol{v}\right)\right)_{2,1}=\frac{c_{\theta}}{\sigma R}|\mathcal{F}| v_{1}+s_{\theta} \frac{R^{\prime}}{R}(1-|\mathcal{F}|) v_{1}+s_{\theta}|\mathcal{F}|\left(v_{1}\right)_{\xi_{1}}-\frac{s_{\theta}}{\sigma R} v_{2}+c_{\theta}\left(v_{2}\right)_{\xi_{1}}, \\
\left(\nabla_{\xi}\left(A^{-1} \boldsymbol{v}\right)\right)_{2,2}=s_{\theta} \frac{|\mathcal{F}|-1}{\xi_{2}} v_{1}+s_{\theta}|\mathcal{F}|\left(v_{1}\right)_{\xi_{2}}+c_{\theta}\left(v_{2}\right) \xi_{2} .
\end{array}\right.
$$

2. One can then compute both components of the vector $\boldsymbol{v} \cdot \nabla_{\xi}\left(A^{-1} \boldsymbol{v}\right)$ using the entries of the matrix $\nabla_{\xi}\left(A^{-1} \boldsymbol{v}\right)$ given above.

3. Finally, to find $A\left(\boldsymbol{v} \cdot \nabla_{\xi}\left(A^{-1} \boldsymbol{v}\right)\right)$, we remark that $c_{\theta}^{2}+s_{\theta}^{2}=1$, which allows many simplifications to occur in the expressions, and we get:

$$
\left\{\begin{array}{l}
\left(\mathrm{A}\left(\boldsymbol{v} \cdot \nabla_{\xi}\left(\mathrm{A}^{-1} \boldsymbol{v}\right)\right)\right)_{1}=v_{1}\left(v_{1}\right)_{\xi_{1}}+v_{2}\left(v_{1}\right)_{\xi_{2}}+\frac{v_{1}^{2}}{\mathrm{R}} \frac{\xi_{2} \sigma \mathrm{R}^{\prime}}{|\mathcal{F}| \mathrm{R}}-\frac{v_{1} v_{2}}{\mathrm{R}} \frac{2 \sigma}{|\mathfrak{F}|} \\
\left(\mathrm{A}\left(\boldsymbol{v} \cdot \nabla_{\xi}\left(\mathrm{A}^{-1} \boldsymbol{v}\right)\right)\right)_{2}=v_{1}\left(v_{2}\right)_{\xi_{1}}+v_{2}\left(v_{2}\right)_{\xi_{2}}+\frac{v_{1}^{2}}{\mathrm{R}} \sigma|\mathcal{F}|
\end{array}\right.
$$

Finally, we have obtained:

$$
A\left(\boldsymbol{v} \cdot \nabla_{\xi}\left(A^{-1} \boldsymbol{v}\right)\right)=\boldsymbol{v} \cdot \nabla_{\xi} \boldsymbol{v}+\Gamma(\boldsymbol{v}), \quad \text { where } \Gamma(\boldsymbol{v})=\left(\begin{array}{c}
\frac{v_{1}^{2}}{R\left(\xi_{1}\right)} \frac{\xi_{2} \sigma\left(\xi_{1}\right) R^{\prime}\left(\xi_{1}\right)}{|\mathcal{F}|\left(\xi_{1}, \xi_{2}\right) R\left(\xi_{1}\right)}-\frac{v_{1} v_{2}}{R\left(\xi_{1}\right)} \frac{2 \sigma\left(\xi_{1}\right)}{|\mathcal{F}|\left(\xi_{1}, \xi_{2}\right)} \\
\frac{v_{1}^{2}}{R\left(\xi_{1}\right)} \sigma\left(\xi_{1}\right)|\mathcal{F}|\left(\xi_{1}, \xi_{2}\right) .
\end{array}\right) .
$$

The additional term $\Gamma$ represents centripetal forces.

\section{Appendix B. Linear stability analysis of the four-equation model}

We perform a linear stability analysis of the four-equation model (5.22), in order to provide suitable expressions for the quantities $K_{1}$ and $K_{2}$. We linearize this system around the equilibrium state $W_{0}=\left(S_{0}, U_{0}, S \Psi_{0}, S \Pi_{0}\right)^{\top}$ such that $R\left(W_{0}\right)=0$, that is to say

$$
\mathrm{U}_{0}=\frac{\mathrm{Q}^{(0)}}{\mathrm{S}} \sqrt{\frac{\mathcal{J}}{\Lambda}} \neq 0, \quad S \Psi_{0}=S \Psi^{(0)} \frac{\mathcal{J}}{\Lambda} \quad \text { and } \quad S \Pi_{0}=S \Pi^{(0)} \frac{\mathcal{J}}{\Lambda} .
$$

Note that $W_{0}$ does not depend on $\Lambda$. For the sake of simplicity, let us temporarily assume that $Q^{(0)}, \Psi^{(0)}$ and $\Pi^{(0)}$ do not depend on $S$. This assumption, although false in the general case, allows us to greatly simplify the analysis and to set a framework for the S-dependent case.

To perform a linear stability analysis, we consider the eigenvalues $\left(\omega_{l}\right)_{1 \leqslant l \leqslant 4}$ of the matrix $M(k):=k A\left(W_{0}\right)+$ $i \nabla_{W} R(W)$, for all $k \in \mathbb{R}$. The system will be linearly stable if, for each $l \in \llbracket 1,4 \rrbracket, \operatorname{Im} \omega_{l} \leqslant 0$. For the four-equation 
model (5.22), the matrix $M(k)$ is given by:

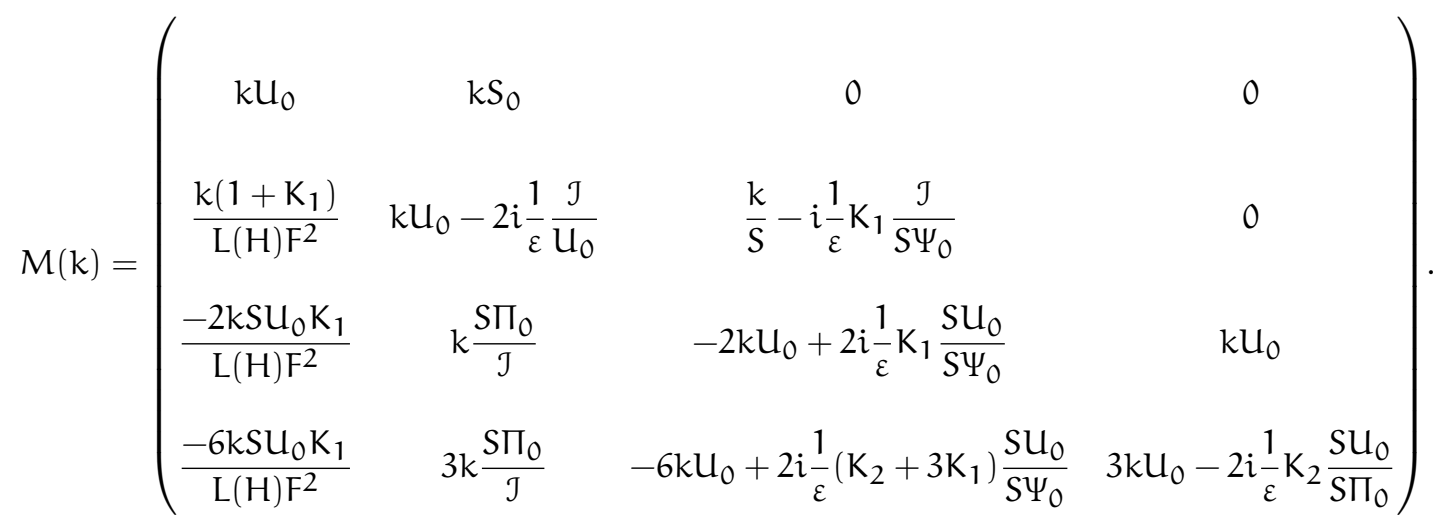

Let us start with the case $k=0$, where we can exactly compute the eigenvalues, to get the following expressions:

$$
\omega_{1}^{(0)}=0, \quad \omega_{2}^{(0)}=-2 i \frac{1}{\varepsilon} \frac{\mathcal{J}}{\mathrm{U}_{0}}, \quad \omega_{3}^{(0)}=2 i \frac{1}{\varepsilon} K_{1} \frac{S U_{0}}{S \Psi_{0}}, \quad \omega_{4}^{(0)}=-2 i \frac{1}{\varepsilon} K_{2} \frac{S U_{0}}{S \Pi_{0}} .
$$

The first two eigenvalues, $\omega_{1}^{(0)}$ and $\omega_{2}^{(0)}$, correspond to the ones of the classical shallow water system, and we get two additional ones related to the relaxation source terms. Since $\operatorname{sgn}\left(U_{0}\right)=\operatorname{sgn}\left(Q^{(0)}\right)=\operatorname{sgn}(\mathcal{J})$ in the usual range of applications, both shallow water eigenvalues have a non-positive imaginary part. Then, a natural simplification of the other two eigenvalues consists in taking $K_{1}$ and $K_{2}$ such that $\omega_{3}^{(0)}$ and $\omega_{4}^{(0)}$ become equal to $\omega_{2}^{(0)}$, as follows:

$$
\mathrm{K}_{1}=-\frac{S \Psi_{0}}{S U_{0}^{2}}=-\frac{S^{2} \Psi^{(0)}}{\left(Q^{(0)}\right)^{2}} \quad \text { and } \quad K_{2}=\frac{S \Pi_{0}}{S U_{0}^{2}}=\frac{S^{2} \Pi^{(0)}}{\left(Q^{(0)}\right)^{2}}
$$

With this choice, we immediately obtain that all four eigenvalues (B.1) have a non-positive imaginary part.

However, this case $k=0$ only yields necessary conditions for the linear stability, and we need to take care of the case of a nonzero $k$. After tedious computations, we get two eigenvalues $\omega_{l}=\frac{1}{\varepsilon} \sum_{j=0}^{3} \omega_{l}^{(j)}$, for $l \in\{1,2\}$, such that $\chi\left(k, \omega_{l}\right)=\mathcal{O}\left((\varepsilon)^{3}\right)$, where $\omega \mapsto \chi(k, \omega)$ is the characteristic polynomial of $M(k)$. For the sake of conciseness, we do not write their expressions here. These eigenvalues have a negative imaginary part (i.e. the system is asymptotically linearly stable) as soon as the following condition is satisfied:

$$
\mathrm{u}_{0}^{2}<\frac{4 \mathrm{~S}_{0}}{\mathrm{~L}(\mathrm{H})}+\Pi
$$

This condition can be compared to the asymptotic linear stability condition for the shallow water equations, which reads $\mathrm{U}_{0}^{2}<4 \mathrm{~S}_{0} / \mathrm{L}(\mathrm{H})$. Note that, since $\Pi \geqslant 0$, the linear stability condition for the four-equation model is less restrictive than that of the shallow water equations.

Lastly, recall that we had assumed that $\mathrm{U}^{(0)}, \Psi^{(0)}$ and $\Pi^{(0)}$ did not depend on S. We now address the real case where these quantities depend on $S$. To shorten the notations, let us introduce $\mathfrak{c}(S)$ such that $\mathcal{J}=\mathrm{U}|\mathrm{U}| /(\mathrm{S} \mathfrak{c})$, $\Psi_{S}^{(0)}=\partial\left(S \Psi^{(0)}\right) / \partial S$ and $\Pi_{S}^{(0)}=\partial\left(S \Pi^{(0)}\right) / \partial S$. Under the condition (B.3), we obtain the following additional sufficient condition on $S$ for the asymptotic linear stability of the four-equation model:

$$
\mathfrak{c}^{2}\left(3 \Psi_{\mathrm{S}}^{(0)}-2 \Pi_{\mathrm{S}}^{(0)}\right)+\mathrm{S}^{2}\left(\mathrm{U}^{(0)}\right)^{2} \mathfrak{c}_{\mathrm{S}}^{2}+\left(2 \mathrm{~S}\left(\mathrm{U}^{(0)}\right)^{2}-\Pi^{(0)}+S \Psi_{\mathrm{S}}^{(0)}\right) \mathfrak{c}_{\mathrm{S}}<0
$$

\section{References}

[1] M. Amara, D. Capatina-Papaghiuc, and D. Trujillo. Hydrodynamical modelling and multidimensional approximation of estuarian river flows. Comput. Visual. Sci., 6(2-3):39-46, 2004. 
[2] P. D. Bates. Computational Fluid Dynamics. Wiley-Blackwell, 2005.

[3] G. Bispen, K. R. Arun, M. Lukáčová-Medvid'ová, and S. Noelle. IMEX large time step finite volume methods for low Froude number shallow water flows. Commun. Comput. Phys., 16(2):307-347, 2014.

[4] D. Bresch and P. Noble. Mathematical Justification of a Shallow Water Model. Methods Appl. Anal., 14(2):87$118,2007$.

[5] D. Chen and J.G. Duan. Modeling width adjustment in meandering channels. J. Hydrol., 321(1-4):59-76, 2006.

[6] V. T. Chow. Open-channel hydraulics. McGraw-Hill civil engineering series. McGraw-Hill, 1959.

[7] G. Coriolis. Sur l'établissement de la formule qui donne la figure des remous, et sur la correction qu'on doit y introduire pour tenir compte des différences de vitesse dans les divers points d'une même section d'un courant. Ann. des Ponts et Chaussées, 11(1):314-335, 1836.

[8] F. Couderc, R. Madec, J. Monnier, and J.-P. Vila. DassFlow v2.00.00 : User and Developer Guide. CNRS / IMT / INSA Toulouse / UPS, http://www.math.univ-toulouse.fr/DassFlow, 2015.

[9] D. C. Dammuller, S. Murty Bhallamudi, and M. Hanif Chaudhry. Modeling of Unsteady Flow in Curved Channel. J. Hydraul. Eng., 115(11):1479-1495, 1989.

[10] A. Decoene, L. Bonaventura, E. Miglio, and F. Saleri. Asymptotic derivation of the section-averaged shallow water equations for natural river hydraulics. Math. Models Methods Appl. Sci., 19(03):387-417, 2009.

[11] F. Engelund. Instability of flow in a curved alluvial channel. J. Fluid Mech., 72:145-160, 1975.

[12] E. D. Fernández-Nieto, J. Marin, and J. Monnier. Coupling superposed 1D and 2D shallow-water models: Source terms in finite volume schemes. Comput. \& Fluids, 39(6):1070-1082, 2010.

[13] W. H. Finlay, J. B. Keller, and J. H. Ferziger. Instability and transition in curved channel flow. J. Fluid Mech., 194:417-456, 1988.

[14] S. Gavrilyuk and H. Gouin. Geometric evolution of the Reynolds stress tensor. Intl J. Engng Sci., 59:65-73, 2012.

[15] J.-M. Hervouet. Hydrodynamics of Free Surface Flows. John Wiley \& Sons, Ltd, 2007.

[16] Ikeda, S. and McEwan, I. K. (Eds.) Flow and sediment transport in compound channels. London: CRC Press, 2009.

[17] K. A. Ivanova and S. L. Gavrilyuk. Structure of the hydraulic jump in convergent radial flows. J. Fluid Mech., 860:441-464, 2018.

[18] O. E. Jensen. The thin liquid lining of a weakly curved cylindrical tube. J. Fluid Mech., 331:373-403, 1997.

[19] R. J. LeVeque. Finite Difference Methods for Ordinary and Partial Differential Equations: Steady-State and Timedependent Problems (Classics in Applied Mathematics). Society for Industrial and Applied Mathematics, 2007.

[20] M. J. Lighthill and G. B. Whitham. On Kinematic Waves. I. Flood Movement in Long Rivers. Proceedings of the Royal Society A: Mathematical, Physical and Engineering Sciences, 229(1178):281-316, 1955. 
[21] P. Luchini and F. Charru. Consistent section-averaged equations of quasi-one-dimensional laminar flow. J. Fluid Mech., 656:337-341, 2010.

[22] J. H. Masliyah. On laminar flow in curved semicircular ducts. J. Fluid Mech., 99:469-479, 1980.

[23] J. E. Miller. Basic concepts of kinematic-wave models. U.S. Geological Survey, 1984.

[24] J. Monnier, F. Couderc, D. Dartus, K. Larnier, R. Madec, and J.-P. Vila. Inverse algorithms for 2D shallow water equations in presence of wet dry fronts: Application to flood plain dynamics. Adv. Water Resour., 97:11-24, 2016.

[25] L. F. Moody. Friction Factors for Pipe Flow. Transactions of the American Society of Mechanical Engineers, 66:671-681, 1944.

[26] A. J. Odgaard. River-Meander Model. I: Development. J. Hydraul. Eng., 115(11):1433-1450, 1989.

[27] A. J. Odgaard. River-Meander Model. II: Applications. J. Hydraul. Eng., 115(11):1451-1464, 1989.

[28] G. L. Richard, A. Duran, and B. Fabrèges. A new model of shoaling and breaking waves. Part 2. Run-up and two-dimensional waves. J. Fluid Mech., 867:146-194, 2019.

[29] G. L. Richard and S. L. Gavrilyuk. A new model of roll waves: comparison with Brock's experiments. J. Fluid Mech., 698:374-405, 2012.

[30] G. L. Richard, M. Gisclon, C. Ruyer-Quil, and J. P. Vila. Optimization of consistent two-equation models for thin film flows. Eur. J. Mech. B. Fluids, 76:7-25, 2019.

[31] G. L. Richard, A. Rambaud, and J.-P. Vila. Consistent equations for open-channel flows in the smooth turbulent regime with shearing effects. J. Fluid Mech., 831:289-329, 2017.

[32] G. L. Richard, C. Ruyer-Quil, and J.-P. Vila. A three-equation model for thin films down an inclined plane. J. Fluid Mech., 804:162-200, 2016.

[33] A. Shi, M. H. Teng, and T. Y. Wu. Propagation of solitary waves through significantly curved shallow water channels. J. Fluid Mech., 362:157-176, 1998.

[34] N. S. Sivakumaran, T. Tingsanchali, and R. J. Hosking. Steady shallow flow over curved beds. J. Fluid Mech., 128:469-487, 1983.

[35] B. Snyder, J. R. Hammersley, and D. E. Olson. The axial skew of flow in curved pipes. J. Fluid Mech., 161:281294, 1985.

[36] J. J. Stoker. Water Waves. Wiley-Interscience, 1992.

[37] V. M. Teshukov. Gas-dynamic analogy for vortex free-boundary flows. J. Appl. Mech. Tech. Phys., 48(3):303309, 2007.

[38] J. P. Vila. Modélisation mathématique et simulation numérique d'écoulements à surface libre. Houille Blanche, (6-7):485-490, 1984.

[39] C. B. Vreugdenhil. Numerical Methods for Shallow-Water Flow. Springer Netherlands, 1994.

[40] H. J. De Vriend. Velocity redistribution in curved rectangular channels. J. Fluid Mech., 107:423-439, 1981. 
[41] N. A. Zaghloul. Hydraulic exponents M and N for gravity flow pipes. Adv. Water Resour., 21(3):185-191, 1998.

[42] A. R. Zarrati, Y. C. Jin, and S. Karimpour. Semianalytical model for shear stress distribution in simple and compound open channels. J. Hydraul. Eng., 134(2):205-215, 2008. 